\section{Check for updates}

Cite this: Chem. Soc. Rev., 2020, 49, 5601

Received 31st March 2020

DOI: $10.1039 / c 9 c s 00594 c$

rsc.li/chem-soc-rev

\title{
Electrical conductivity and magnetic bistability in metal-organic frameworks and coordination polymers: charge transport and spin crossover at the nanoscale
}

\section{Introduction}

${ }^{a}$ Instituto de Ciencia Molecular, Universitat de València, Catedrático José Beltrán 2, 46980 Paterna, Spain. E-mail: carlos.marti@uv.es

${ }^{b}$ Centre for Membrane Separations, Adsorption, Catalysis, and Spectroscopy for Sustainable Solutions (cMACS), Department of Microbial and Molecular Systems, KU Leuven, Celestijnenlaan 200F, Leuven 3001, Belgium

Electronic devices shape the world as we know it. The tremendous technological advances that we experienced in the last 50 years relied on the progressive miniaturization of these devices and their components to construct increasingly complex and

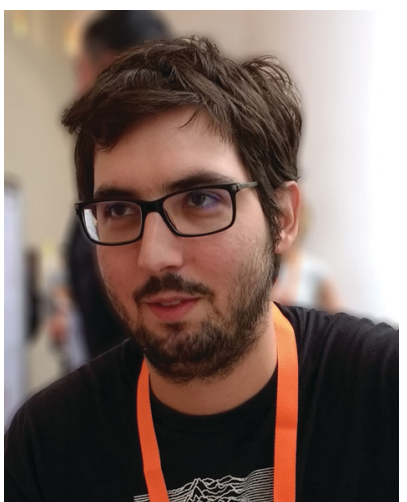

Víctor Rubio-Giménez
Victor Rubio-Giménez obtained his $\mathrm{PhD}$ in Nanoscience and Nanotechnology at the ICMol (Universitat de València) in 2019. Under the supervision of Prof. Eugenio Coronado and Prof. Carlos MartiGastaldo, he prepared ultrathin films of MOFs and CPs and studied their electronic and magnetic properties at the nanoscale. Subsequently, he joined Prof. Rob Ameloot's group at $K U$ Leuven after receiving a Marie Skłodowska-Curie Actions - Seal of Excellence Postdoctoral Fellowship from the Research Foundation Flanders (FWO) to work in the vapour deposition of MOF nanofilms for memory devices.

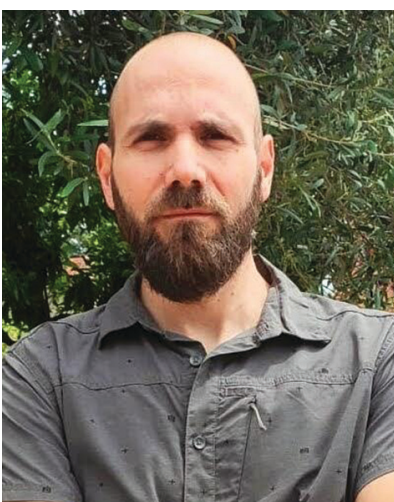

Sergio Tatay
Sergio Tatay completed his $P h D$ in Chemistry at the ICMol (Universitat de València) on the design of bistable metallic complexes in 2008. In 2009, he joined the Unité Mixte of Physique CNRS/ THALES (UMR137) as a Marie Curie Fellow. There, he worked in the integration of organic materials into spintronic devices. At the beginning of 2013, he returned to the ICMol as a Juan de la Cierva-MC CIG Fellow. In 2017 he received a Ramón y Cajal

Fellowship. His current research is focused on the development of electronic devices based on conductive MOFs for electrocatalysis or energy storage applications. 
powerful platforms. This achievement has been possible thanks to low-cost production through scalable complementary metal-oxide-semiconductor (CMOS) microfabrication. However, traditional CMOS materials (metal chalcogenides) suffer from a fundamental design limitation due to the scarcity of usable inorganic anions. As a consequence, the electronics field is on the verge of a dramatic transition from a materials point of view. As suggested by the International Roadmap for Devices and Systems (IRDS), ${ }^{1,2}$ the industry should move on from inorganic and silicon-based materials towards organic and hybrid organic-inorganic "beyond CMOS" materials that can improve existing properties and bring additional ones to the table. Presently, materials scientists are shifting from purely inorganic, organic and silicon-based materials towards hybrid organic-inorganic materials to construct increasingly complex and powerful electronic devices. In this context, it is undeniable that coordination polymers (CPs) and metal-organic frameworks (MOFs) are carving a niche for themselves in the electronics world amongst these "beyond CMOS" materials.

CPs are hybrid organic-inorganic systems that crystallize by the assembly of metal ions and polytopic organic ligands to form $2 \mathrm{D}$ or $3 \mathrm{D}$ extended structures. Compared to classical CMOS materials used in electronic devices, CPs combine the extended structure and high crystallinity of inorganic solids with the synthetic versatility (structural and electronic manipulation through chemical modification of ligands and metal clusters), ${ }^{3,4}$ easy processability and mechanical flexibility ${ }^{5,6}$ of organic materials. Furthermore, in some cases the structural disposition of metallic nodes and organic ligands creates voids or pores that can be occupied by gases or other guests. First reported as such by Robson and co-workers, ${ }^{7}$ this particular class of CPs denoted as MOFs ${ }^{8,9}$ or porous coordination polymers (PCPs) ${ }^{10}$ offer the possibility of indirectly manipulating their properties and even introduce extra functionalities simply

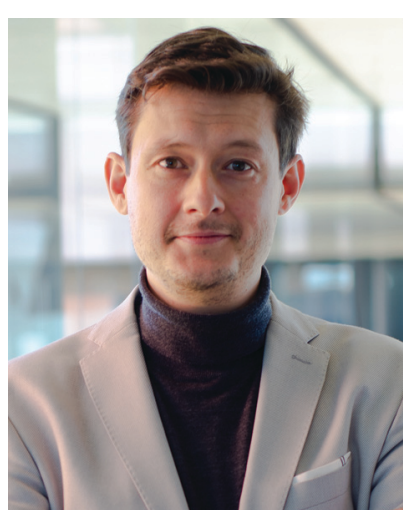

Carlos Martí-Gastaldo
Carlos Marti-Gastaldo obtained his PhD in Chemistry from the Universitat de València in 2009. He then moved to the University of Liverpool, first as a Marie Curie Postdoctoral Fellow and next as University Research Fellow of the Royal Society. In 2014, he moved back to the ICMol with a Ramón y Cajal Fellowship to lead one of the strategic research lines of the Maria de Maeztu Excellence programme. In 2016, he received an ERC Starting grant and established a new research group at the centre focused on the design of functional inorganic materials (FuniMAT). His current research interests encompass the design and processing of inorganic porous materials for biological and environmental-related applications. by infiltration with active guests. ${ }^{11}$ Nevertheless, most consolidated applications, employ MOFs as innocent non-active frameworks to store ${ }^{12-17}$ or separate gases and other guests. ${ }^{18,19}$ Later, the possibility to exploit pore chemistry emerged with the use of MOFs heterogeneous as catalysts. ${ }^{20-22}$ Still, all the above applications are focused on the physical and chemical properties of the internal pores of MOFs while the rest of the framework remains in a secondary role. When MOFs and other CPs become fundamental parts of electronic devices an opposite situation takes place, the framework plays a central role in device performance, manipulating the electrons that come into contact with it. The way in which these systems interact with electrons is fundamentally manifested through their electronic properties, i.e. magnetism and electrical conductivity. Coincidently, MOFs and CPs offer a very competitive advantage to tune these properties by chemical design thanks to their molecular nature. Chemists have the capability of easily producing tailor-made materials with an almost infinite palette of ligands and metal ion combinations. Furthermore, the connectivity in between them can also be altered, and in the case of MOFs there is the option of incorporating functional guests into the pores. Nevertheless, material design and bulk synthesis is only the first step in the device integration course. The subsequent one being processing these active materials into nanometric thin films to be integrated in the final electronic devices.

In this review, we re-examine the different approaches to render MOFs and CPs electrically conductive, as well as the strategies to nanostructure and process them as thin films to act as active interfaces in electronic devices. In this sense, various reviews have recently highlighted their potential ${ }^{23-28}$ centring around the electronic properties of MOFs and CPs. However, most of them miss out on a second relevant property: magnetism, and mostly on the interplay between both electronic and magnetic properties of these materials. Regarding magnetic properties, we center on magnetic bistability as mainly represented by spin crossover (SCO), the magnetic property that has received the most attention from a device point of view for MOFs and CPs. These bistable materials have great potential for the development of functional nanodevices, ${ }^{29}$ provided that the critical effects of nanostructuration (particle size reduction, crystallinity, interparticle organization, surface defects, etc.) on the spin transition are well understood along with the electronic properties of the respective spin states. In other words, comprehending how the spin state may impact the electrical response of the SCO material in a device setting.

Finally, we examine a selection of the most prominent examples of working devices based on MOFs and CPs, which in our eyes exemplify the potential perspectives of device integration for these materials. The search for novel crystalline polymeric materials and their nanostructuration into electronic devices is a challenging task ahead of chemists, physicists and chemical engineers. This review aims to reassess the steps taken so far towards integrating CPs and MOFs as active components in electronic and bistable devices. To the best of our knowledge, no other published review highlights the 
importance of processing MOFs and CPs as high-quality ultrathin films together with the careful examination of their electronic and magnetic properties at the nanoscale, the ultimate target being the fabrication of functional nanodevices.

\section{Electrical conductivity in framework materials}

This property measures the ability of a material to conduct electrical current, that is, the ease with which charge carriers (electrons or holes) can travel across it. The magnitude experimentally measured is conductivity $\left(\sigma\right.$; units: $\mathrm{S} \mathrm{cm}^{-1}$ or $\Omega^{-1} \mathrm{~cm}^{-1}$ ) which is the inverse of resistivity $(\rho)$, an intrinsic property of materials calculated by taking into account the geometrical dimensions of the sample (length between the contacts and cross-sectional area) and its electrical resistance $(R)$ at a given voltage $(V)$. In principle, the highly ordered crystal structure of MOFs or CPs warrants the application of band theory to rationalize their electrical conductivity and electronic structure. According to it, the discrete energy levels that an electron can occupy in an ordered solid merge to form continuous energy bands. At absolute zero, the filled bands are referred to as the valence band $(\mathrm{VB})$, while the unoccupied band with the lowest energy is the conduction band (CB). ${ }^{30}$ Thus, solids can be generally classified as metals, semiconductors and insulators (Fig. 1) owing to their respective electronic band structures and the separation between the maximum of the VB and the $\mathrm{CB}$ minimum, defined as the bandgap $\left(E_{\mathrm{g}}\right)$. In metallic conductors the $\mathrm{CB}$ and VB overlap $\left(E_{\mathrm{g}}=0\right)$, the Fermi level $\left(E_{\mathrm{F}}\right)$ crosses a partially filled band and all the electrons are free charge carriers. Owing to its high charge density the electrical conductivity is in turn very high. For semiconductors and insulators there is an energy gap, which is small for semiconductors $\left(0<E_{\mathrm{g}}<3 \mathrm{eV}\right)$ and large for insulators $\left(E_{\mathrm{g}}>3 \mathrm{eV}\right)$. However, the boundary between the two is sometimes blurry as large bandgap semiconductors are considered insulators or vice versa, then additional information about the conduction mechanism has to be gathered as discussed below. In these cases, the Fermi level is located in the energy gap between the VB and the CB, either in the middle (intrinsic semiconductors), closer to the VB (p-type semiconductor) or closer to the $\mathrm{CB}$ (n-type semiconductor). The energy difference between the Fermi level and the VB maximum or the $\mathrm{CB}$ maximum is the activation energy $\left(E_{\mathrm{a}}\right)$. At finite temperature, VB electrons can be thermally promoted into the
$\mathrm{CB}$, leaving holes in the VB. Then both electrons and holes become free charges. Therefore, the conductivity increases with temperature in the case of semiconductors. Solids with different band structures will yield different electrical conductivity values depending also on the mobility of electrons and holes, which reflects the efficiency of the charge transport.

Charge carriers in a crystal are not completely free to move, but instead interact with the periodic potential of the lattice. Poorer orbital overlaps will lead to higher interactions and lower mobility. Moreover, a high density of charge scattering sites, such as disorder, defects, impurities or grain boundaries will further reduce carrier mobility to the point that charge carriers will become localized at specific sites with discrete energy levels, and charge transport will be possible only when charge carries hop between neighbouring sites with hopping probability scaling with temperature. However, the magnitude of this dependence can be different to that expected for band transport regime. In general, band transport yields higher charge mobility and higher conductivity than hopping. From a design perspective, both modes of transport require good spatial and energetic overlap between orbitals of appropriate symmetry.

Despite being fundamental for their device integration, the ability to transport charge carriers through 2D or 3D CPs and MOFs has not been studied as in-depth as for their purely organic counterparts, ${ }^{31-34}$ especially if we include graphene ${ }^{35-39}$ and other carbon nanoforms ${ }^{40-43}$ in the latter group. In fact, the popularity of these materials in electronics does not compare with the explosion of low dimensional materials during the last decade. Traditionally, conductivity has not been a targeted property in CPs (except in 1D metal-organic wires) ${ }^{44}$ and particularly in MOFs, so most of them are composed of hard metal ions and redox innocent organic ligands which does not favour charge carrier mobility. Thus, most MOFs and CPs qualify as insulators, due to their low conductivity at room temperature. However, in the last decade this situation has started to change with the appearance of various strategies to produce highly conductive CPs ${ }^{27,44-46}$ and especially MOFs. ${ }^{26,47-56}$ This has allowed researchers to envision the future integration of these materials as active elements in functional electronic devices. $^{24,25,57,58}$ Next, a brief description and classification of the different mechanistic approaches for conductive MOFs and CPs is presented. This section is directed towards familiarizing the reader with the materials and approaches for the subsequent discussion on nanostructuration strategies, combination with magnetic bistability and serves to highlight the relative immaturity of the field. For more comprehensive assessments of electrical
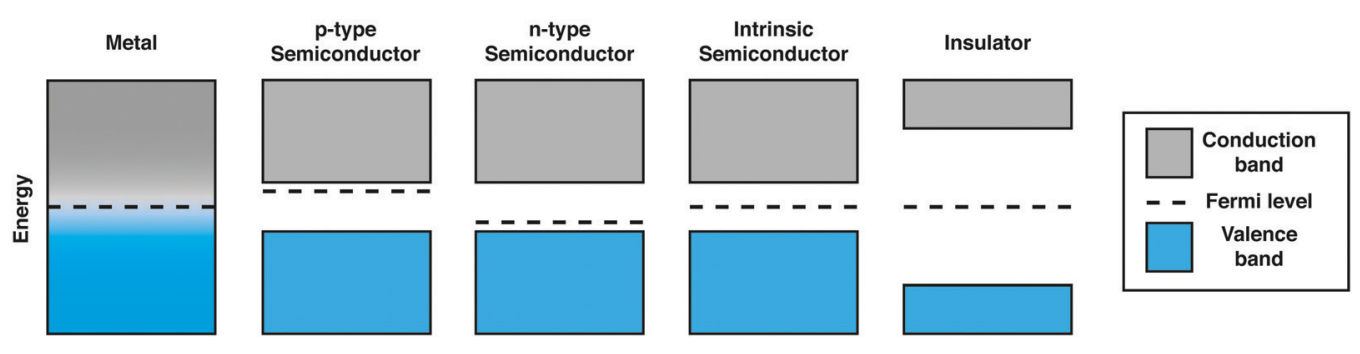

Fig. 1 Schematic band structure representations of the different types of solids. 
conductivity in framework materials, we direct the reader to the recent specific reviews on the matter. ${ }^{52-55}$

\subsection{Guest-induced conductivity}

The guest-induced conductivity approach can obviously be used only with porous materials. In this tactic, an insulating network is turned conductive thanks to an adsorbed guest molecule, organic polymer or metallic nanoparticle (Fig. 2). The obvious downside of this strategy is that a fraction of the MOF's porosity

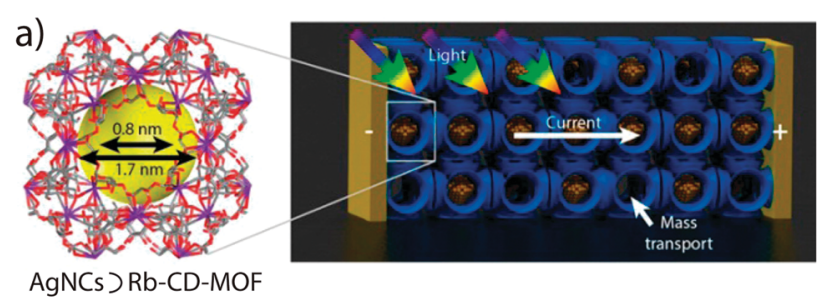

AgNCs ) Rb-CD-MOF

b)

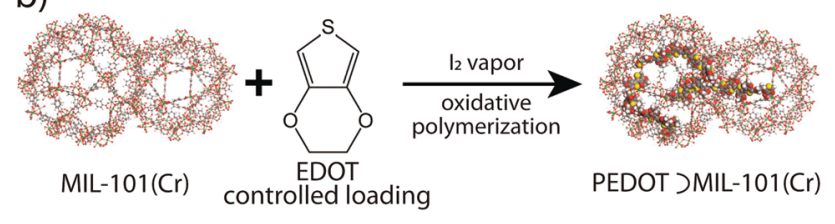

c)

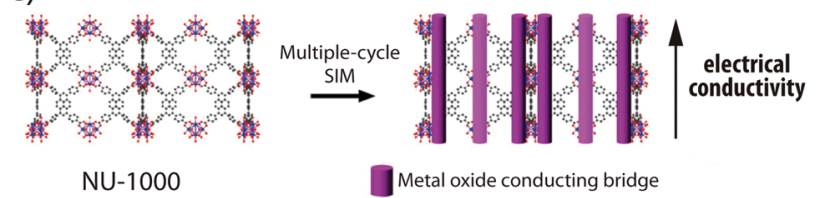

d)
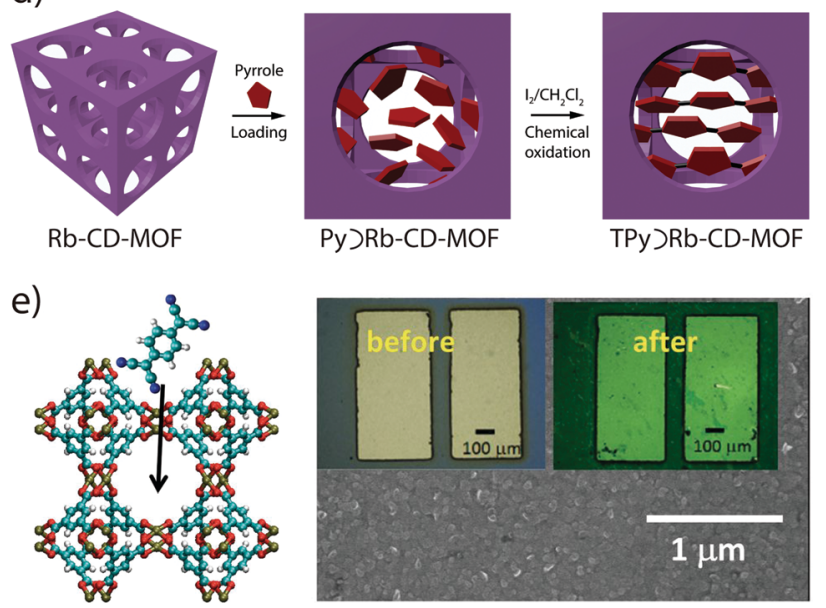

Fig. 2 Different variants of the guest-induced conductivity strategy. (a) Infiltration with metallic NCs (Ag-NCs). (b) Generation of a conductive polymer in the MOF pores. (c) Generation of conductive metal oxide strands. (d) Generation of charge transfer salts templated by the framework host-guest chemistry. (e) Infiltration with a redox-active, conjugated guest molecule (TCNQ). (a) Reproduced from ref. 59 with permission from the American Chemical Society, copyright 2015. (b) Reproduced from ref. 63 with permission from the American Chemical Society, copyright 2016. (c) Reproduced from ref. 60 with permission from the American Chemical Society, copyright 2018. (d) Reproduced from ref. 65 with permission from Wiley, copyright 2019. (e) Reproduced from ref. 80 with permission from the American Association for the Advancement of Science, copyright 2014. is sacrificed at the cost of electrical conductivity. In some cases, the guest is itself conductive, and simply provides a viable channel for charge transport to go through, while in others the guest induces, either a redox transformation (leading to the generation of charge carriers within the framework) or structural transformations resulting in new conductive pathways that make the previously insulating framework conductive.

2.1.1. Infiltration of conductive guests. When metal nanoparticles (NPs), nanoclusters (NC) or conductive organic polymers are infiltrated into a MOF, they do not interact with the framework to create additional conductive channels but act as conductive channels of their own. As visible in Fig. 2a, Grzybowski et al. infiltrated Rb-CD-MOF with $\mathrm{AgNO}_{3}$ which spontaneously reduced in the pores to form $\mathrm{Ag}$ nanoclusters (Ag-NCs). ${ }^{59}$ Charge transport $\left(\sigma=2.15 \times 10^{-7} \mathrm{~S} \mathrm{~cm}^{-1}\right.$ upon light irradiation) then occurred via tunnelling between the spatially separated Ag-NCs that did not entirely fill the pores, thus the MOF was still porous afterwards (BET $>600 \mathrm{~m}^{2} \mathrm{~g}^{-1}$ ). This light-assisted tunnelling conductivity was reproduced in NCloaded MIL-53 crystals as well. ${ }^{59} \mathrm{~A}$ more sophisticated methodology was recently used by Farha, Hupp and collaborators who grew continuous strands tetratin(Iv)oxy clusters along the pore channels of NU-1000 (Fig. 2b). ${ }^{60}$ However, the final material displayed similar conductivity $\left(\sigma=1.8 \times 10^{-7} \mathrm{~S} \mathrm{~cm}^{-1}\right)$ and is less porous (BET $=650 \mathrm{~m}^{2} \mathrm{~g}^{-1}$ ) than the $\mathrm{NiCB} \supset \mathrm{NU}-1000$ system (see below). ${ }^{61}$ Lastly, in the category of conductive polymers as guests, there are examples with polypyrrole (PPy), polyethylenedioxythiophene (PEDOT) and polythiophene (PT). Similar conductivities were achieved in PPy $\supset\left[\mathrm{Cd}(\mathrm{NDC})_{0.5}(\mathrm{PCA})\right]^{62}(\sigma=$ $1 \times 10^{-3} \mathrm{~S} \mathrm{~cm}^{-1} ; \mathrm{H}_{2} \mathrm{NDC}=2$,6-napthalenedicarboxylic acid; HPCA: 4-pyridinecarboxylic acid) and PEDOT $\supset$ Cr-MIL-101 $(\sigma=$ $1.1 \times 10^{-3} \mathrm{~S} \mathrm{~cm}^{-1}$, Fig. $2 \mathrm{c}$ ),${ }^{63}$ but only the latter maintained a high porosity $\left(\mathrm{BET}=803 \mathrm{~m}^{2} \mathrm{~g}^{-1}\right)$. For $\mathrm{PT} \supset \mathrm{NU}-1000$ the retained porosity was much larger $\left(\right.$ BET $\left.=1560 \mathrm{~m}^{2} \mathrm{~g}^{-1}\right)$ but the conductivity was again relatively low $\left(\sigma=1.3 \times 10^{-7} \mathrm{~S} \mathrm{~cm}^{-1}\right)$ for this particular Zr-MOF system. ${ }^{64}$ Marti-Gastaldo and co-workers also demonstrated the possibility of restricting the growth of PPy by confined oxidation of pyrrole (Py) monomers after encapsulation in $\mathrm{Rb}-\mathrm{CD}-\mathrm{MOF}$ to generate terpyrrole (TPy) charge transfer salts (Fig. 2d). ${ }^{65}$ Whereas Rb-CD-MOF and Py $\supset$ Rb-CD-MOF behave as insulators $\left(\sigma=10^{-11}-10^{-12} \mathrm{~S} \mathrm{~cm}^{-1}\right)$, TPy $\supset \mathrm{Rb}-\mathrm{CD}-\mathrm{MOF}$ displays a million-fold enhancement of the electrical conductivity up to $5 \times 10^{-6} \mathrm{~S} \mathrm{~cm}^{-1}$. More recently, Ballav and co-workers polymerized PEDOT and PPy inside the pores of UiO-66. Hence, boosting its conductivity up to $2 \times 10^{-2} \mathrm{~S} \mathrm{~cm}^{-1}$ for PPy $\supset$ UiO-66 and $10^{-3} \mathrm{~S} \mathrm{~cm}^{-1}$ for PEDOT $\supset$ UiO-66. Moreover, thanks to the polymerization mainly taking place in one of the pores of UiO-66, the infiltrated MOFs also kept 69\% (PPy $\supset$ UiO-66) and $57 \%$ (PEDOT $\supset$ UiO-66) of the original BET values. In all three cases, the MOF was first infiltrated with oligomers that were later polymerized inside the pores. ${ }^{66}$

2.1.2. Infiltration of non-conductive guests. The first example of this strategy was reported by Zeng et al. in 2010, the insulating $\left[\mathrm{Zn}_{3}(\mathrm{DL}-\mathrm{lac})_{2}(\mathrm{pybz})_{2}\right](\mathrm{DL}-\mathrm{lac}=$ lactate; pybz $=4$-pyridylbenzoate $)$ was turned semiconductive $\left(\sigma=3.4 \times 10^{-3} \mathrm{~S} \mathrm{~cm}^{-1}\right)$ by loading the pores with $\mathrm{I}_{2}{ }^{67}$ Afterwards, the $\mathrm{I}_{2}$-doping strategy has been successfully 
reproduced with a considerable number of insulating MOFs. The appearance of electrical conductivity was due to either donoracceptor interactions between $I_{2}$ and ligand $\pi$ electrons, ${ }^{68-72}$ the oxidation of the network ${ }^{73-75}$ or conduction through polyiodide ions. ${ }^{76-78}$ Although in some of these conduction mechanisms were not clearly elucidated. It is worth mentioning the particular case of $\left\{\left[\mathrm{Cu}_{6}(\mathrm{pybz})_{8}(\mathrm{OH})_{2}\right] \cdot \mathrm{I}_{5}{ }^{-} \cdot \mathrm{I}_{7}{ }^{-}\right\}_{n}$, which was directly synthesized with an array of polyiodide ions inside the framework. ${ }^{79}$ Another variation of this strategy to tune electrical conductivity in MOFs is to infiltrate the nanopores with redox-active, conjugated guest molecules. Allendorf and co-workers demonstrated this by using thin-film devices of the MOF $\mathrm{Cu}_{3}(\mathrm{BTC})_{2}$ (also known as HKUST-1; BTC = benzene-1,3,5-tricarboxylic acid) infiltrated with 7,7,8,8-tetracyanoquinododimethane (TCNQ).$^{80}$ An increase of over six orders of magnitude in electrical conductivity was achieved (Fig. 2e), reaching values as high as $7 \times 10^{-2} \mathrm{~S} \mathrm{~cm}^{-1}$, tuneable by changing the exposure time to TCNQ solutions. Moreover, in this case, the MOF maintained a moderate porosity $\left(\right.$ BET $\left.=214 \mathrm{~m}^{2} \mathrm{~g}^{-1}\right)$ after guest infiltration. Spectroscopic data and first-principles modelling suggested that the conductivity arose from TCNQ guest molecules bridging the binuclear copper paddlewheels in the framework, leading to strong electronic coupling between the dimeric $\mathrm{Cu}$ subunits. $^{80}$ Later, Wöll and collaborators further clarified the conductivity mechanism with an extended hopping transport model in which carriers hop from guest to guest mediated by virtual occupancy of a MOF site. ${ }^{81}$ This TCNQ-induced conductivity was successfully replicated by Loh et al. with a 2D Cu-porphyrin MOF. ${ }^{82}$ Likewise, ferrocene has also been used as redox-active guest in HKUST-1 by Wöll and collaborators. ${ }^{83,84}$ However, significantly lower conductivity values $\left(\sigma=2 \times 10^{-9} \mathrm{~S} \mathrm{~cm}^{-1}\right)$ were obtained due to the larger size of ferrocene compared to TCNQ, which implies a lower guest loading in the pores. Finally, cationic methyl viologen, ${ }^{85}$ metallacarboranes ${ }^{61}$ and fullerene $\left(\mathrm{C}_{60}\right)^{86,87}$ have also been incorporated to MOFs to achieve conductivity via donor-acceptor interactions with the framework. Remarkably, in the second case, nickel(Iv) bis(dicarbollide) was infiltrated in the micropores of NU-1000 leaving the larger mesopores free $\left(B E T=1260 \mathrm{~m}^{2} \mathrm{~g}^{-1}\right.$ after infiltration), although the measured conductivity was rather moderate $\left(\sigma=2.7 \times 10^{-7} \mathrm{~S} \mathrm{~cm}^{-1}\right) .{ }^{61}$ Nevertheless, for the latter case, $\mathrm{C}_{60} \supset$ NU-901 exhibited even higher porosity $\left(\mathrm{BET}=1550 \mathrm{~m}^{2} \mathrm{~g}^{-1}\right.$ after infiltration) and conductivity $\left(\sigma=1 \times 10^{-3} \mathrm{~S} \mathrm{~cm}^{-1}\right)$ values. $^{86}$

\subsection{Intrinsic conductivity through the framework: through- space and through-bond approaches}

As mentioned above, the other pathway to achieve MOF conductivity is to design intrinsically conductive frameworks. This can be more challenging from a synthetic point of view but has several advantages towards the guest-doping approach: (i) The porosity is obviously preserved, (ii) there is no need for postsynthetic modifications, which can have a negative effect on crystallinity, and most importantly (iii) conductivity values are higher on average. In the intrinsically conductive MOFs and CPs group we can distinguish two different mechanisms: through-space and through-bond conduction.

2.2.1. Through-space conductivity. In the through-space approach, $\pi-\pi$ stacking interactions between electroactive moieties in the framework offer conduction paths responsible for charge transport. The most widespread option to produce this type of conductive MOFs is the use of tetrathiafulvalene (TTF) derivatives as ligands, based on this molecule's huge popularity to prepare charge transfer salts and organic conductors. ${ }^{8-91}$ Although examples of 1D metallic chains had appeared earlier, ${ }^{44,92}$ the first examples of conductive TTF-based 2D or 3D CPs are nonporous and date back to 2002 with the report of a $\mathrm{Ag}^{\mathrm{I}} 3 \mathrm{D}$ CP with an S-donor TTF ligand. ${ }^{93}$ After another example of a conductive $\mathrm{Ag}^{\mathrm{I}}$ 3D with S-donor TTF ligands by Wang and co-workers in $2008,{ }^{94}$ the first family of CPs with carboxylate-TTFs was reported by Férey and collaborators. Among them, MIL-135(K) presented the highest conductivity known to date for a TTF-based CP $\left(\sigma=1 \times 10^{-3} \mathrm{~S} \mathrm{~cm}^{-1}\right) .{ }^{95}$ Then, Dincă's group used extended carboxylate-TTFs to produce porous frameworks, ${ }^{96,97}$ which showed a close dependence between the conductivity and the strength of the S..S interactions between neighbouring TTF cores which can be tuned by changing the radii of the metallic centers. This series of semiconductive MOFs with tetrathiafulvalene tetrabenzoate (TTFTB) ligands show moderate porosities with BET values around $600 \mathrm{~m}^{2} \mathrm{~g}^{-1}$, and rather moderate conductivities (Fig. 3). Dincă also accessed a different MOF topology using lanthanides as metal ions, but did not increase conductivity values. ${ }^{98}$

The synthesis of new conductive TTF-based MOFs using crystal engineering is key to deliver the expected properties. In order to get good charge carrier mobilities and conductivity values, the relative orientation and the distance between the aromatic groups of the linkers has to be within certain limits for good alignment and sufficient orbital overlapping between the adjacent ligands. On paper, this could be achieved through the restrictions imposed by choosing the appropriate secondary building units (SBUs) through coordination chemistry. However, recent works by $\mathrm{Zhou}^{99}$ and Dincă ${ }^{100}$ point to the fact that inter-linker $\pi-\pi$ interactions might be dominant over the formation of common inorganic SBUs in determining the final packing of the solid. As a result, metallic and superconductive
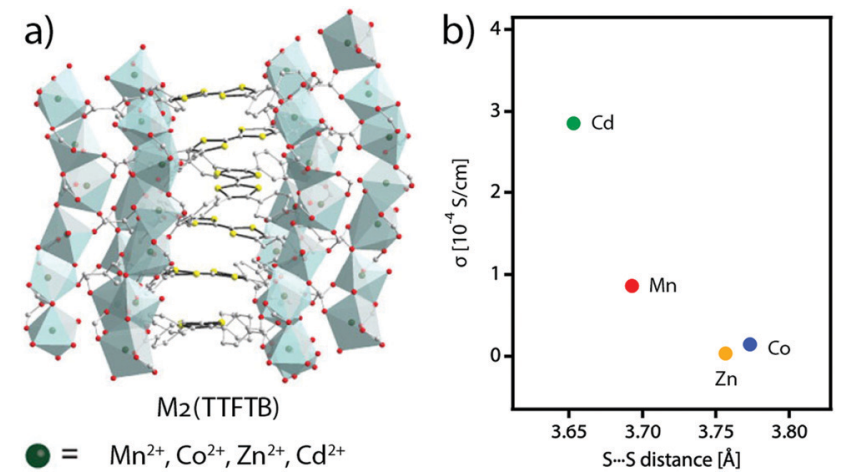

Fig. 3 Example of through-space conductivity with TTF derivatives as ligands highlighting the importance of short contacts between the TTF motifs. (a) Structure of the $M_{2}(\text { TTFTB })_{2}$ MOF reported by Dincă and co-workers. (b) Correlation between the TTF motifs S...S distances and electrical conductivity in $M_{2}$ (TTFTB). Adapted from ref. 97 with permission from the American Chemical Society, copyright 2015. 
behaviours, reminiscent of TTF organic salts, have not yet been reported in TTF-based MOFs, in spite of their potential to surpass those of $2 \mathrm{D} \pi$-conjugated MOFs (see below).

Other than TTF, another case in which close $\pi-\pi$ interactions between ligands can induce high charge carrier mobilities is the In(III)-isophthalate-based MOF prepared by Panda et al. ${ }^{101}$ The presence of the $\pi-\pi$ stacking arrangement among the phenyl rings of the ligands facilitates the high charge carrier mobility $\left(\mu=4.6 \times 10^{-3} \mathrm{~cm}^{2} \mathrm{~V}^{-1} \mathrm{~s}^{-1}\right.$ at $\left.V_{\mathrm{G}}=-40 \mathrm{~V}\right)$ displayed by a fieldeffect transistor (FET) fabricated with this 2D MOF, the first realization of a MOF-FET ever reported. Another example of through-space conductivity is NNU-27, a 3D MOF based on $\mathrm{Zn}^{\mathrm{II}}$ and 4,4'-(anthracene-9,10-diylbis(ethyne-2,1-diyl))dibenzoic acid ligands. The $\pi-\pi$ stacking of the anthracene cores provides a conjugated pathway for charge carriers $\left(\sigma=1.3 \times 10^{-3} \mathrm{~S} \mathrm{~cm}^{-1}\right){ }^{102}$ The addition of the same anthracene core to MOF-74 also provided through-space conductivity to a series of MOFs with different metals. ${ }^{103}$ Similar charge transport paths occur for $\left[\mathrm{Zn}_{2}(\mathrm{BPPTzTz})_{2}(\mathrm{tdc})_{2}\right]^{n}$, (BPPTzTz = 2,5-bis(4-(pyridine-4-yl)phenyl)thiazolo $[5,4-d]$ thiazole), a mixed-valence frameworks in which electrons travel through the cofacial thiazolo[5,4- $d]$ thiazole units. ${ }^{104}$ Yamashita and co-workers also presented the 2D MOF $\left[\mathrm{Cd}(\mathrm{NDI}-\mathrm{py})\left(\mathrm{OH}_{2}\right)_{4}\right]\left(\mathrm{NO}_{3}\right)_{1.3 \pm 0.1} \cdot n \mathrm{DMA}$, that yields a conductivity in the order of $10^{-3} \mathrm{~S} \mathrm{~cm}^{-1}$ thanks to through-space conduction via the face-to-face helical $\pi$-stacked columns of NDI cores. ${ }^{105}$ Dincă reported a different case of charge transport throughspace with the MOF ZnNDI (NDI = naphthalene diimide). The redox active ligand NDI can undergo a reversible reduction into a radical form, which creates a conductive pathway for charge carriers along the stacked naphthalene moieties. ${ }^{106}$ The measured conductivity of the fully reduced framework was not very high $(\sigma=2 \times$ $10^{-7} \mathrm{~S} \mathrm{~cm}^{-1}$ ), but this is the first example of a 3D MOF in which conductivity can be reversibly modulated with a redox reaction.

2.2.2. Through-bond conductivity. For the through-bond conductivity formalism the charge transport happens via metal nodes and linkers. Thus, it requires a certain degree of covalency in the bonding interactions between conjugated organic ligands and metal ions that contain loosely bound electrons. Also, both orbital symmetry and energy overlap between them are needed to promote good charge transport and higher conductivity values. When this overlap is poor, we can have systems based on localized states that still exhibit electrical conductivity due hopping mechanism. ${ }^{107}$ Vital to such a mechanism is the need for the redox centers to have similar or the same reduction potentials and individual immobilized units to be close enough for efficient hopping. Otherwise, an entire molecular component can act as an insulating block to electronic communication across the material. This is the case for the $2 \mathrm{D}$ honeycomb-like $\mathrm{CP}\left[\left\{\mathrm{Rh}_{2}(\text { acam })_{4}\right\}_{3}\left(\mu_{3}-\mathrm{Cl}\right)_{2}\right] \cdot 4 \mathrm{H}_{2} \mathrm{O}$ (acam $=$ acetamide) based on mixed valent $\left[\mathrm{Rh}_{2}(\text { acam })_{4}\right]$ units, which displayed a conductivity of $2 \times 10^{-7} \mathrm{~S} \mathrm{~cm}^{-1} \cdot{ }^{108}$ A 3D diamond-like network built with the same $\left[\mathrm{Rh}_{2}(\mathrm{acam})_{4}\right]$ dimers but connected via a $\mu_{4}$-I bridges instead of $\mu_{3}-\mathrm{Cl}$, displayed much higher conductivity $\left(\sigma=1.4 \times 10^{-3} \mathrm{~S} \mathrm{~cm}^{-1}\right)$. Electron paramagnetic resonance (EPR) results indicated that electrons hop over the $\mathrm{Rh}_{2}$ units with a low activation barrier. Dehydration breaks the symmetry, thus hindering electron delocalization for a drop in conductivity of six orders of magnitude. Another example of conductive CPs based on mixed valency is $\left[\mathrm{Cu}_{2} \mathrm{Br}(\mathrm{IN})_{2}\right]_{n}$ (IN = isonicotinato), a $2 \mathrm{D} \mathrm{CP}$ formed by pairs of $\mathrm{Cu}^{\mathrm{I} / \mathrm{II}}$ ions bridged by two IN ligands and a bromide anion and a conductivity of $1.2 \times 10^{-5} \mathrm{~S} \mathrm{~cm}^{-1} .109$

Entering the MOF category, we find $\mathrm{Fe}^{\mathrm{II}}-\mathrm{Fe}^{\mathrm{III}}$ and $\mathrm{Ru}^{\mathrm{II}}-\mathrm{Ru}^{\mathrm{III}}$ Prussian blue analogues (PBAs) prepared by Long and collaborators in 2009. ${ }^{110}$ PBAs are monoclinic, cubic, or rhombohedral frameworks of cyano-bridged transition metal ions with or without alkali counterions. ${ }^{111}$ In the case of highly symmetrical cubic networks $\mathrm{Fe}_{4}\left[\mathrm{Ru}(\mathrm{CN})_{6}\right]_{3} \cdot 18 \mathrm{H}_{2} \mathrm{O}$ and $\mathrm{K}_{1.2} \mathrm{Ru}_{3.6}\left[\mathrm{Ru}(\mathrm{CN})_{6}\right]_{3} \cdot 16 \mathrm{H}_{2} \mathrm{O},{ }^{110}$ hopping between $\mathrm{Ru}$ or $\mathrm{Fe}$ sites is quite favourable for an overall electric conductivity ranging from $5.7 \times 10^{-3} \mathrm{~S} \mathrm{~cm}^{-1}(\mathrm{Ru})$ to $5.6 \times$ $10^{-5} \mathrm{~S} \mathrm{~cm}^{-1}$ (Fe). Furthermore, they showed to be microporous with BET surface areas of $325(\mathrm{Ru})$ and $550(\mathrm{Fe}) \mathrm{m}^{2} \mathrm{~g}^{-1}$. The mixed valence conductivity also opened the door up for modulable conductivity in systems with metal ions susceptible to easy oxidation such as iron triazolate, tetrazolate and pyrazolate MOFs. Four-probe measurements of a pressed pellet of polycrystalline $\mathrm{Fe}^{\mathrm{II}}\left(\mathrm{C}_{2} \mathrm{~N}_{3} \mathrm{H}_{2}\right)_{2}$ yielded a conductivity value of $0.77 \times$ $10^{-4} \mathrm{~S} \mathrm{~cm}^{-1}$. $^{112}$ After exposure to $\mathrm{I}_{2}$, the conductivity value slightly increased to $1 \times 10^{-3} \mathrm{~S} \mathrm{~cm}^{-1}$ due to $\mathrm{Fe}^{\mathrm{II}}$ being oxidized to $\mathrm{Fe}^{\mathrm{III}}$ for overall mixed valence conductivity. The effect was much pronounced in $\left[\mathrm{Fe}(\mathrm{tri})_{2}\left(\mathrm{BF}_{4}\right)_{x}\right]$ (tri $=1,2,3$-triazolate) that increased eight orders of magnitude in conductivity after exposure to $\mathrm{O}_{2}$ (Fig. 4). ${ }^{113}$ Dincă and collaborators observed a similar change for $\mathrm{Fe}_{2}(\mathrm{BDT})_{3} \quad\left[\mathrm{H}_{2} \mathrm{BDT}=5,5^{\prime}\right.$-(1,4-phenylene $)$ bis $\left(1 H^{-}\right.$ tetrazole)] with air exposure over five orders of magnitude and exceeding $1 \mathrm{~S} \mathrm{~cm}^{-1}$, the highest conductivity so far for a 3D MOF. ${ }^{114}$ Moreover, depending on the exposure time, the $\mathrm{Fe}^{\mathrm{II}}$ to $\mathrm{Fe}^{\mathrm{III}}$ oxidation could be modulated along with conductivity to reach a maximum after 30 days. Long and collaborators achieved a similar redox modulated conductivity by controlling the chemical reduction of $\mathrm{Fe}_{2}(\mathrm{BDP})_{3}$ (BDP = 1,4-benzenedipyrazolate) and reached a maximum conductivity of $2.5 \times 10^{-2} \mathrm{~S} \mathrm{~cm}^{-1} \cdot{ }^{115} \mathrm{Hupp}$ and co-workers also turned the insulating undoped NU-1000 MOF conductive via electrochemical oxidation of part of the tetraphenylpyrene linkers. Then, the redox hoping charge transport was analysed for two different crystal orientations, parallel
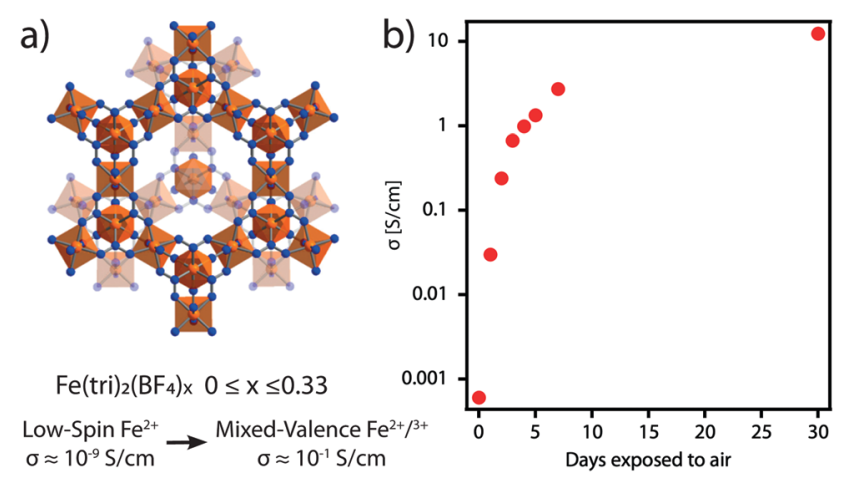

Fig. 4 Modulable conductivity in a Fe(tri $)_{2}\left(\mathrm{BF}_{4}\right)_{x} \mathrm{MOF}(\mathrm{a})$ that increases conductivity with $\mathrm{Fe}^{\prime \prime}$ oxidation to $\mathrm{Fe}^{\mathrm{III}}$ in atmospheric conditions (b). Adapted from ref. 113 with permission from the American Chemical Society, copyright 2018. 
and perpendicular to the pore channels, revealing a highly anisotropic conduction in this MOF. ${ }^{116}$

The hopping charge transport mechanism between redox metal centers is also present in metal tetracarboxylate porphyrin MOFs and CPs. ${ }^{117}$ Morris and collaborators prepared micrometric thin films of CoPIZA onto fluorine-doped tin oxide (FTO) via solvothermal synthesis containing Co ${ }^{\mathrm{II}} \mathrm{TCPP}$ (TCPP $=5,10,15,20$-tetrakis(4-carboxyphenyl)porphyrinato) struts bound by linear trinuclear $\mathrm{Co}^{\mathrm{II}}$ carboxylate clusters to form a porous network with large channels. ${ }^{118}$ The bonds in the Co clusters are carboxylate in nature, which are known to be insulating. Therefore, it is unlikely that sufficient HOMO-LUMO overlap exists between the two molecular components to justify a band structure. Hence, CoPIZA was proposed to be a 3D network of individual redox centers. The measured conductivity, determined by the reciprocal of the area and thickness corrected low frequency intercept of the Nyquist plot, was $3.62 \times 10^{-8} \mathrm{~S} \mathrm{~cm}^{-1}$, this places the system in the range of wide band gap semiconductors. Given the almost insulating behaviour of CoPIZA/FTO, charge transfer is likely to occur via a redox hopping mechanism as proven by electrochemical and spectroelectrochemical analysis.

Regarding the role played by the ligands, as in the case of the metal nodes, CPs with mixed valent aromatic ligands usually record high conductivities like the anilate-based MOFs $\left(\mathrm{NBu}_{4}\right)_{2} \mathrm{Fe}_{2}(\mathrm{dhbq})_{3}(\mathrm{dhbq}=2,5$-dioxidobenzoquinone; $\sigma=1 \times$ $\left.10^{-2} \mathrm{~S} \mathrm{~cm}^{-1}\right)^{119}$ and $\left(\mathrm{Me}_{2} \mathrm{NH}_{2}\right)_{2}\left[\mathrm{Fe}_{2}\left(\mathrm{Cl}_{2} \mathrm{An}\right)_{3}\right] \cdot 2 \mathrm{H}_{2} \mathrm{O} \cdot 6 \mathrm{DMF}(\mathrm{An}=$ anilate; $\left.\sigma=1.4 \times 10^{-2} \mathrm{~S} \mathrm{~cm}^{-1}\right),{ }^{120}$ which are much more conductive that their non-redox active oxalate analogues. ${ }^{121}$ Furthermore, even when the intervalence charge transfer (IVCT) occurs between metal centers instead, like in $\left[\left(\mathrm{H}_{3} \mathrm{O}\right)\left(\mathrm{H}_{2} \mathrm{O}\right)(\text { phenazine })_{3}\right]\left[\mathrm{Fe}^{\mathrm{II}} \mathrm{Fe}^{\mathrm{III}}\right.$ $\left.\left(\mathrm{Cl}_{2} \mathrm{An}\right)_{3}\right]^{122}$ and [tris(amino)-guanidinium $]\left[\mathrm{Fe}^{\mathrm{II}} \mathrm{Fe}^{\mathrm{III}}(\mathrm{ClCNAn})_{3}\right]{ }^{123}$ anilate ligands yield MOFs with higher conductivities due to the much lower electron hopping barrier in the anilate complexes with respect to oxalates. ${ }^{123}$ These materials also highlight the interplay between the mixed valency required for high conductivity and the presence of unpaired electrons that favours high temperature magnetic ordering. This conflicting relationship has been shown in these semiquinoid frameworks with post-synthetic redox reactions. Systems with mixed valent ligands such as $\left(\mathrm{NBu}_{4}\right)_{2}-$ $\mathrm{Fe}_{2}(\mathrm{dhbq})_{3}$ and $\left(\mathrm{Me}_{2} \mathrm{NH}_{2}\right)_{2}\left[\mathrm{Fe}_{2}(\mathrm{ClAn})_{3}\right]$ have relatively high conductivities, but low magnetic ordering temperatures. ${ }^{119,120}$ However, post-synthetic chemical reduction of a part or all the ligands in these frameworks to their fully reduced radical forms $\left(\mathrm{dhbq}^{3-}\right.$ • and $\mathrm{Cl}_{2} \mathrm{An}^{3-\bullet}$ ), yields compounds with higher magnetic ordering temperatures and lower conductivities. Curiously, the 2D $\mathrm{Mn}^{\mathrm{II}}$ benzoquinoid framework $\left(\mathrm{Me}_{4} \mathrm{~N}\right)_{2}\left[\mathrm{Mn}_{2}\left(\mathrm{Cl}_{2} \mathrm{An}\right)_{3}\right] \cdot 3.2 \mathrm{Et}_{2} \mathrm{O}$ shows the opposite behaviour with regards to electrical conductivity. Chemical reduction to its radical form $\mathrm{Na}_{3}\left(\mathrm{Me}_{4} \mathrm{~N}\right)_{2}\left[\mathrm{Mn}_{2}\left(\mathrm{Cl}_{2} \mathrm{An}^{3-\bullet}\right)_{3}\right] \cdot 3.9 \mathrm{THF}$, provokes a magnetic transition from paramagnet to permanent magnet with a characteristic temperature of $T_{\mathrm{c}}=41 \mathrm{~K}$, but at the same time the room temperature conductivity increases by more than five orders of magnitude. ${ }^{124}$ As mentioned in Section 2.1.2, redox modulation of the electrical conductivity has also been reported for TTF-based MOFs. Zuo and collaborators prepared $\left\{\left[\mathrm{Fe}(\mathrm{dca})_{2}\right]\left[\mathrm{TTF}(\text { py })_{4}\right] 0.5 \cdot 0.5 \mathrm{CH}_{2} \mathrm{Cl}_{2}\right\}_{n}$ and $\left\{[\mathrm{Fe}(\mathrm{dca})]\left[\mathrm{TTF}-(\mathrm{py})_{4}\right] \cdot \mathrm{ClO}_{4}\right.$. $\left.\mathrm{CH}_{2} \mathrm{Cl}_{2} \cdot 2 \mathrm{CH}_{3} \mathrm{OH}\right\}_{n}$ (dca = dicyanamide); TTF-(py) $)_{4}=((4$-pyridyl)tetrathiafulvalene). The first is an interpenetrated 3D network, whilst the second is a MOF formed by $\left\{\mathrm{Fe}\left[\mathrm{TTF}(\mathrm{py})_{4}\right]\right\}_{n}$ undulating layers joined by dca ligands (Fig. 11a). ${ }^{73}$ Oxidation of the TTF-(py) ${ }_{4}$ linkers using $\mathrm{I}_{2}$ increased the conductivity in both compounds by 2-3 orders of magnitude, probably thanks to IVCT between the oxidized $\mathrm{TTF}^{\bullet+}$ radical units. Interestingly, the second compound maintained SCO bistability after oxidation (Fig. 11b, see Section 3.2.). ${ }^{73}$ Furthermore, IVCT is not unique to the through-bond mechanism, as it has also been observed to occur through-space between cofacial thiazolo[5,4- $d$ ] thiazole units in $\mathrm{Zn}^{\mathrm{II}}$ MOFs. ${ }^{104}$ Additionally, the use of aromatic ligands and transition metal ions can result in a strong hybridization of the $\pi$-d orbitals which facilitates charge transport and favours high conductivity. For example, this was the case for the layered $\mathrm{CP} \mathrm{CrCl}_{2}(\mathrm{pz})_{2}$ (pz: pyrazine), which showed a conductivity of $3.2 \times 10^{-2} \mathrm{~S} \mathrm{~cm}^{-1}$ through thermally activated hopping mechanism involving both $\mathrm{pz} \pi$ and $\mathrm{Cr}^{\mathrm{III}} 3 \mathrm{~d}$ orbitals. ${ }^{125}$

The interplay between conductivity (i.e. electronic structure) and structure (i.e. geometry) makes it difficult to extrapolate conductivity relationships among CP series. The most important factors governing conductivity are the number of charge carriers and its mobility. In a naive manner, more polarizable atoms (lower electronegativity, better orbital overlap) should render higher conductivities. The polarizability advantage has been observed for CPs and MOFs alike, with ligands that coordinate metal centers through S-donor atoms usually reaching higher conductivity values than their $\mathrm{O}$ and $\mathrm{N}$ equivalents. Good examples of this are 2D CPs like $\mathrm{Cu}-\mathrm{HT}$ (HT: 4-hydroxythiophenol), with one of the highest conductivity values for a $\mathrm{CP}\left(\sigma=120 \mathrm{~S} \mathrm{~cm}^{-1}\right),{ }^{126}$ and the metalorganic graphene analogue (MOG) family (see below). The relative importance of linkers with polarizable bridging atoms and loosely bound charge carriers was analyzed by Dincă and collaborators with a series of MOF-74 type materials. ${ }^{127}$ Replacement of $\mathrm{Mn}^{\mathrm{II}}$ by $\mathrm{Fe}^{\mathrm{II}}$ in $\mathrm{Fe}_{2}$ (DSBDC) (MOF-74, DOBDC ${ }^{4-}=2,5$-dihydroxybenzene1,4-dicarboxylate) yielded a million-fold conductivity enhancement. Whilst varying the bridging atom of the DEBDC ligand $(\mathrm{E}=\mathrm{O}$ or $\mathrm{S})$ only changed the bulk electrical conductivity by around one order of magnitude, $3.9 \times 10^{-6} \mathrm{~S} \mathrm{~cm}^{-1}$ for $\mathrm{Fe}_{2}$ (DSBDC) versus and $3.2 \times$ $10^{-7} \mathrm{~S} \mathrm{~cm}^{-1}$ for $\mathrm{Fe}_{2}(\mathrm{DOBDC})$. Moreover, these values are still $\sim 6$ orders of magnitude higher than those of the $\mathrm{Mn}^{2+}$ analogues, $\mathrm{Mn}_{2}$ (DEBDC). Because the metals are of the same formal oxidation state, the increase in conductivity was attributed to the loosely bound $\mathrm{Fe}^{2+} \beta$-spin electron. The addition of a single electron per metal ion (i.e., substitution of $\mathrm{d}^{5} \mathrm{Mn}^{2+}$ for $\mathrm{d}^{6} \mathrm{Fe}^{2+}$ ) had a much more pronounced positive effect on conductivity than changing the bridging atom from $\mathrm{O}$ to $\mathrm{S}$, indicating that the polarizability and electronic configuration of the metal ions plays the most important role in charge conduction in this class of materials.

2.2.3. Metal-organic graphene analogues: highly conjugated 2D MOFs and CPs. Amongst the conductive MOFs available, the most prolific class in terms of the number of reports, conductivity and charge carrier mobility values to date are MOGs, also referred to with the more generic term $2 \mathrm{D} \pi$-conjugated MOFs. ${ }^{128}$ Thus far, they are the most promising candidates for integration into electronic devices. These materials are formed by neutral $2 \mathrm{D}$ planar layers composed by divalent metal ions linked to aromatic or polyaromatic ligands with $\mathrm{Se}, \mathrm{S}, \mathrm{N}$, or $\mathrm{O}$ as bridging atoms. The 
layered structure results strong in-plane charge delocalization and extended $\pi$-conjugation along the metal-organic sheets. The term MOG was originally coined by Dincă and collaborators in the communication that reported the synthesis of $\mathrm{Ni}_{3}(\mathrm{HITP})_{2}$ (HITP: 2,3,6,7,10,11-hexaiminotriphenylene), due to its low bandgap and good electrical conductivity enabled by full charge delocalization in the $2 \mathrm{D}$ plane. ${ }^{129}$ Depending on the choice of aromatic backbone (benzene, coronene, triphenylene or truxene) and ortho-substituted hetero atoms $(\mathrm{O}, \mathrm{N}, \mathrm{S}$ or Se) of the ligand, and divalent metal ion $\left(\mathrm{Fe}^{\mathrm{II}}, \mathrm{Co}^{\mathrm{II}}, \mathrm{Ni}^{\mathrm{II}}, \mathrm{Cu}^{\mathrm{II}}, \mathrm{Pd}^{\mathrm{II}}\right.$ or $\left.\mathrm{Pt}^{\mathrm{II}}\right)$, the final MOG can have hexagonal honeycomb or Kagome in-plane lattices. Likewise, the stacking of the 2D layers can be eclipsed (AA), slipped-parallel (AB) or staggered $\left(\mathrm{AA}^{-1}\right)$. In general, the crystallinity of MOGs is somewhat poor due to the fast assembly kinetics caused by strong interlinker $\pi-\pi$ interactions. Therefore, it has been extremely difficult to obtain single crystals that are large enough for single crystal X-ray diffraction (SCXRD), except for one case. ${ }^{130}$ Thus, most of the reported structures are based on powder X-ray diffraction (PXRD) analysis of microcrystalline powders coupled with structural simulations, density functional theory (DFT) calculations, high resolution transmission electron microscopy (HRTEM) or/and X-ray absorption fine structure (EXAFS) analysis. From a structural point of view, this has made it difficult to obtain a definitive structural arrangement in some reports, especially to distinguish between out-of-plane dispositions which can be very close energetically. Fig. 5 shows the variety of structural arrangements of this family of materials.

Regarding benzene linkers (Fig. 5a and b), the honeycomb assembly with hexagonal pores is the most common in-plane framework disposition for ligands benzenehexathiol (BHT), hexaiminobenzene (HIB), hexahydroxybenzene ( $\mathrm{HHB}$ ) and finally 1,3,5-triaminobenzene-2,4,6-trithiol, referred to as bis(iminothiolato) a)

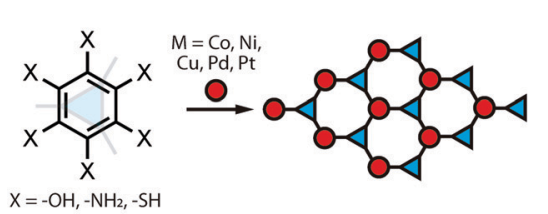

b)

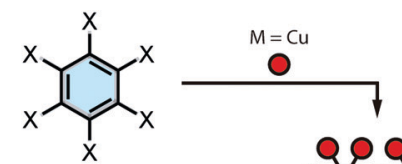

$X=-\mathrm{SH},-\mathrm{SeH}$

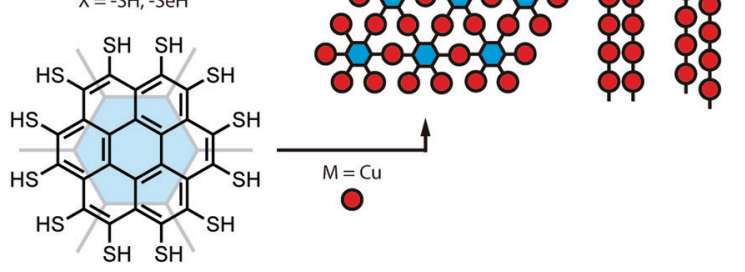

d)<smiles>Oc1cc2cc3c4cc(O)c(O)cc4c4cc(O)c(O)cc4c3c3cc(O)c(O)cc3c-2c1</smiles>

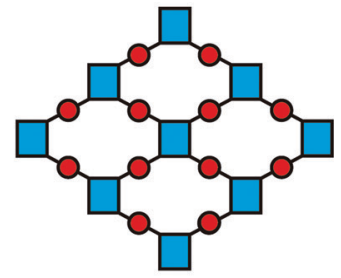

e)

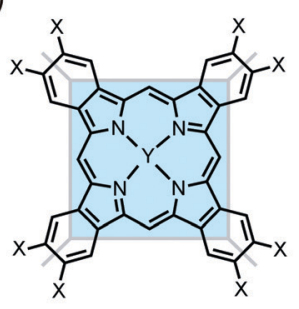

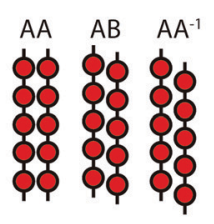

c)

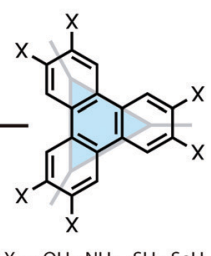

$X=-\mathrm{OH},-\mathrm{NH}_{2},-\mathrm{SH},-\mathrm{SeH}$
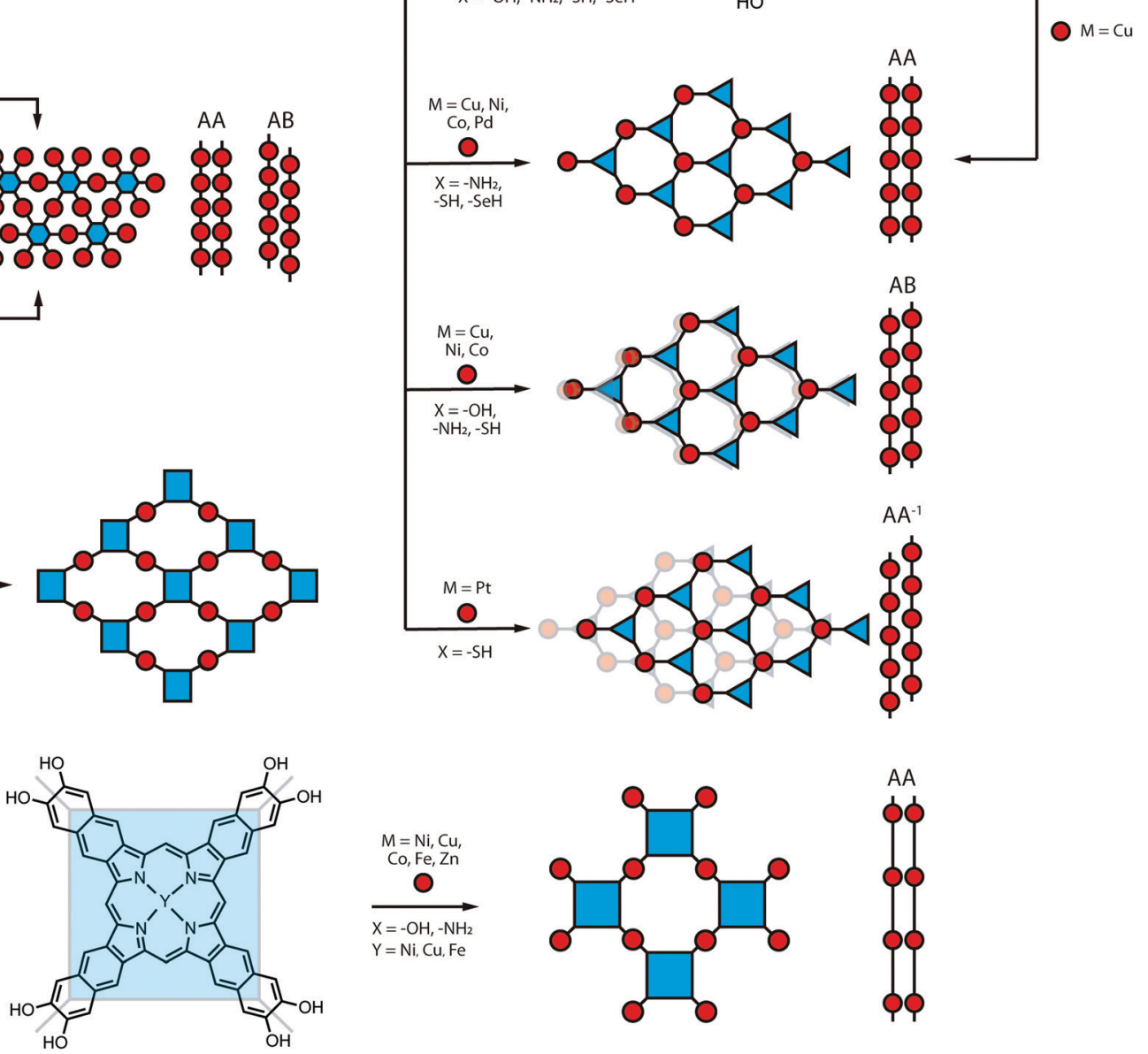
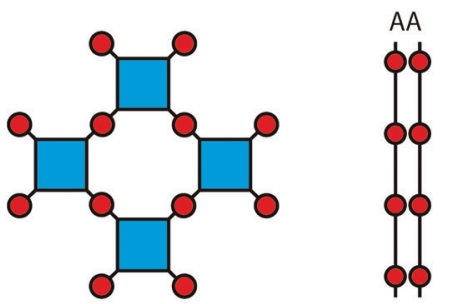

Fig. 5 Different in-plane lattices and out-of-plane packing of 2D $\pi$-conjugated MOFs based on cores of (a) benzene, (b) benzene and coronene, (c) triphenylene and truxene, (d) $D_{2}$ symmetric catechol and (e) phthalocyanine and naphthalocyanine ligands with hydroxy, amine, thiol or selenol binding groups. 
(IT) or bis-(aminothiolato) (AT) depending on the oxidation state of the bridging $\mathrm{N}$ group. However, there are a few notable exceptions, one is the $\left[\mathrm{Cu}_{3}\left(\mathrm{C}_{6} \mathrm{~S}_{6}\right)\right]_{n}$ or $\mathrm{Cu}-\mathrm{BHT}$ Kagome network originally reported by $\mathrm{Xu}$, Zhu and collaborators. ${ }^{131}$ Its fully filled honeycomb structure does not generate pore openings so $\mathrm{Cu}-\mathrm{BHT}$ is non-porous and therefore a CP. We encounter the same situation with its selenium derivate $\mathrm{Cu}-\mathrm{BHS}$ (BHS = benzenehexaselenolate), which presents the same in-plane arrangement. ${ }^{132}$ Another exception is the recently reported $\left[\mathrm{Ag}_{5}\left(\mathrm{C}_{6} \mathrm{~S}_{6}\right)\right]_{n}$ or $\mathrm{Ag}$-BHT, a lamellar $\mathrm{CP}$ with fully filled topology. ${ }^{133}$ The reported structure has alternatively stacked 2D $\mathrm{Ag}-\mathrm{S}$ networks and layers composed of 1D metal-dithiolene polymer with octahedral and square planar $\mathrm{Ag}^{\mathrm{I}}$ ions. Vaid and co-workers also published a CP semiconductor with $\mathrm{Pb}^{\mathrm{II}}$ and BHT. In this case, there is a 3D connectivity in between consecutive layers of $\mathrm{Pb}^{\mathrm{II}}$ ions and BHT ligands in a similar structure to that of Pbs. ${ }^{134}$ The rest of compounds known to date with benzene linkers have a hexagonal honeycomb lattices and include Ni-BHT, ${ }^{135} \mathrm{Co}-\mathrm{BHT},{ }^{136} \mathrm{Pd}-\mathrm{BHT},{ }^{137}$ Pt-BHT, ${ }^{138}$ Ni-IT, ${ }^{139}$ Ni-AT, ${ }^{140}$ Ni-HIB, ${ }^{141,142}$ Cu-HIB, ${ }^{141,142}$ Co$\mathrm{HIB}^{141,143}$ and $\mathrm{Co}-\mathrm{HHB},{ }^{144}$ all with a general formula of $\left[\mathrm{M}_{3}\left(\mathrm{C}_{6} \mathrm{X}_{6}\right)_{2}\right]_{n}$ where $\mathrm{M}=\mathrm{Ni}, \mathrm{Co}, \mathrm{Cu}, \mathrm{Pd}$ or $\mathrm{Pt}$ and $\mathrm{X}=\mathrm{Se}, \mathrm{S}, \mathrm{NH}, \mathrm{NH}_{2}$ or $\mathrm{O}$.

As mentioned above, it is difficult to distinguish between interlayer stackings in MOGs. For the Cu-BHT Kagome network, the stacking configuration was initially reported as an undistinguishable mixture of $\mathrm{AA}$ and $\mathrm{AB}$ patterns, ${ }^{131}$ but another PRXD analysis of a sample with improved crystallinity showed that the eclipsed packing is a better fit to the data. ${ }^{145}$ Interestingly, of the benzene hexagonal honeycomb MOGs, only Co-HIB ${ }^{143}$ shows an eclipsed stacking. $\mathrm{Ni}-\mathrm{BHT}^{135}$ and NiAT ${ }^{140}$ stack in a staggered $\left(\mathrm{AA}^{-1}\right)$ disposition, whilst Pt-BHT, ${ }^{138} \mathrm{Ni}-\mathrm{HIB},{ }^{142} \mathrm{Cu}-\mathrm{HIB}^{142}$ and $\mathrm{Co}-\mathrm{HHB}^{144}$ have slipped-parallel interlayer arrangement and the disposition for Co-BHT has not been reported. The other benzene-based MOG with a Kagome lattice, $\mathrm{Cu}$-BHS, was better fitted to a slipping $\mathrm{AA}^{\prime}$ configuration. ${ }^{132}$

The structural variety of benzene MOGs is also present in their triphenylene equivalents (Fig. $5 \mathrm{c}$ ). These ligands with four aromatic rings are 2,3,6,7,10,11-hexahydroxytriphenylene (HHTP), HITP, 2,3,6,7,10,11-hexathiotriphenylene (HTTP) and 2,3,6,7,10,11triphenylenehexaselenol (TPHS). All of them yield in-plane hexagonal honeycomb lattices with $\mathrm{Fe}^{\mathrm{II}}, \mathrm{Co}^{\mathrm{II}}, \mathrm{Ni}^{\mathrm{II}}, \mathrm{Cu}^{\mathrm{II}}$ and $\mathrm{Pt}^{\mathrm{II}}$ as metal centres. Concerning their out-of-plane stacking, the first 2D frameworks published as MOGs were Dincă's $\mathrm{Ni}_{3}(\mathrm{HITP})_{2}{ }^{129}$ and $\mathrm{Cu}_{3}(\mathrm{HITP})_{2}{ }^{146}$ which were both originally reported as having slipped-parallel disposition, as well as the THTA-Co MOG built with mixed HITP and HTTP ligands. ${ }^{147}$ On the other hand, $\mathrm{Co}_{3}(\mathrm{TPHS})_{2},{ }^{148} \mathrm{Co}_{3}(\mathrm{HTTP})_{2},{ }^{149} \mathrm{Ni}_{3}(\mathrm{HTTP})_{2}{ }^{150}$ and $\mathrm{Cu}_{3}(\mathrm{HTTP})_{2}{ }^{151}$ layers are eclipsed and in the case of $\mathrm{Pt}_{3}(\mathrm{HTTP})_{2}$ they are staggered. ${ }^{152}$ Additionally, $\mathrm{Ni}_{3}(\mathrm{HITP})_{2}$ was latter clarified as having an eclipsed or near-eclipsed staking as well, thanks to a single crystal study ${ }^{153}$ Sticking with materials formed by the HTTP ligand, we can find a rare example of a 3D MOFs prepared with $\mathrm{Pb}^{\mathrm{II}}$ metal ions: $\mathrm{Pb}$ HTTP. ${ }^{154}$ This 3D gyroidal net features a formula $\left[\mathrm{Pb}_{3} \mathrm{OH}_{0.5^{-}}\right.$ (HTTP) $]^{1.5-}$ in which trigonal $\mathrm{Pb}_{3}\left(\mu_{3}-\mathrm{O}\right)$ cores are bonded to tritopic HTTP linkers, generating a three-connected network of the gyroid topology (srs net) as determined by SCXRD. Even though this material is semiconducting $\left(\sigma=1.1 \times 10^{-6} \mathrm{~S} \mathrm{~cm}^{-1}\right)$, it cannot be considered a MOG owing to its 3D topology and large bandgap (1.7 eV).
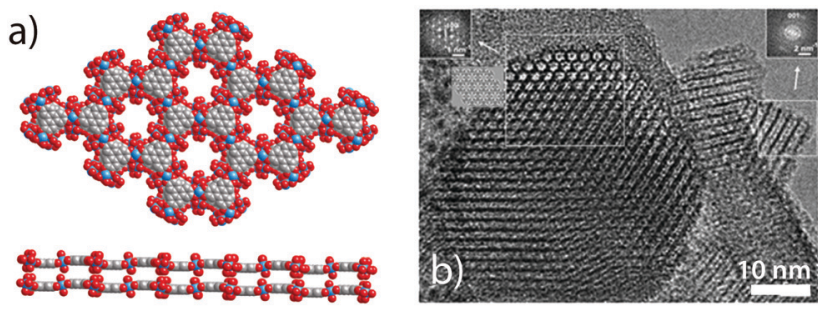

Fig. 6 (a) Crystal structure of Co-CAT-1 along the $c$ axis. (b) HR-TEM image of nanocrystals of the isostructural Ni-CAT-1. Reproduced from ref. 130 with permission from the American Chemical Society, copyright 2012.

Thus far, all the above mentioned MOGs are composed by divalent metal ions 4-coordinated in a square planar geometry. Yet, there are a few cases where metal centers have octahedral geometries with triphenylene linkers occupying the equatorial positions and axial monodentate ligands occupying the axial ones. Focusing on MOGs prepared with the HHTP ligand, the first 2D $\pi$-conjugated MOFs ever reported are the M-CAT-1 series. ${ }^{130}$ A 2012 paper by Yaghi and collaborators featured three MOFs: Co-CAT-1, NiCAT-1 and Cu-CAT-1. Co-CAT- 1 and Ni-CAT-1 have the same structure which consists of octahedral $\mathrm{Co}^{\mathrm{II}}$ and $\mathrm{Ni}^{\mathrm{II}}$ ions coordinated to HHTP ligands and $\mathrm{H}_{2} \mathrm{O}$ molecules to form two different kinds of layers, one formed by an extended $\mathrm{Co}_{3}(\mathrm{HHTP})_{2}\left(\mathrm{H}_{2} \mathrm{O}\right)_{6}$ network with hexagonal pores and the other by discrete $\mathrm{Co}_{3}(\mathrm{HHTP})\left(\mathrm{H}_{2} \mathrm{O}\right)_{12}$ units (Fig. 6). The two types of layers are alternatively stacked in the out-ofplane directions in an eclipsed fashion with the HHTP molecules in each layer rotated $60^{\circ}$ with respect to each other. Also, the two axial water ligands of these discrete complexes are hydrogen bonded to oxygen atoms of the HHTP in the neighbouring layers, which causes the hexagonal layers to be slightly corrugated. All this structural information was obtained thanks to synchrotron SCXRD that allowed to solve the crystal structure of Co-CAT-1. Unfortunately, the crystal structure of Cu-CAT-1 could not be solved, but PXRD data pointed to a different structure to that of Co-CAT- 1 and NiCAT-1. Following reports have shone light on the issue through PXRD, DFT calculations, HRTEM and structural modelling. Like other MOGs with HITP and HTTP ligands, Cu-CAT-1 or $\mathrm{Cu}_{3}(\mathrm{HHTP})_{2}$ has only one type of hexagonal honeycomb layer in which $\mathrm{Cu}^{\text {II }}$ centers are square planar without the presence of axial $\mathrm{H}_{2} \mathrm{O}$ molecules. Initially, there were minor discrepancies in the literature over the interlayer stacking of these layers. According to Xu's ${ }^{155}$ and Martí-Gastaldo's ${ }^{156}$ models they present a slipped-parallel $\mathrm{AB}$ disposition with similar interlayer distances (3.33 ̊ and $3.36 \AA$ respectively), whilst a recent report by Behrens and collaborators describes an eclipsed stacking favoured by attractive metal-metal interactions and with a slightly lower interlayer separation $(3.16 \AA) .{ }^{157}$ Nonetheless, experimental conductivity data together with DFT calculations of the electronic structure pointed towards an $\mathrm{AB}$ stacking as the most likely disposition. ${ }^{158}$ Afterwards, a HRTEM study of exfoliated single crystals by Dincă's group clarified the controversy, hence consecutive Cu-CAT-1 layers are displaced in an undulating fashion which creates a kind of zig-zag pore channel. ${ }^{153}$

Another special type of MOF built with HHTP was just reported by Dincă and collaborators using lanthanide ions. ${ }^{159}$ Hence, LnHHTP $\left(\mathrm{Ln}=\mathrm{La}^{\mathrm{III}}, \mathrm{Nd}^{\mathrm{III}}, \mathrm{Ho}^{\mathrm{III}}, \mathrm{Yb}^{\mathrm{III}}\right)$ have a general formula of 


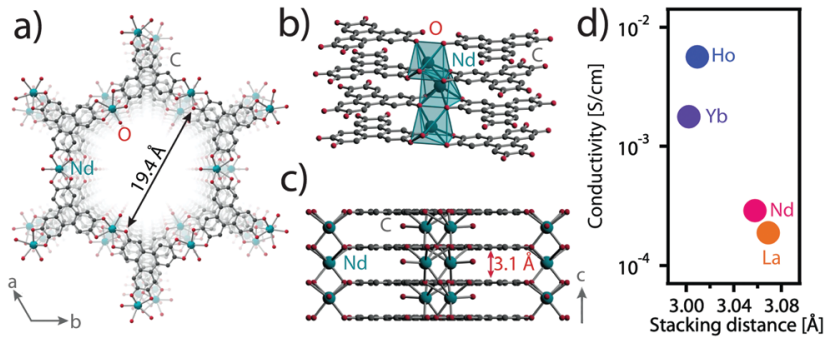

Fig. 7 The 3D connected LnHHTP MOFs. (a) View along the $c$ axis of the crystal structure of NdHHTP. Side views showing the interlayer distance (b) and the heptacoordination of the $\mathrm{Nd}^{\text {III }}$ ions (c). (d) Electrical conductivity of the LnHHTP plotted as a function of their interlayer distances. Adapted from ref. 159 with permission from Springer Nature, copyright 2020.

$\operatorname{Ln}_{1+x} \mathrm{HHTP}\left(\mathrm{H}_{2} \mathrm{O}\right)_{n}(x=0-0.2)$ and crystallize in the same hexagonal honeycomb in-plane arrangement as triphenylene-based MOGs (Fig. 7a). However, in this case there is out-of-plane connectivity through the Ln ions that lie in-between the HHTP layers and are coordinated to six $\mathrm{O}$ atoms of the ligands and one additional water or hydroxide molecules (Fig. 7b and c). Therefore making LnHHTP MOFs 3D systems. Concerning their properties, they present similar porosity to triphenylene-based MOGs with BET values between 200 and $510 \mathrm{~m}^{2} \mathrm{~g}^{-1}$. However, their charge transport characteristics are somewhat unique. DFT calculations indicate that a strongly anisotropic metal-like transport perpendicular to the Ln-HHTP layers and a semiconductive behaviour along them. Although the conductivity measurements were performed using pellets made form polycrystalline materials, the authors related the conductivity values of the different LnHHTP MOFs to their interlayer distances. Conductivity values up to two orders of magnitude higher were obtained for the materials with a shorter interlayer HHTP-to-HHTP distance (Fig. 7d). Thus, in this case the charge carriers would travel perpendicular to the layers in a through-space regime. In contrast with $2 \mathrm{D}$ MOG systems, for which charge transport occurs more favourably in-plane as indicated through comparison of horizontal and vertical devices of Cu-CAT-1 oriented films. ${ }^{160}$

Finally, there are a few special MOG systems with mixed or chemically modified ligands. Kitagawa, Otake and co-workers published a dual-ligand MOG, $\mathrm{Cu}_{3}$ (HHTP)(THQ) (THQ = tetrahydroxy1,4-quinone) that combines triphenylene and benzene-based linkers with $\mathrm{X}=\mathrm{O} .{ }^{161}$ Here, a Cu${ }^{\mathrm{II}}$ ion is coordinated to one HHTP and one THQ. In order to balance the different affinities of both ligands and obtain the dual-ligand MOG, a $\mathrm{Cu}(\mathrm{en})_{2}$ (en = ethylenediamine) complex was used as precursor instead of $\mathrm{a} \mathrm{Cu}^{\mathrm{II}}$ salt like in the rest of Cu-based MOGs. $\mathrm{Cu}_{3}$ (HHTP)(THQ) crystallizes in the same hexagonal in-plane arrangement as its triphenylene-based counterparts and the layers stack in an $\mathrm{AB}$ slipped-parallel disposition according to PXRD and HRTEM analysis. In the other case, Ruoff, Yoo and collaborators prepared a macrocycle derivative of $\mathrm{Ni}_{3}(\mathrm{HITP})_{2}$. NiTAA (TTA = tetraaza[14]annulene) was prepared via an in situ polycondensation reaction of HITP with 1,1,3,3-tetramethoxypropane and a $\mathrm{Ni}^{\mathrm{II}}$ salt to form a 2D hexagonal honeycomb network with imine bonds in the coordination sphere of $\mathrm{Ni}^{\mathrm{II}}$. The out-of-plane disposition of layer fits better with a slipped-parallel stacking mode. ${ }^{162}$
The structural differences commented above, give rise to acute differences in the porosity of MOGs. They are all in the microporous range (pore apertures from 0.2 to $20 \mathrm{~nm}$ ), but the smaller pores of MOGs with benzene linkers logically yield lower BET values. For instance, Ni-HIB, Cu-HIB and Co-HHB have all $\mathrm{AB}$ interlayer stackings and show $\mathrm{BET}$ values up to 4-5 times lower (152, 114 and $143 \mathrm{~m}^{2} \mathrm{~g}^{-1}$ respectively $)^{140,144}$ than the equivalent triphenylene MOGs. Meanwhile, Co-HIB with an eclipsed (AA) stacking gives out a slightly higher value $\left(\mathrm{BET}=240 \mathrm{~m}^{2} \mathrm{~g}^{-1}\right) \cdot{ }^{143}$ Generally, triphenylene MOGs have larger pore apertures and naturally larger BET values. However, they do not clearly correlate well with the interlayer stackings. For instance, $\mathrm{Co}_{3}(\mathrm{HTTP})_{2}$ and $\mathrm{Pt}_{3}(\mathrm{HTTP})$ have similar BET values (370 and $329 \mathrm{~m}^{2} \mathrm{~g}^{-1}$ respectively), ${ }^{149,152}$ but the first one has an eclipsed disposition whilst the second one is staggered. Meanwhile, dual-ligand MOG $\mathrm{Cu}_{3}(\mathrm{HHTP})(\mathrm{THQ})$ has a BET surface area of $441 \mathrm{~m}^{2} \mathrm{~g}^{-1}$ with a slipped-parallel disposition. ${ }^{161}$ It is important to highlight the disparity in the reported BET values, even for the same material, probably due to small experimental differences in synthesis and solvent removal (activation) protocols or the difficulties on controlling the crystallinity of this family of materials. For example, BET surface areas of $284,{ }^{163}$ $348,{ }^{158} 512^{157}$ and $540^{155} \mathrm{~m}^{2} \mathrm{~g}^{-1}$ have been calculated just for $\mathrm{Cu}$-CAT-1 in different reports. In any case, the record BET surface area for a MOG is $630 \mathrm{~m}^{2} \mathrm{~g}^{-1}$ of $\mathrm{Ni}_{3}(\mathrm{HITP})_{2}{ }^{164}$ There are also some minor discrepancies in the pore size thorough the literature (see Table 1).

Regarding the electronic properties of the MOG family, in general conductivity values are comparable to those of typical organic conductors $\left(\sigma>1 \times 10^{-6} \mathrm{~S} \mathrm{~cm}^{-1}\right)$. As previously mentioned, MOGs are a family of very interesting conductive materials due to their high conductivity and low modulable bandgaps. Their extended $\pi$-conjugation along the sheets and the strong $\mathrm{p}-\mathrm{d}$ orbital coupling ${ }^{165}$ results in large in-plane charge delocalization. Furthermore, both metallic nodes and organic linkers can serve as the source of charge carriers thanks to their redox active characters and unpaired electrons. The redox active character of their benzene and triphenylene ligands is well known (Fig. 8), as well as the possibility to form stable radicals. In fact, in most MOGs the ligand gets oxidized to a monoradical with a total charge of -3 in order to achieve charge balance with the divalent metal ion. For Co-CAT-1 this was demonstrated via EPR data, which displays a near-symmetric signal at $g=2.105$ characteristic of a ligand-centered monoradical. Thus, on the basis of the charge balance, the oxidation state of the deprotonated HHTP suggests that each of the three dioxolene fragments is in the semiquinone oxidation state and the Co ions are all divalent. Interestingly, for Cu-based MOGs X-ray photoelectron spectroscopy (XPS) data shows presence of $\mathrm{Cu}^{\mathrm{I}}$ in $\mathrm{Cu}$ BHT whilst for Cu-CAT- 1 or $\mathrm{Cu}_{3}(\mathrm{HHTP})_{2}$ the oxidation state is $2+$, and for $\mathrm{Cu}_{3}(\mathrm{HITP})_{2}$ there is a mixture of $\mathrm{Cu}^{\mathrm{I}}$ and $\mathrm{Cu}^{\mathrm{II}}$ ions.

The specific mechanism of charge transport through 2D porous MOF networks is not yet well understood, with some examples exhibiting band-like transport while the behaviour of others fits better with a Mott variable range hopping. Disagreements regarding their electronic structure are also present in 
Table 1 Summary of conductivity and porosity data for the MOGs reported to date

\begin{tabular}{|c|c|c|c|c|c|c|c|}
\hline Material & $\begin{array}{l}\text { Conductivity } \\
{\left[\mathrm{S} \mathrm{cm}^{-1}\right]}\end{array}$ & Measurement method & $\begin{array}{l}\text { Activation } \\
\text { energy }[\mathrm{eV}]\end{array}$ & $\begin{array}{l}\text { Mobility } \\
{\left[\mathrm{cm}^{2} \mathrm{~V}^{-1} \mathrm{~s}^{-1}\right]}\end{array}$ & $\begin{array}{l}\text { Carrier } \\
\text { density }\left[\mathrm{cm}^{3}\right]\end{array}$ & $\operatorname{BET}\left[\mathrm{m}^{2} \mathrm{~g}^{-1}\right]$ & Ref. \\
\hline Со-НHB & $7.3 \times 10^{-8}$ & Pellet, van der Pauw & 0.46 & - & - & 143 & 144 \\
\hline Ni-HIB & 8 & Pellet, van der Pauw & - & - & - & 152 & 142 \\
\hline $\mathrm{Cu}-\mathrm{HIB}$ & 13 & Pellet, van der Pauw & - & - & - & 114 & 142 \\
\hline Co-HIB & 1.67 & Pellet, 4-probe & - & - & - & 240 & 143 \\
\hline Ni-IT & 0.1 & Pellet, van der Pauw & 0.041 & - & - & - & 139 \\
\hline Ni-AT & $3 \times 10^{-6}$ & Pellet, van der Pauw & 0.113 & - & - & - & 140 \\
\hline \multirow[t]{2}{*}{ Ni-BHT } & 0.15 & Pellet, 2-probe & - & - & - & - & 135 \\
\hline & 160 & Flake, van der Pauw & 0.026 & - & - & - & 169 \\
\hline \multirow[t]{2}{*}{$\mathrm{Cu}-\mathrm{BHT}$} & 1580 & Film, 4-probe & 0.00206 & 116 (e)/99 (h) & - & - & 131 \\
\hline & 2500 & Film, 4-probe & - & - & - & - & 145 \\
\hline Pd-BHT & $2.8 \times 10^{-2}$ & Pellet, 4-probe & - & - & - & - & 137 \\
\hline $\mathrm{Ag}-\mathrm{BHT}$ & 250 & Film, 4-probe & 0.4 & - & - & - & 133 \\
\hline $\mathrm{Pt}-\mathrm{BHT} / \mathrm{I}_{2}$ & 0.39 & Pellet, 4-probe & - & - & - & - & 138 \\
\hline $\mathrm{Cu}-\mathrm{HSB}$ & 110 & Pellet, 4-probe & - & - & - & - & 132 \\
\hline \multirow[t]{3}{*}{ Co-CAT-1 } & $2.7 \times 10^{-6}$ & Pellet, 4-probe & - & - & - & 571 & 163 \\
\hline & & & & & & 490 & 130 \\
\hline & $3.3 \times 10^{-3}$ & Film, van der Pauw & - & - & - & - & 187 \\
\hline \multirow[t]{3}{*}{ Ni-CAT-1 } & 0.1 & Pellet, 4-probe & - & - & - & 473 & 163 \\
\hline & & & & & & 425 & 130 \\
\hline & $1.1 \times 10^{-3}$ & Film, van der Pauw & - & - & - & - & 187 \\
\hline \multirow[t]{9}{*}{ Cu-CAT-1 } & 0.2 & Single crystal, 4-probe & - & - & - & - & 163 \\
\hline & $2 \times 10^{-2}$ & Pellet, 4-probe & - & - & - & 284 & 130 \\
\hline & $2 \times 10^{-2}$ & Film, 2-probe & 0.16 & - & - & 540 & 155 \\
\hline & $1 \times 10^{-4}$ & Film, 2-probe & 0.24 & - & - & 348 & 156 \\
\hline & $4.5 \times 10^{-2}$ & Pellet, van der Pauw & 0.15 & - & - & 512 & 157 \\
\hline & 1.5 & Rod, 4-probe & - & - & - & - & 153 \\
\hline & 0.5 & Flake, 2-probe & - & - & - & - & 153 \\
\hline & 0.29 & Film, 4-probe & 0.13 & & & & 160 \\
\hline & 0.21 & Rod, 2-probe & - & - & - & 475 & 188 \\
\hline $\mathrm{Fe}_{3}(\mathrm{HHTP})_{2}$ & $3 \times 10^{-3}$ & Pellet, 4-probe & - & - & - & 69 & 163 \\
\hline $\mathrm{Cu}_{3}(\mathrm{HHTP})(\mathrm{THQ})$ & $2.5 \times 10^{-5}$ & Pellet, 2-probe & 0.3 & & & 441.2 & 161 \\
\hline \multirow[t]{3}{*}{$\mathrm{Ni}_{3}(\mathrm{HITP})_{2}$} & 40 & Film, van der Pauw & - & - & - & 630 & 129 and 164 \\
\hline & 58.5 & Pellet, van der Pauw & - & - & - & - & 189 \\
\hline & 150 & Single crystal, 4-probe & - & - & - & 690 & 153 \\
\hline $\mathrm{Cu}_{3}(\mathrm{HITP})_{2}$ & 0.2 & Pellet, 2-probe & - & - & - & - & 146 \\
\hline \multirow[t]{3}{*}{$\mathrm{Co}_{3}(\mathrm{HTTP})_{2}$} & $1.4 \times 10^{-3}$ & Pellet, van der Pauw & 0.173 & - & - & 370 & 147 \\
\hline & $3.2 \times 10^{-2}$ & Film, van der Pauw & 0.118 & - & - & & \\
\hline & $2.4 \times 10^{-9}$ & Pellet, 4-probe & - & - & - & 266 & 151 \\
\hline $\mathrm{Ni}_{3}(\mathrm{HTTP})_{2}$ & $3.6 \times 10^{-4}$ & Pellet, 4-probe & - & - & - & 166 & 151 \\
\hline $\mathrm{Cu}_{3}(\mathrm{HTTP})_{2}$ & $2.4 \times 10^{-8}$ & Pellet, 4-probe & - & - & - & 171 & 151 \\
\hline $\mathrm{Pt}_{3}(\mathrm{HTTP})_{2}$ & $2.47 \times 10^{-4}$ & Pellet, 2-probe & - & - & - & 329 & 152 \\
\hline $\mathrm{Co}_{3}(\mathrm{TPHS})_{2}$ & $10^{-6}$ & Pellet, 2-probe & 0.294 & - & - & 246 & 148 \\
\hline $\mathrm{Fe}_{3}(\mathrm{THT})_{2}\left(\mathrm{NH}_{4}\right)_{3}$ & $3.4 \times 10^{-2}$ & Film, 4-probe & 0.125 & 230 & $6.2 \times 10^{14}$ & 526 & 171 and 172 \\
\hline Fe-PTC & 10 & Pellet, 4-probe & 0.2 & - & - & - & 174 \\
\hline Cu-HНTX & $8.4 \times 10^{-4}$ & Pellet, 2-probe & 0.21 & - & - & 120 & 175 \\
\hline Cu-DBC & $1 \times 10^{-2}$ & Pellet, 2-probe & 0.3 & - & - & 271 & 176 \\
\hline
\end{tabular}

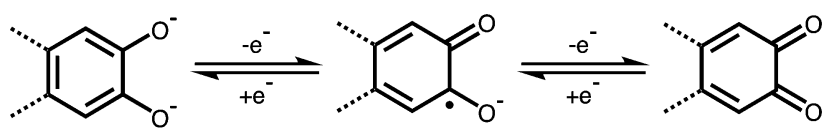

Fig. 8 HHTP is a redox-active linker that can undergo reversible interconversions between catecholate, semiquinonate and quinone forms.

the literature. This is likely due to the different methods used for measuring electrical conductivity and the polycrystalline nature of the samples for variable density of grain boundaries and interparticle resistance. For instance, $\mathrm{Ni}_{3}(\mathrm{HITP})_{2}$ experimentally behaved as a semiconductor when a polycrystalline film was measured by the van der Pauw method. ${ }^{129}$ But, DFT calculations predict a metallic behaviour for the bulk and a narrow bandgap semiconductive one for a single layer. ${ }^{166}$ Further calculations by Foster $e t$ al. attributed the discrepancy to the influence of internal interface defects which introduce transport barriers by breaking the $\pi$-conjugation and disturbing the band structure. ${ }^{167}$ These defects consisting on grain boundaries, strike-slip faults between grains and interlayer displacements, are behind the hopping mechanism that governs charge transport in most MOGs samples. Finally, four-probe measurements of single crystals revealed a bulk metallic behaviour, whilst equivalent pellet measurements of a polycrystalline sample still yielded a semiconductive response. ${ }^{153}$ This is a problem as most MOGs can only be prepared as polycrystalline samples for which intergrain charge hopping is a critical step hindering charge transport. Furthermore, different studies of the same MOG have reported acutely different values depending on the samples' crystallinity, crystallite morphology and the measurement conditions. For example, the conductivity of Cu-CAT-1 varies from $1.5 \mathrm{~S} \mathrm{~cm}^{-1}$ for a single crystal four-probe measurement, ${ }^{153}$ to $10^{-4} \mathrm{~S} \mathrm{~cm}^{-1}$ 
for a $10 \mathrm{~nm}$ thick film on interdigitated electrodes. ${ }^{156}$ Whilst another study found a conductivity one order of magnitude inferior $\left(0.2 \mathrm{~S} \mathrm{~cm}^{-1}\right)$ for a single crystal four-probe measurement. ${ }^{130}$ This highlights another issue in conductive MOFs and CPs: how sample morphology and the technique used for measuring electrical conductivity can have an important influence on the recorded values. $^{168}$

Generally, MOGs with benzene-backbone ligands achieve higher conductivity values than their triphenylene counterparts (see Table 1). Thus, the most conducive MOG is Cu-BHT $(\sigma=$ $2500 \mathrm{~S} \mathrm{~cm}^{-1}$ ), which also holds the record for the most conductive $\mathrm{CP}$ ever reported. ${ }^{145}$ Meanwhile, its selenium equivalent $\mathrm{Cu}-\mathrm{BHS}$ has a lower conductivity of $110 \mathrm{~S} \mathrm{~cm}^{-1} \cdot{ }^{132}$ However, we can consider them special cases of MOG due to their Kagome lattices. The rest of benzene MOGs have slightly lower values, the remaining systems built with the BHT linker are just one of order of magnitude less conductive, like Ni-BHT $\left(\sigma=160 \mathrm{~S} \mathrm{~cm}^{-1}\right)^{169}$ and Ag-BHT $\left(\sigma=250 \mathrm{~S} \mathrm{~cm}^{-1}\right){ }^{133}$ The HIB MOGs are slightly less conductive, but still report high conductivity values for Cu-HIB $\left(\sigma=13 \mathrm{~S} \mathrm{~cm}^{-1}\right){ }^{142} \mathrm{Ni}-\mathrm{HIB}\left(\sigma=8 \mathrm{~S} \mathrm{~cm}^{-1}\right)^{142}$ and Co-HIB $\left(\sigma=1.57 \mathrm{~S} \mathrm{~cm}^{-1}\right) .{ }^{143}$ Finally, Cu-HHB shows a moderate conductivity $\left(\sigma=2.7 \times 10^{-6} \mathrm{~S} \mathrm{~cm}^{-1}\right)$, this highlights the effect of the orbital interaction between the $\mathrm{Cu}^{\mathrm{II}}$ ions and the linkers which is weaker for HHB, likely due to the lower energy of its HOMO in comparison with that of its nitrogen derivative. ${ }^{144}$ Despite having less data available, it is safe to say that triphenylene MOGs present a similar tendency in conductivity values with $\mathrm{Ni}_{3}(\mathrm{HITP})_{2}$ and $\mathrm{Cu}_{3}(\mathrm{HITP})_{2}$ being slightly more conductive than Cu-CAT-1, Co-CAT-1 and Ni-CAT-1. Another factor that influences charge transport in MOGs is interlayer separation. Thus, theoretical studies have pointed that increasing interlayer distances by inserting axial ligands coordinated to the metal ions can lead to changes in the electronic behaviour of these systems. ${ }^{170}$ So far, there is only one example of a MOG with axially coordinated molecules and a single type of layer. $\mathrm{Fe}_{3}(\mathrm{HTTP})_{2}\left(\mathrm{NH}_{4}\right)_{3}$ presents a unique AA inclined stacking with a similar porosity to other triphenylene MOGs $\left(\mathrm{BET}=526 \mathrm{~m}^{2} \mathrm{~g}^{-1}\right)$. The conductivity of the system was extensively studied with time-resolved terahertz spectroscopy, and temperature-dependent four-probe and Hall effect measurements. The $\mathrm{Fe}^{\mathrm{III}}$ MOG was presented as a semiconductor $\left(E_{\mathrm{g}}=0.2 \mathrm{eV}\right)$ with the highest charge carrier mobility reported so far for a MOG $\left(\mu=230 \mathrm{~cm}^{2} \mathrm{~V}^{-1} \mathrm{~s}^{-1}\right) \cdot{ }^{171,172}$

Finally, other MOGs have been obtained using ligands with different connectivity or conjugated core than those with benzene or triphenylene-based linkers. Feng and co-workers recently succeeded in the preparation of a conductive CPs using the linker 1,2,3,4,5,6,7,8,9,10,11,12-perthiolated coronene (PTC), a polycyclic aromatic hydrocarbon with six fused rings and twelve peripheral thiol groups. ${ }^{173}$ In combination with square planar $\mathrm{Fe}^{\mathrm{III}}$ ions, PTC yielded a 2D CP closely related to the MOG family (Fig. 5b). Fe-PTC is constructed by negatively charged planar layers that are vertically stacked in the $\mathrm{AB}$ disposition and have intercalated ammonium cations. The system presents a semiconductive behaviour $(\sigma=$ $10 \mathrm{~S} \mathrm{~cm}^{-1}$ ) and ferromagnetic ordering at low temperature. ${ }^{174}$ Besides, Zhang, Zhao and co-workers used a $C_{3}$-symmetric ligand core of truxene, a popular molecule in organic electronics, functionalized with hydroxy groups. 2,3,7,8,12,13-hexahydroxyl truxene (HHTX) was combined with $\mathrm{Cu}^{\mathrm{II}}$ ions to yield the same hexagonal honeycomb 2D network of MOGs with an eclipsed stacking mode between layers (Fig. 5c). The system presented a semiconductive behaviour as well $\left(\sigma=8.4 \times 10^{-4} \mathrm{~S} \mathrm{~cm}^{-1}\right)$ and a relatively low porosity $\left(\mathrm{BET}=120 \mathrm{~m}^{2} \mathrm{~g}^{-1}\right)$ considering the pore diameter $(2.2 \mathrm{~nm}) .{ }^{175}$ Chen, Ma and collaborators used a $D_{2}$ symmetric redox-active ligand with $\mathrm{Cu}^{\mathrm{II}}$ to prepare $\mathrm{Cu}$-DBC (DBC = dibenzo- $[g, p]$ chrysene-2,3,6,7,10,11,14,15-octaol). This layered MOF is not like other 2D MOFs described here, as it crystallizes into a 4-fold interpenetration of distorted diamond (dia) networks (Fig. 5d). Nevertheless, it displays high electrical conductivity $\left(\sigma=1 \times 10^{-2} \mathrm{~S} \mathrm{~cm}^{-1}\right)$ and moderate porosity $(\mathrm{BET}=$ $\left.271 \mathrm{~m}^{2} \mathrm{~g}^{-1}\right) .{ }^{176}$

2.2.4. Other conductive $2 D$ MOFs. Recently, a new family of conductive 2D phthalocyanine (Pc)-based 2D MOFs has appeared in the literature with three different Pc ligands presented so far (Fig. 5e): 2,3,9,10,16,17,23,24-octa-amino-phthalocyaninato (Pc-NH) ${ }^{177}$ 2,3,9,10,16,17,23,24-octahydroxy-phthalocyaninato $(\mathrm{Pc}-\mathrm{OH})^{178-182}$ and 3,4,12,13,21,22,30,31-octahydroxy-naphthalocyaninato (NPc-OH,). ${ }^{180}$ Various examples have been reported with different metals coordinated to the central cavity and to the terminal groups. All of them are composed of planar layers with square pores that stack in the eclipsed AA-stacking mode. Ni-NiPc-NH has a high conductivity $\left(\sigma=0.2 \mathrm{~S} \mathrm{~cm}^{-1}\right)$ so far for this type of 2D materials, whist the rest are typically one or more orders of magnitude lower. It also shows the highest BET value $\left(593 \mathrm{~m}^{2} \mathrm{~g}^{-1}\right),{ }^{177}$ probably due to the neutral nature of the layers unlike the rest of systems.

Additionally, we can find other conductive 2D frameworks that contain metal ions but are constructed through organic reactions that form strong covalent bonds. Hence, they are considered a subclass of covalent organic frameworks (COFs), known as metal-covalent organic frameworks (MCOFs). ${ }^{183}$ Most conductive MCOFs are based on metallophthalocyanines, ${ }^{184,185}$ but one example $\mathrm{Ni}-\mathrm{COF}$ is composed of $\mathrm{Ni}^{\mathrm{II}}$-salphen units and has a structure reminiscent of MOGs with similar conductivity $\left(\sigma=1.3 \times 10^{-2} \mathrm{~S} \mathrm{~cm}^{-1}\right)$ and BET $\left(362 \mathrm{~m}^{2} \mathrm{~g}^{-1}\right)$ values. $^{186}$

\section{Magnetic bistability in MOFs and CPs}

Magnetism is another fundamental electronic property of MOFs and CPs that contain open-shell metal centres with unpaired electrons. Naturally, it has received significant scientific attention over recent years, ${ }^{190-199}$ especially cooperative magnetic phenomena such as magnetic long-range ordering and spin state switching. Spin crossover (SCO) in particular, is the property with a more extended application in electronic devices $^{25,29,200-205}$ and hence it is the main focus of this review. This phenomenon refers to the transition between high spin (HS) and low spin (LS) electronic configurations that occurs in $\mathrm{d}^{4-7}$ first row transition metal ions coordinated in an octahedral geometry. The spin state is determined by the relative magnitude of the ligand field splitting (the electrostatic field acting at the 


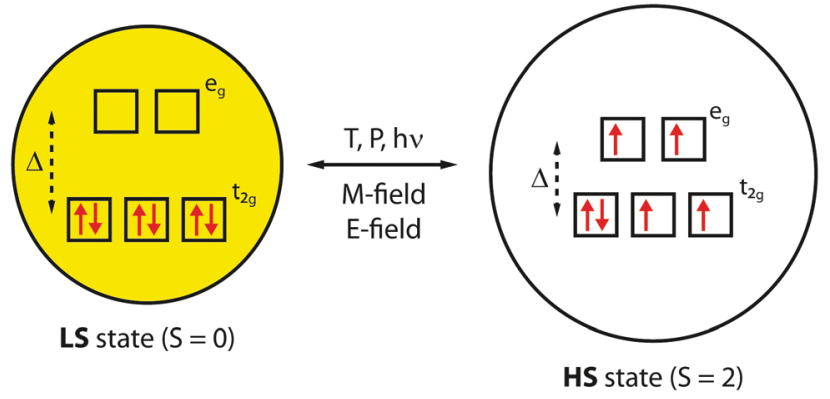

Fig. 9 Scheme depicting the SCO transition between HS and LS configurations for a $d^{6}$ transition metal ion.

central metal ion) compared to the mean spin-pairing energy. If the first is greater, electrons will pair up and completely fill the lower energy $t_{2 g}$ orbitals before populating the higher energy $e_{g}$ orbitals. Hence, the metal ion adopts a diamagnetic LS configuration. If the opposite is true, the unpaired electrons yield a paramagnetic HS state (Fig. 9). Each of the two spin states endows the material with different magnetic, optical, mechanical, electronic and structural properties. ${ }^{206,207}$ The SCO transition between HS and LS can be induced by a variety of external inputs like temperature, light, pressure, guests and magnetic or electric fields. ${ }^{208-212}$ Furthermore, this transition has extremely fast dynamics and can occur cooperatively across the material, thus yielding bulk hysteretic behaviours. ${ }^{213}$ $\mathrm{Fe}^{\mathrm{II}}$ is the most common metal ion to present SCO behaviour followed by $\mathrm{Co}^{\mathrm{II}}$ and $\mathrm{Fe}^{\mathrm{III}},{ }^{214-217}$ whilst $\mathrm{SCO}$ in $\mathrm{d}^{4}$ systems, like $\mathrm{Cr}^{\mathrm{II}}$, $\mathrm{Mn}^{\mathrm{II}}$ and $\mathrm{Mn}^{\mathrm{III}}$, is much less frequent. ${ }^{218}$

\section{1. $\mathrm{Fe}^{\mathrm{II}}$ Hofmann-type coordination polymers}

The perspective of combining the physical properties of metalligand coordination networks (chemical tunability, porosity, processability, etc.) with SCO materials' bistable nature is very attractive from a device point of view. Within the wide variety of 2D and 3D SCO MOFs and CPs that have been reported, $\mathrm{Fe}^{\mathrm{II}}$ Hofmann-type ${ }^{219}$ coordination polymers ( $\mathrm{Fe}^{\mathrm{II}}-\mathrm{HCPS}$ ) are by far the most popular. ${ }^{220}$ Their structure is composed by planar gridlike metal-cyanide layers interconnected by nitrogen-containing ligands (Fig. 10). These sheets alternate octahedral $\mathrm{Fe}^{\mathrm{II}}$ metal ions, responsible for the SCO behaviour, and divalent group 10 metal centres that adopt a square planar geometry. The $\mathrm{Fe}^{\mathrm{II}}$ atoms are coordinated to the nitrogen of four cyanide groups and to two additional pillaring ligands. If these are bis-monodentate, the resulting structure is generally $3 \mathrm{D}$, with a general formula of $\left[\mathrm{Fe}^{\mathrm{I}}(\mathrm{L})\left\{\mathrm{M}^{\mathrm{II}}(\mathrm{CN})_{4}\right\}\right]\left(\mathrm{L}=\right.$ ligand, $\mathrm{M}^{\mathrm{II}}=\mathrm{Ni}^{\mathrm{II}}, \mathrm{Pd}^{\mathrm{II}}$ or $\left.\mathrm{Pt}^{\mathrm{II}}\right)$, as a single pillaring ligand covalently bonds two adjacent layers (Fig. 10b). On the other hand, when monodentate ligands (usually py derivatives) are used, a 2D network is obtained with a general formula of $\left[\mathrm{Fe}^{\mathrm{I}}(\mathrm{L})_{2}\left\{\mathrm{M}^{\mathrm{II}}(\mathrm{CN})_{4}\right\}\right]$, although neighbouring sheets are still interconnected via $\pi-\pi$ interactions (Fig. 10c). $3 \mathrm{D} \mathrm{Fe}{ }^{\mathrm{II}}-\mathrm{HCPs}$ networks usually have permanent porosity, which can be used to influence the SCO transition through the incorporation of guests that affect the ligand field splitting of $\mathrm{Fe}^{\mathrm{II}} .{ }^{221,222}$ Real, Bousseksou, Kitagawa and collaborators have extensively investigated this possibility. Thus using either invasive guests to induce redox changes in the network, ${ }^{223}$ or softer guests that more subtly modify the coordination environment through electronic interactions with the pillaring linkers ${ }^{224-235}$ or by blocking the rotation of their aromatic rings. ${ }^{236-238}$ Sometimes, this can trigger the appearance of multi-step spin transitions by breaking the symmetry of the Fe ${ }^{\mathrm{II}}-\mathrm{HCP}$ network. ${ }^{228,231-235}$ The stabilization of either the HS or the LS state can occur depending on the chemical nature, which determines the type and strength of electronic interactions, and the size of the guest molecules, which delimits the expansion/contraction of the network characteristic of the SCO transition. ${ }^{237,239,240}$ Conversely, in $2 \mathrm{D} \mathrm{Fe}{ }^{\mathrm{II}}$-HCPs the interdigitated arrangement of the consecutive layers does not leave any pore space for regular permanent guest sorption. Moreover, they usually present lower SCO transition temperatures and narrower hysteresis loops due to a lower degree of cooperativity in-between the non-covalently bonded layers. However, a few examples prepared by Neville, Kepert and collaborators escape this tendency by using functionalised 1,2,4triazoles as axial $\mathrm{Fe}^{\mathrm{II}}$ ligands (Fig. 10d). Hence, they report near ambient transition temperatures and guest dependent SCO properties, as guest solvents interact with the free $\mathrm{N}$ of the triazole ring. ${ }^{241-243}$ Additionally, some other $2 \mathrm{D}$ Fe ${ }^{\mathrm{II}}$-HCPs with py derivatives as pillaring linkers also present guest sorption capabilities when the number of layers is reduced below a critical number. In this sense, $\left[\mathrm{Fe}^{\mathrm{I}}\left(\mathrm{L}^{\prime}\right)_{2}\left\{\mathrm{Pt}(\mathrm{CN})_{4}\right\}\right]\left(\mathrm{L}^{\prime}=\right.$ py, pyrimidine and isoquinoline) ultrathin a)

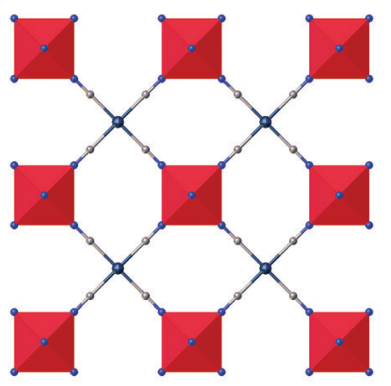

b)

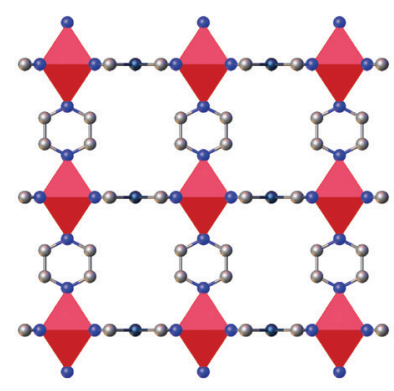

c)

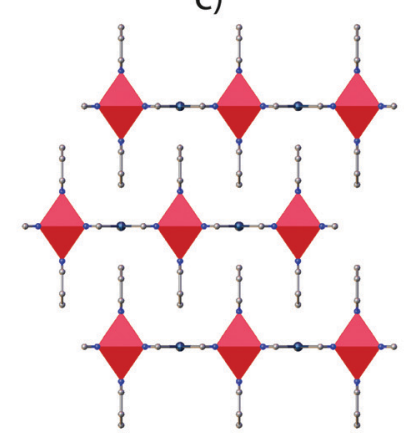

d)

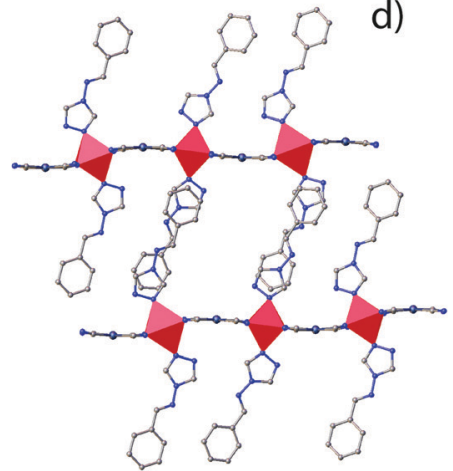

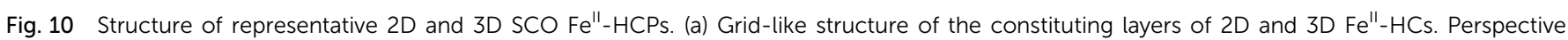

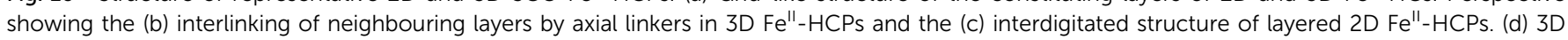
structure of $\mathrm{Fe}^{\mathrm{II}}$-HCPs built from functionalised 1,2,4-triazoles. 
films ( $<30$ layers) have been proven capable to accommodate solvents in-between their layers, through an axial lattice expansion observed via synchrotron XRD. ${ }^{244-246}$

\subsection{Electrical conductivity and SCO}

The combination of SCO and electrical conductivity in a single material has been a long-desired goal for material scientists. Particularly, in the context of the use of electrical stimuli to control the SCO transition or vice versa, as electrical current would constitute a more feasible way of managing input/output signals in a SCO device setting rather than temperature or pressure. ${ }^{203}$ Unfortunately, most SCO compounds known to date are insulating, particularly SCO CPs and MOFs. This is still a major obstacle for their integration in electronic devices.

Looking at the present state of the area, the main goal of most researchers in the SCO field has exclusively centred around the transition phenomena itself. In the meantime, the challenge of producing highly conductive SCO materials has been largely overlooked. Some efforts have been dedicated to produce materials that display SCO together with high electrical conductivity values. However, only one of the previously described strategies for infusing electrical conductivity in non-magnetic MOFs and CPs has been really explored. Following the path of molecular conductors, the strategy of including electroactive TTF moieties in the organic linkers has been attempted to induce electrical conductivity into $\mathrm{Fe}^{\mathrm{II}}$-HCPs and other SCO CPs. For $\mathrm{Fe}^{\mathrm{II}}$-HCPs, the axial organic ligand that connects the metalcyanide layers can be chemically modified to incorporate an electroactive group. Real and collaborators used this strategy to prepare $\left\{\mathrm{Fe}(\mathrm{ttf}-\mathrm{adpy})_{2}\left[\mathrm{M}(\mathrm{CN})_{4}\right]\right\} \cdot n \mathrm{H}_{2} \mathrm{O}$ (ttf-adpy $=4$-tetrathiofulvalenylcarboxamidopyridine; $\mathrm{M}=\mathrm{Ni}, \mathrm{Pd}, \mathrm{Pt}$ ), a series of $2 \mathrm{D}$ $\mathrm{Fe}^{\mathrm{II}}$-HCPs ${ }^{247}$ Here, $\mathrm{Fe}^{\mathrm{II}}$ centres are axially coordinated to ttf-adpy ligand, which consist of a pyridine unit bonded to a TTF core via a secondary amide. Electrochemical studies indicated that free ttf-adpy has low oxidation potential to form the radical. As result, the ttf-adpy units were neutral for all cases and the $2 \mathrm{D} \mathrm{Fe}$-HCPs displayed low cooperative SCO transitions strongly dependent on their water content. ${ }^{247}$ As commented in Section 2, Wang et al. prepared $\left\{[\mathrm{Fe}(\mathrm{dca})]\left[\mathrm{TTF}-(\mathrm{py})_{4}\right] \cdot \mathrm{ClO}_{4} \cdot \mathrm{CH}_{2} \mathrm{Cl}_{2} \cdot 2 \mathrm{CH}_{3} \mathrm{OH}\right\}_{n}$, a noninterpenetrated $\mathrm{CP}$ with open pore channels and octahedrally $\mathrm{N}$-coordinated $\mathrm{Fe}^{\mathrm{II}}$ ions that undergoes gradual and incomplete SCO in its as-synthesized form. ${ }^{73}$ When a single crystal was exposed to a $\mathrm{I}_{2}$ solution, $\left\{[\mathrm{Fe}(\mathrm{dca})]\left[\mathrm{TTF}(\mathrm{py})_{4}\right] \cdot 0.5 \mathrm{I}_{3} \cdot \mathrm{ClO}_{4} \cdot \mathrm{CH}_{2} \mathrm{Cl}_{2}\right.$. $\left.\mathrm{CH}_{3} \mathrm{OH} \cdot \mathrm{C}_{6} \mathrm{H}_{12}\right\}_{n}$ was obtained, which has $\mathrm{I}_{3}{ }^{-}$anions in between oxidized TTF $^{*+}$ radical ligands (Fig. 11a). Surprisingly, this compound did maintain the SCO transition, although it was significantly more incomplete and more gradual (Fig. 11b). ${ }^{73}$ These being the only published attempts at making a highly conductive SCO framework material, reinforces the idea that a lot more effort needs to be dedicated to this challenging task. Nevertheless, we can find one additional example in the 1D chain $\mathrm{CP}\left[\mathrm{Co}(\mathrm{MQ})_{2}\right](\mathrm{MQ}=8$-mercaptoquinoline), a semiconductor $(\sigma=$ $\left.1.7 \times 10^{-7} \mathrm{~S} \mathrm{~cm}^{-1}\right)$ with a gradual SCO transition of the $\mathrm{Co}^{\mathrm{II}}$ ions in an unusual coordination environment of nitrogen and sulphur atoms. ${ }^{248}$ Also, in a somewhat unique strategy, Martin and Lemaire, prepared a SCO conducting metallopolymer based a)
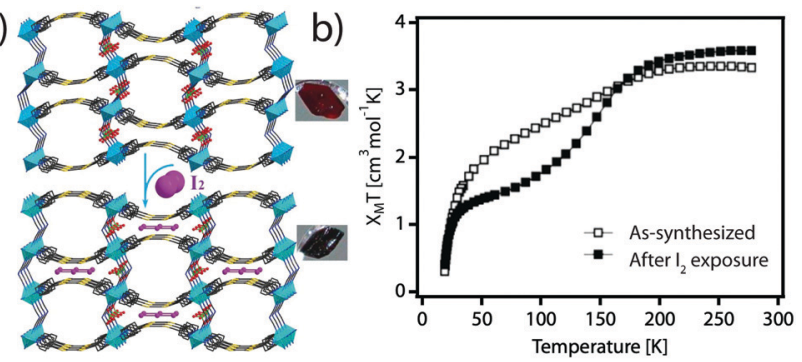

Fig. 11 (a) View along the $c$ axis of as-synthesized $\left\{[\mathrm{Fe}(\mathrm{dca})]\left[T T \mathrm{~T}-(\mathrm{py})_{4}\right]\right.$. $\left.\mathrm{ClO}_{4} \cdot \mathrm{CH}_{2} \mathrm{Cl}_{2} \cdot 2 \mathrm{CH}_{3} \mathrm{OH}\right\}_{n}$ and $\left\{[\mathrm{Fe}(\mathrm{dca})]\left[\mathrm{TTF}(\mathrm{py})_{4}\right] \cdot 0.5 \mathrm{I}_{3} \cdot \mathrm{ClO}_{4} \cdot \mathrm{CH}_{2} \mathrm{Cl}_{2} \cdot \mathrm{CH}_{3} \mathrm{OH}\right.$. $\left.\mathrm{C}_{6} \mathrm{H}_{12}\right\}_{n}$ after of $\mathrm{I}_{2}$ exposure. Next to the structures photographs of the corresponding crystals are shown. (b) Temperature dependence of the molar magnetic susceptibilities before and after of $\mathrm{I}_{2}$ exposure. Reproduced from ref. 73 with permission from Wiley, copyright 2017.

on $\mathrm{N}-(8$-quinolyl)salicylaldimine type ligand covalently linked to a thiophene polymer. The electropolymerized film showed a gradual SCO transition with no thermal hysteresis but it did show hysteretic conductivity with temperature. Although the conductivity values are the highest so far for a SCO material (approximately 10-100 $\mathrm{S} \mathrm{cm}^{-1}$ ), the relationship between SCO and conductivity was not clearly demonstrated. ${ }^{249}$

The rest of the studies of charge transport properties of SCO materials have dealt with compounds that were not specifically designed with conductivity in mind and are therefore poor conductors. Again, there is not a very extensive amount of work done concerning this type of materials, particularly SCO CPs and MOFs, as it is extremely challenging to measure current values below the detection limit, especially at low temperatures. In a recent publication, Martí-Gastaldo, Tatay and co-workers fabricated ultrathin films (thickness $<15 \mathrm{~nm}$ ) to analyse the conductivity of $2 \mathrm{D} \mathrm{Fe} \mathrm{Fe}^{\mathrm{II}}$-HCPs: $\left[\mathrm{Fe}(\mathrm{L})_{2}\left\{\mathrm{Pt}(\mathrm{CN})_{4}\right\}\right]\left(\mathrm{L}=\right.$ py, pyrimidine, and isoquinoline). ${ }^{246}$ Out-ofplane charge transport was then measured using liquid Eutectic Gallium Indium alloy (EGaIn) as top electrode (Fig. 12). Analysis of thickness dependent current density measurements at room temperature (HS) yielded a low decay coefficients $\left(\beta=0.03-0.08 \AA^{-1}\right)$, characteristic of a hopping regime $\left(<0.1 \AA^{-1}\right)$ and in line with other SCO ultrathin films measured in the same conditions. ${ }^{250,251}$ Thus, in this through-space hopping conduction, the axial linker L heavily influences the vertical charge transport by altering the interlayer distance, and also governs the electronic interaction with the EGaIn top contact. ${ }^{246}$

Finally, the only other SCO CP whose charge transport properties have been broadly examined is $\left[\mathrm{Fe}(\mathrm{Htrz})_{2}(\mathrm{trz})\right]\left(\mathrm{BF}_{4}\right)$ (Htrz $=1,2,4-1 H$-triazole), an ionic 1D CP formed by chains of $\mathrm{Fe}^{\mathrm{II}}$ centres linked by triazoles ${ }^{252,253}$ that has a hysteretic SCO transition at around room temperature. ${ }^{254}$ Bousseksou, Salmon and co-workers performed most of the work with this material (Fig. 13). ${ }^{255-260}$ These different studies were performed with different set-ups (two-probe contacts or interdigitated electrodes, ${ }^{259,260} \mathrm{DC}^{258}$ or AC conductivities $\left.{ }^{255,257,261}\right)$ and using samples with slightly different crystallite sizes $(0.7-5 \mu \mathrm{m} \times 200-300 \mathrm{~nm}$ high-aspect ratio needles $^{258-260}$ or $\sim 200 \mathrm{~nm}$ nearly spherical particles ${ }^{255,257,258,261}$ ) and large electrode gaps of several micrometres. Nonetheless, most of them yielded comparable results and pointed to the same 

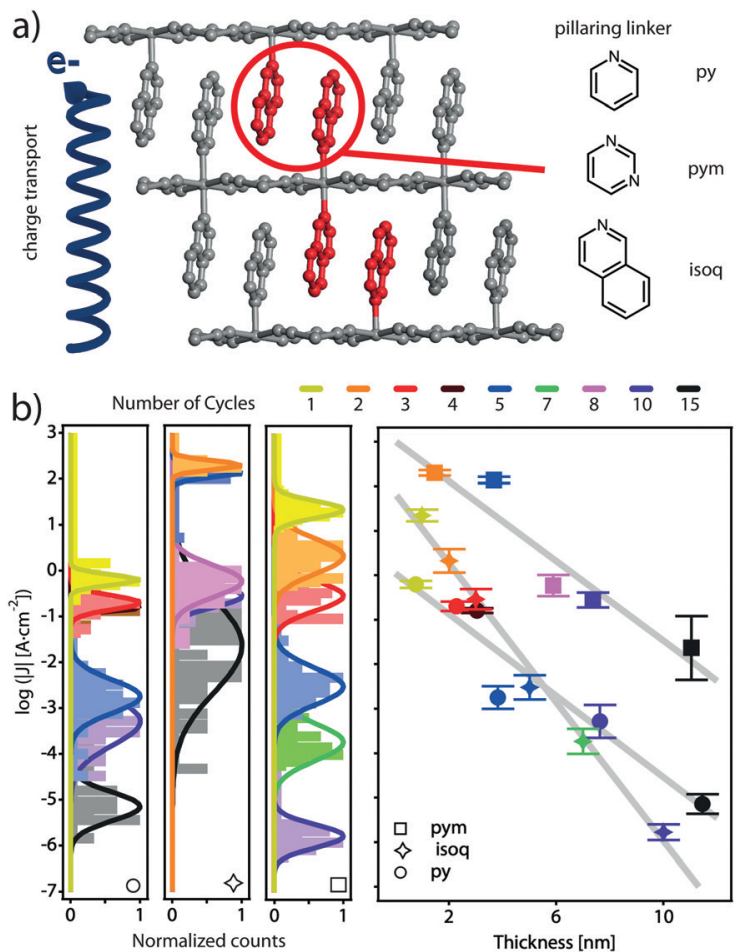

Fig. 12 (a) Schematic crystallographic structure of the $\left[\mathrm{Fe}(\mathrm{L})_{2}\left\{\mathrm{Pt}(\mathrm{CN})_{4}\right\}\right]$ $[L=$ pillaring linker, pyridine (py), pyrimidine (pym), or isoquinoline (isoq)] 2D Fe"-HCPs used in by Martí-Gastaldo and co-workers. (b) Dependence of the logarithm of the absolute value of the current-density $(\log |J|)$ measured at $0.1 \mathrm{~V}$ as a function of film thickness films. Maximum number of counts for each histogram has been normalized to 1 . Solid lines are Gaussian fits to the data. Error bars correspond to the Gaussian width. Straight dashed lines are a linear fit to the data. In all cases, colours indicate the number of growth cycles. Reproduced from ref. 246 with permission from the American Chemical Society, copyright 2019.

conclusion: the HS form is less conductive than the LS state (Fig. 13b). However, there was one sample of spherical NPs that showed the opposite trend, a more conductive HS phase. The authors ascribed this exception to small changes in the defects of the structure or in the particle morphology (grain boundary effects). ${ }^{258}$ Surprisingly, the magnetic measurements on this particular sample showed similar SCO properties to the others. Precise conductivity values were only given in one publication for DC measurements on pellets of needle-like micrometric crystallites $\left(\sigma_{\mathrm{HS}}=5 \times 10^{-1} \mathrm{~S} \mathrm{~cm}^{-1} ; \sigma_{\mathrm{LS}}=6 \times 10^{-4} \mathrm{~S} \mathrm{~cm}^{-1}\right)$ and spherical nanoparticles $\left(\sigma_{\mathrm{HS}}=9 \times 10^{-7} \mathrm{~S} \mathrm{~cm}^{-1} ; \sigma_{\mathrm{LS}}=6 \times 10^{-7} \mathrm{~S} \mathrm{~cm}^{-1}\right) .{ }^{258}$ Again, the difference between samples is quite remarkable, especially considering that they both displayed similar SCO properties. Increased grain boundaries are probably to blame for this disparity.

A different work by Dugay, Coronado and co-workers reported similar results for high-aspect ratio nanorods $(10 \times 25$ and $6 \times$ $44 \mathrm{~nm}$ in size) deposited onto interdigitated electrodes with a gap of $50 \mathrm{~nm} .^{262}$ The LS state was found to be the highconduction state by up to two orders of magnitude difference and a hysteresis loop of $45 \mathrm{~K}$ was observed in the electrical current versus temperature plot. But, as the reproducibility of the
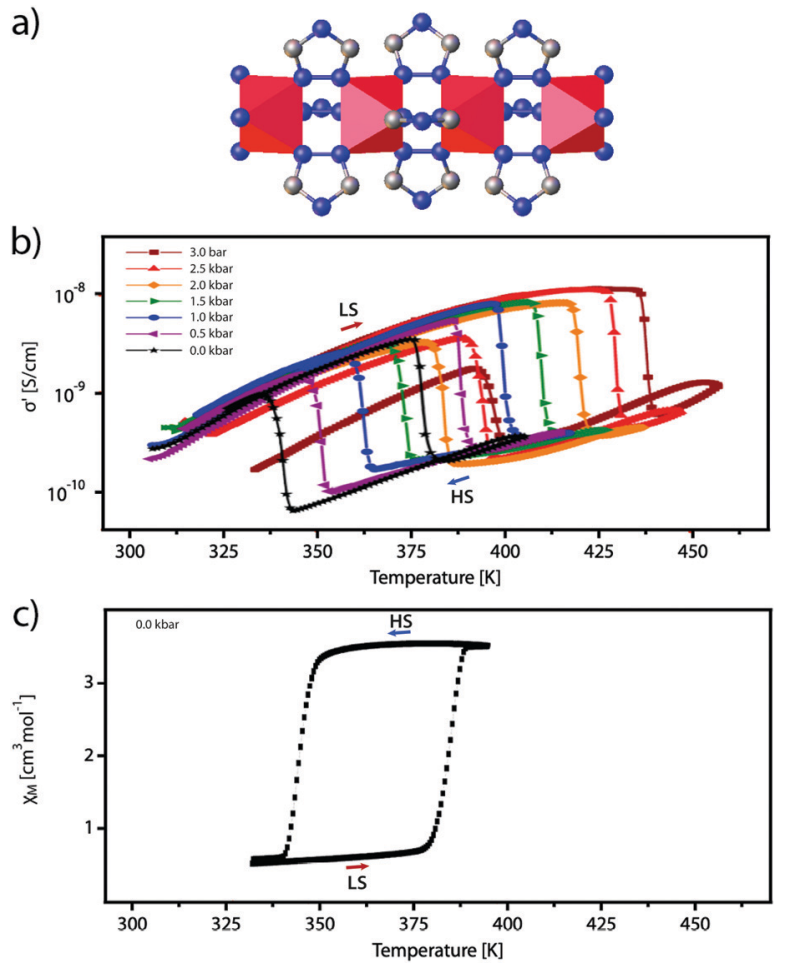

Fig. 13 (a) Schematic crystallographic structure of $\left[\mathrm{Fe}(\mathrm{Htrz})_{2}(\operatorname{trz})\right]\left(\mathrm{BF}_{4}\right)$, anions have been omitted for clarity. (b) Temperature dependence of the real part of the $\mathrm{AC}$ conductivity recorded at $10 \mathrm{kHz}$ at various applied pressures. (c) Temperature dependence of the molar magnetic susceptibility. Adapted from ref. 261 with permission from the American Chemical Society, copyright 2017.

measurements was analysed, they were found not to be reversible after only one heating/cooling cycle. Current values progressively decreased below the detection limit, presumably as more $\mathrm{Fe}^{\mathrm{II}}$ centres get trapped in the HS configuration. Later, they managed to conduct a more reproducible study of $110 \times 50 \mathrm{~nm}$ core-shell $\mathrm{NPs}$ of $\left[\mathrm{Fe}(\mathrm{Htrz})_{2}(\mathrm{trz})\right]\left(\mathrm{BF}_{4}\right)$ with a $11 \mathrm{~nm} \mathrm{SiO}{ }_{2}$ shell, which were contacted by graphene single layer electrodes with a gap of $300 \mathrm{~nm}$. Results showed again a more conductive LS state. ${ }^{263}$ Bousseksou, Salmon, Rotary and co-workers also argued that the charge transport in big particles of $\left[\mathrm{Fe}(\mathrm{Htrz})_{2}(\mathrm{trz})\right]\left(\mathrm{BF}_{4}\right)$ occurs via polaron hopping. Hence, they associate the higher conductivity in the LS state to its higher stiffness (i.e., higher phonon frequencies), which results in higher hopping rates. ${ }^{257}$ However, in a separate study, Dugay, Coronado and co-workers used time-resolved microwave conductivity to show that there is actually two different transport regimes in the LS state: tunnelling through shallow traps which transits into a trap-free hopping regime with increasing temperature. ${ }^{264}$

All the above studies of $\left[\mathrm{Fe}(\mathrm{Htrz})_{2}(\mathrm{trz})\right]\left(\mathrm{BF}_{4}\right)$ show an opposite trend to the results reported by Prins et al., which reported that the HS state has a larger conductivity than its counterpart. Nanoparticles of $11 \pm 5 \mathrm{~nm}$ were placed between gold electrodes with a gap of either $100 \mathrm{~nm}$ or $1 \mu \mathrm{m}$, thus operating in the tunnelling regime. ${ }^{265}$ The authors suggested that the increase in Fe-N bond lengths $(0.2 \AA)$ upon the LS to HS spin transition could lower the tunnel barrier, thus leading to an increase of the conductance. This reinforces the 
premise that different charge transport regimes (tunnelling versus hopping) yield a different predominant spin state in terms of electrical conductivity. Interestingly, a hysteresis loop of similar width to the magnetic curve was obtained in the conductance as a function of temperature plot. However, concerning reproducibility: out of ten samples, the conductance switch occurred in $50 \%$ of them, while just $30 \%$ displayed hysteresis and in the remaining $20 \%$ the change in conductance was (partially) irreversible. ${ }^{265}$

However, there is one conflicting study to this idea concerning $\left[\mathrm{Fe}(\mathrm{Htrz})_{2}(\mathrm{trz})\right]\left(\mathrm{BF}_{4}\right)$. Torres-Cavanillas et al. prepared core@shell spherical NPs with a Au core $(c a .12 \mathrm{~nm})$ and a $\left[\mathrm{Fe}(\mathrm{Htrz})_{2}(\mathrm{trz})\right]\left(\mathrm{BF}_{4}\right)$ shell $(4 \mathrm{~nm}) .{ }^{266}$ Charge transport measurements were then performed in a device fabricated by depositing the NPs onto interdigitated electrodes. The device showed a sharp hysteretic transition in thermal variation of the electrical current with an on/off ratio of 1500 between a high conductance LS and a low conductance HS state. This conductance transition was explained via a percolation model. $\left[\mathrm{Fe}(\mathrm{Htrz})_{2}(\operatorname{trz})\right]\left(\mathrm{BF}_{4}\right)$ NPs of $16 \mathrm{~nm}$ without the Au core were also prepared, but they were too insulating for transport measurements. ${ }^{266}$ Again, previous measurements of small NPs $(11 \pm 5 \mathrm{~nm})$ showed opposite results for the HS/LS conductivity change. ${ }^{265}$

\subsection{Electrical properties of individual spin states}

Regardless of achieving materials that show both SCO transition and high conductivity, gathering information on the electrical properties of each of the individual spin states is also of vital importance for device integration. However, the amount of work done in this sense is still scarce with very few studies of bulk materials, nanometric particles or films of extended CPs. The vast majority of them being materials composed of discrete molecular complexes and not framework systems that are the main scope of this review. At the present time, there is not even clear evidence to draw definitive general conclusions about which spin state is more conductive (HS or LS), due to contradicting experimental results. This is a difficult problem to tackle as most SCO materials have already low charge carrier mobilities at room temperature, so cooling them down yields negligible current values. Hence, it is extremely challenging to obtain reliable measurements in bulk samples or to fabricate viable nanometric devices. Thus far, the options have been restricted to either working with SCO systems with HS-to-LS transitions that occur at high temperatures, reducing the electrode separation or downsizing particles or film-based devices in order to operate in the tunnelling regime. But of course, modifying the charge transport mechanism from hopping to tunnelling can also influence the device resistance variation with the spin state, and so can the SCO/electrode interface. All this along with the already challenging fabrication and characterization of devices as well as reproducibility issues, add to the difficulty in elucidating the relationship between spin state and conductivity. ${ }^{203,204}$ Most reports that observe a more conductive HS configuration are of single molecule, monolayer or few-layer junctions (tunnelling regime), while most of the papers pointing in the opposite direction are of large contact are devices or nanocrystalline samples (hopping regime). Nonetheless, there are a few exceptions to this classification. ${ }^{266-270}$ So far there is only one record of a single crystal measurement with the two-probe method. ${ }^{271}$ Undoubtedly, the synthetic difficulties in producing large crystals are also an important obstacle for SCO materials along with their low charge carrier mobilities.

\subsection{Electrical conductivity and valence tautomerism in PBAs}

Valence tautomerism (VT) can be considered another type of cooperative magnetic bistability, as it consists on the reversible interconversion between redox states of different metal ions in polynuclear coordination compounds. We find a similar case to the conductivity and SCO relationship in the conductivity dependence with VT switching that can be observed in some PBAs. As previously mentioned in Section 2.2.2, PBAs are analogues of $\mathrm{Fe}_{4}^{\mathrm{III}}\left[\mathrm{Fe}^{\mathrm{II}}(\mathrm{CN})_{6}\right]_{3} \cdot n \mathrm{H}_{2} \mathrm{O}$ with a general formula of $\mathrm{A}_{x} \mathrm{M}_{y}\left[\mathrm{M}(\mathrm{CN})_{6}\right]_{z} \cdot n \mathrm{H}_{2} \mathrm{O}$, in which $\mathrm{A}$ is an alkali metal ion and $\mathrm{M}$ are transition metal ions. ${ }^{111}$ The octahedral coordination of the transition metal ions with cyanide anions results in cubic (Fig. 14a), monoclinic or rhombohedral 3D networks depending on the distortion of the coordination sphere. If the metalcyanide network is not neutral, the pores are occupied by alkali counterions. Usually, the transition metal ion coordinated with the nitrogen of the cyanide ligand presents a trivalent oxidation state in the HS configuration and the one coordinated with the carbon presents a LS divalent oxidation state. The process in which an electron can travel in-between these metal centers can be classified as VT. FeCo PBAs are widely known to present VT, also referred to as charge-transfer-induced spin transition (CTIST), ${ }^{273,274}$ due to the reversible transition between diamagnetic $\mathrm{Fe}^{\mathrm{II}-\mathrm{LS}}-\mathrm{CN}-\mathrm{Co}^{\mathrm{III}-\mathrm{LS}}$ and paramagnetic $\mathrm{Fe}^{\mathrm{III}-\mathrm{LS}}-\mathrm{CN}-\mathrm{Co}^{\mathrm{II}-\mathrm{HS}}$ configurations. ${ }^{275}$ Similarly to SCO, the CTIST transition can be triggered by temperature or light irradiation and provokes changes in the compounds' bond lengths, magnetic, photochromic and electric properties. $^{276}$

The first report on the dependence between electrical conductivity and VT was published by Sato et al. for the FeCo PBAs $\mathrm{Na}_{0.5} \mathrm{Co}_{1.25}\left[\mathrm{Fe}(\mathrm{CN})_{6}\right] \cdot 4.8 \mathrm{H}_{2} \mathrm{O}$ and $\mathrm{Na}_{0.38} \mathrm{Co}_{1.31}\left[\mathrm{Fe}(\mathrm{CN})_{6}\right] \cdot 5.4 \mathrm{H}_{2} \mathrm{O}^{272}$ These compounds show an abrupt change in conductivity at the same temperature of the magnetic phase transition between $\mathrm{Fe}^{\mathrm{II}-\mathrm{LS}}$ $\mathrm{CN}-\mathrm{Co}^{\mathrm{III}-\mathrm{LS}}$ and $\mathrm{Fe}^{\mathrm{III}-\mathrm{LS}}-\mathrm{CN}-\mathrm{Co}^{\mathrm{II}-\mathrm{HS}}$ with a similar hysteresis loop in both of them (Fig. 14). Moreover, the conductivity phase can also be altered by applying an electric field. This behaviour has also been observed for MnFe PBAs. Thus, Molnár et al. observed a similar

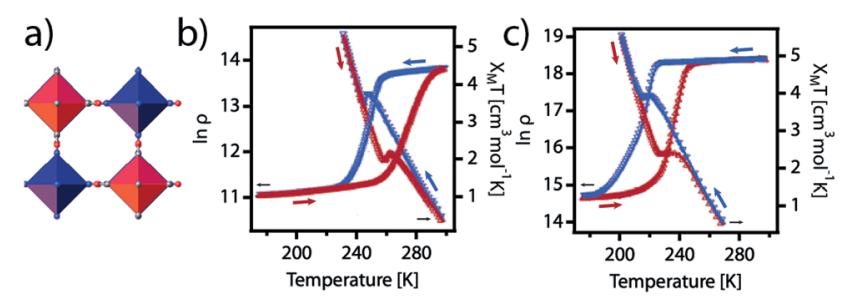

Fig. 14 (a) Schematic crystallographic structure of FeCo PBA. The vacancies at the $\left[\mathrm{Fe}(\mathrm{CN})_{6}\right]$ site and the $\mathrm{Na}^{+}$atoms have been omitted for clarity. Temperature dependence of resistivity and magnetization for (b) $\mathrm{Na}_{0.5} \mathrm{Co}$ ${ }_{1.25}^{\prime \prime}\left[\mathrm{Fe}^{\prime \prime \prime}(\mathrm{CN})_{6}\right] \cdot 4.8 \mathrm{H}_{2} \mathrm{O}$ and (c) $\mathrm{Na}_{0.38} \mathrm{CO}_{1.31}^{\prime \prime}\left[\mathrm{Fe}^{\text {III }}(\mathrm{CN})_{6}\right] \cdot 5.4 \mathrm{H}_{2} \mathrm{O}$. Adapted from ref. 272 with permission from the American Chemical Society, copyright 2004. 
effect in a series of $\mathrm{Rb}_{x} \mathrm{Mn}\left[\mathrm{Fe}(\mathrm{CN})_{6}\right] y \cdot z \mathrm{H}_{2} \mathrm{O}$ PBAs. ${ }^{277,278}$ Additionally electrical conductivity modulation with VT has been observed in cyanide-bridged CPs of lower dimensionality than 3D PBAs. For instance, the $1 \mathrm{D}$ chain $\left\{(\mathrm{Tp}) \mathrm{Fe}(\mathrm{CN})_{3} \mathrm{Co}((R)-\mathrm{pabn})\left(\mathrm{BF}_{4}\right)\right\} \cdot \mathrm{H}_{2} \mathrm{O}(\mathrm{Tp}=$ hydrotris(pyrazol-1-yl)borate; $(R)$-pabn $=(R)-N(2), N\left(2^{\prime}\right)$-bis(pyridine2-ylmethyl)-1,1'-binaphtyl-2,2'-diamine) shows and evolution from insulator to semiconductor with the CTIST transition. ${ }^{279}$

\section{Nanostructuration strategies for device fabrication}

All the strategies reviewed above highlight the wide range of possibilities to produce electrically active and magnetically bistable CPs and MOFs. This potential for tailor-made materials has pushed industrial and academic researchers to initiate the path to integrate them in electronic devices, resulting in several reviews and roadmaps that indicate the current challenges and technological barriers. ${ }^{23-25,57,58}$ Nevertheless, before truly considering them as components of an electronic device compatible with CMOS technology, it is necessary to understand how these materials function within the device and how they interface with it. On this order of ideas, it is first necessary to deposit and study these materials on surfaces as thin films. They have to be carefully processed with exquisite control over several factors that play an important role in the device performance. Ideally, film thickness, substrate coverage, homogeneity, roughness, crystallinity and crystalline orientation with respect to the substrate have to be carefully controlled to achieve a viable device. Controlling the above parameters is necessary so that the designer properties of the original bulk material are equivalent in its nanostructured form. The quality of the film can be drastically changed depending on the deposition method, severely affecting material performance. Besides, this structuration also presents a challenge for chemical and physical characterization. ${ }^{27}$ In the following sections, we will review some of the techniques used for the deposition of CPs and MOFs as thin films.

As shown in Fig. 15, we will divide deposition methods depending on how the final material is formed: (i) deposition from crystals or colloids/colloidal dispersions, in which materials are previously synthesized and then deposited on the surface, (ii) direct synthesis of micrometer-thick films that are generally grown by seeding methods and (iii) layer-by-layer (LbL) films grown by sequential deposition methods. As we will see later, some of those methods can be improved by functionalizing substrates to promote and direct the nucleation, orientation, and structure of the resulting film.

\subsection{Deposition of pre-formed materials}

This type of procedure is composed of two steps. The first one involves the synthesis of the desired material in solution

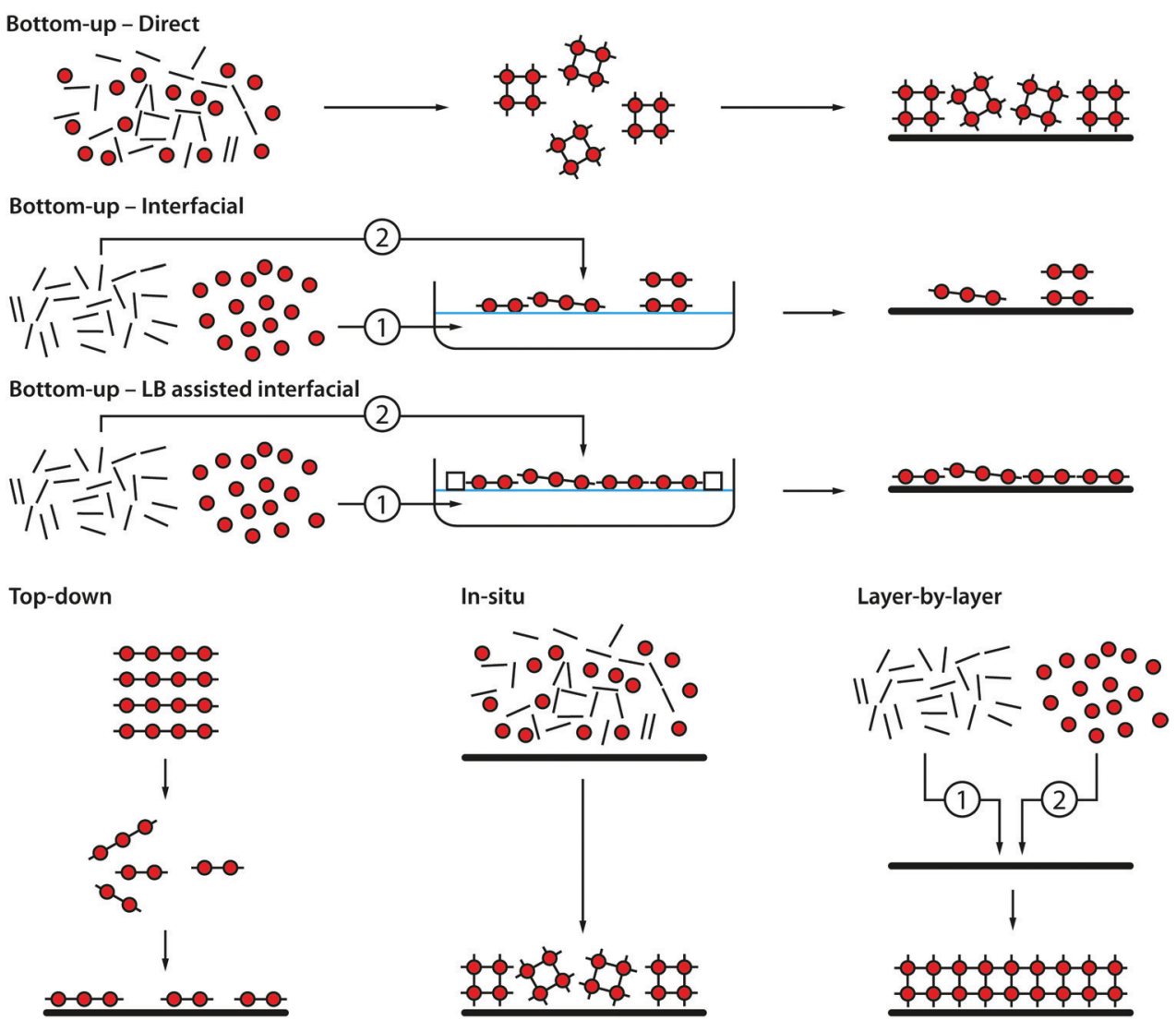

Fig. 15 Schematic illustrations exemplifying the most common protocols used for the synthesis and/or transfer of thin films of MOFs and CPs to solid substrates. 
a)
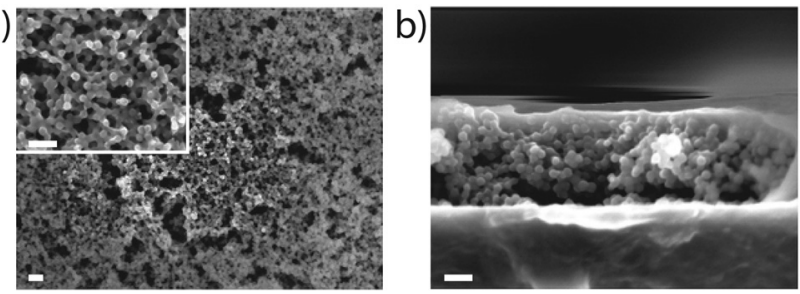

Fig. 16 Field emission SEM images of thin films prepared by dip-coating a silicon substrate in a colloidal solution of ZIF- 8 NPs. (a) Top view and (b) side view. Scale bar length is $100 \mathrm{~nm}$. Reproduced from ref. 282 with permission from the Royal Society of Chemistry.

(bottom-up approach) or as bulk material that in turn can be physically or liquid-phase delaminated (top-down approach). In the second step, the pre-formed material is deposited on the surface.

4.1.1. Bottom-up deposition. Fabrication of MOF films by this method has been used, among others, by Sanchez, Serre, and co-workers. It consists on preparing MOF particles with welldefined size and transferring them onto silicon substrates by dip-coating. They experimentally demonstrated this concept with three different MOF structures: MIL-89 $\left[\mathrm{Fe}_{6} \mathrm{O}_{2} \mathrm{Cl}_{2}(\mathrm{BDC})_{6}\right],{ }^{280}$ MIL-101 $\left[\mathrm{Cr}_{3} \mathrm{OF}(\mathrm{BDC})_{2}\left(\mathrm{H}_{2} \mathrm{O}\right)_{2}\right]^{281}$ and ZIF-8 $\left[\mathrm{Zn}^{2}\left(\mathrm{Cu}_{4} \mathrm{H}_{5} \mathrm{~N}_{2}\right)_{2}\right]$ (Fig. 16). ${ }^{282}$ Depending on the concentration of the particles in solution, 2-3 layers of particles were deposited at the same time. Repetition of the dip-coating process led to increasingly thicker films. However, the mechanical resistance of such films is moderate due to their poor adhesion to the substrate. The advantage of this method is that the size and crystallinity of the particles is controlled in a first step and is not limited by the preparation of the films. On the other hand, it relies on the preparation of stable colloidal dispersions and does not permit producing oriented films. These films can broadly be described as bulk crystalline powders resting on a substrate due to the high roughnesses of the films and their irregular micrometric thicknesses.

Better control on film thickness and orientation can be achieved when the formation of the material to deposit takes place at the airliquid (AL) or liquid-liquid (LL) interfaces. In this case, metal ions and organic linkers present in different phases, coordinate at the interface to yield crystalline nanosheets. ${ }^{283}$ Multilayer, few or single layer nanofilms can form at the LL or AL interfaces This approach has been extensively developed specially for $2 \mathrm{D}$ CPs and MOFs, ${ }^{27}$ also known as coordination nanosheets (CONASHs), ${ }^{284,285}$ metalorganic layers (MOLs), ${ }^{286}$ metal-organic framework nanosheets $(\mathrm{MONs})^{287}$ or just low dimensional MOFs (LD MOFs). ${ }^{288}$ When films are pre-assembled at the LL interface, the procedure typically consists of carefully layering a solution of ligand in an organic solvent or a mixture of solvents onto a water solution of the metal salt, if the ligand solution is denser than water, then the metal solution is layered on top instead (Fig. 15). For instance, Sakamoto et al. prepared bis(dipyrrinato)zinc(II) complex micro and nanosheets containing zinc(II) porphyrin that could be layered quantitatively onto a flat substrate (Fig. 17). ${ }^{289}$ More sophisticated versions of this method include using a pure solvent as a diffusion barrier in between the two immiscible solvents that contain the
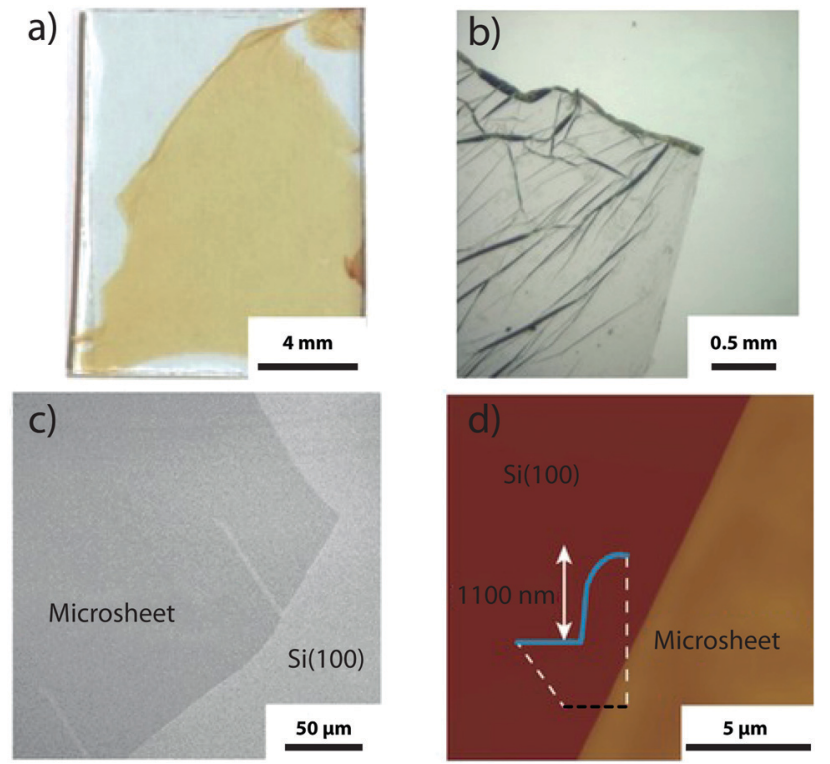

Fig. 17 (a) Representative photograph of a bis(dipyrrinato)zinc(II) microsheet transferred to a quartz substrate. (b) Optical microscopic image of the microsheet on a silicon (100) substrate. (c) SEM image of the microsheet on a silicon (100) substrate. (d) AFM image of the microsheet on a silicon (100) substrate with a cross-section profile (blue line) taken along the black line. Reproduced from ref. 289 with permission from Wiley, copyright 2017.

metal-organic building blocks ${ }^{139,140}$ or spraying the top solution instead of layering it with a syringe. ${ }^{290,291}$

The AL interfacial synthesis is similar to the LL version, a controlled amount of a diluted solution of a volatile organic solvent containing the ligand is carefully dispersed onto the surface of an aqueous solution of a metal salt. The organic solvent quickly evaporates leaving the ligand to react with the metal ions at the $\mathrm{AL}$ interface forming a floating layer of the $\mathrm{CP}$ or MOF in question. In any case, the final step involves the transfer of the films from the LL or AL interface to a solid substrate. This can be done by piercing the interfacial film with a substrate going either from the organic to the water solution or vice versa, by repeating the assembly-deposition process several times, the final film thickness can be controlled. Sometimes, in the LL version, the organic layer can be left to evaporate or syringed out, so the final transfer occurs through the AL interface. For these methods to produce single or few-layers films, the synthetic conditions (solvents, concentrations, reaction time, temperature, etc.) have to be carefully selected. In spite of this, it is not always possible to obtain them if interlayer interactions are strong. The thickness and lateral size of the nanosheets produced with these methodologies can usually vary between micro and nanometres. However, some works by Nishihara and collaborators have reported centimetre-sized and defect-free sheets by LL interfacial synthesis although additional crystallographic evidence would still be desirable in order to support these claims. ${ }^{285}$ Regarding conductive MOG systems, several of them have been prepared as nanosheets using LL interfacial synthesis, mainly by Nishihara and co-workers. Thus, the first 
system was Ni-BHT in $2013^{135}$ followed by $\mathrm{Pd}-\mathrm{BHT},{ }^{137} \mathrm{Cu}-$ BHT, ${ }^{131,145}$ Co-BHT, ${ }^{136}$ Ag-BHT, ${ }^{133}$ Ni-IT, ${ }^{139} \mathrm{Ni}-\mathrm{AT},{ }^{140} \mathrm{Ni}-\mathrm{HIB}, \mathrm{Cu}-$ HIB, Co-HIB, ${ }^{141}$ THTA-Co, ${ }^{147}$ Co-HTTP ${ }^{149}$ and Cu-CAT- $1 .{ }^{156}$

The obvious limitation of the interface synthesis is imposed by the size of the reaction vessel and the low control over the substrate coverage. There are no means to repair cracks or empty space that appear between individual sheets (Fig. 17). The use of organic solvents and the need to have a steady LL or AL interfaces to minimize defects also make these methods hardly compatible with high-temperature synthesis. In 2010, Makiura et al. introduced the Langmuir-Blodgett (LB) technique ${ }^{292}$ as an elegant method to overcome some of these limitations and gain control over the interfacial area coverage. ${ }^{293}$ The LB technique is an elegant method for the fabrication of well-defined layered structures with precision at a molecular level. A picture of a standard LB through is depicted in Fig. 18a. Once a floating CP or MOF has been formed at the AL interface, two barriers are used to reduce available interface area, thus bringing individual sheets together (Fig. 18b). Using a Wilhelmy plate, the changes in surface pressure $(\pi)$, defined as the difference in surface tension $(\sigma)$ measured between a clean subphase and a surfactant-covered subphase, can be measured. The plot of $\pi$ as a function of mean molecular area (MMA) at the interface, calculated from the known number of molecules dispersed in the area left available by the barriers, is known as a compression isotherm (Fig. 18c). The study of these isotherms renders very useful information about the behaviour of the floating layer. As shown in Fig. 18c, at the initial point of a $\pi$-MMA compression isotherm, $\pi$ is almost 0 as there is plenty of empty

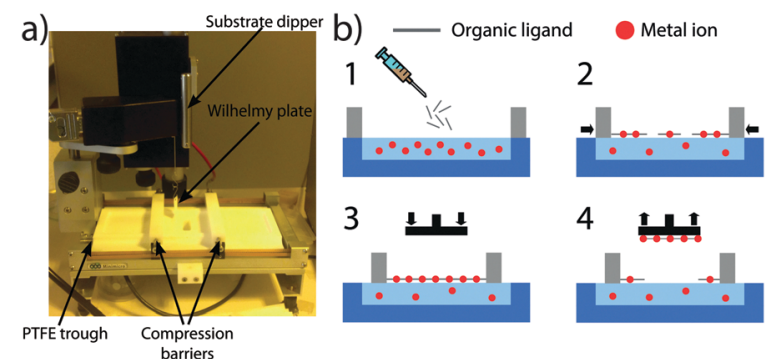

C)

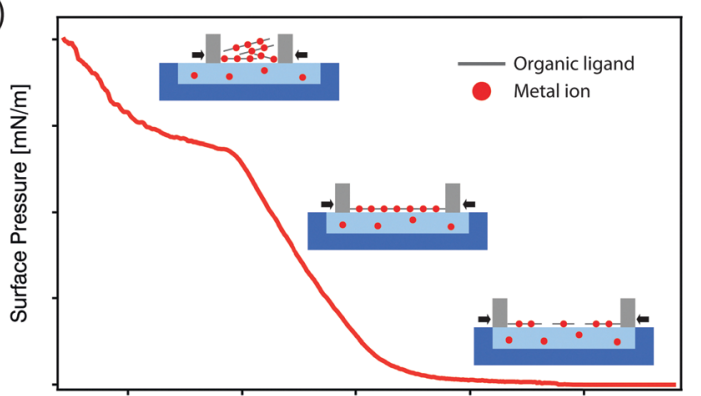

Mean Molecular Area $\left[\AA^{2} /\right.$ molecule $]$

Fig. 18 (a) Photograph of a Langmuir mini-trough. (b) Schematic illustration for MOF/CP nanosheet formation, compression and transfer to a substrate in a LB trough. (c) $\pi$-MMA compression isotherm with schemes illustrating the different stages of film growth with decreasing MMA, from the initially isolated nanosheets that are compressed to a continuous layer, to the final collapse state. space between the sheets of the dispersed layer. Then, as the sheets are brought closer together by barrier compression, $\pi$ increases as the available area and MMA decreases. Initially this raise is minimal as molecules still have plenty of space available (distance between adjacent floating sheets is large and their interactions are weak), there is little effect on the surface tension of the subphase. Then as MMA is further reduced and sheets start to adopt more compact packing (sheets begin exerting a repulsive effect on each other), the slope of the $\pi$-MMA isotherm becomes very steep. Finally, if the barriers keep advancing until the layer cannot be further compressed, it collapses forming either thicker solid aggregates or they submerge in the subphase by dissolution or micelle formation. This collapse produces an inflexion point in the isotherm, and if the molecule is coloured the collapse can also be observed by the naked eye.

The LB set-up also includes a dipping mechanism or dipper, which allows the transfer of the compressed floating film to the substrate, either with its surface parallel or perpendicular to the interface. This whole process can be repeated several times to achieve 2D CP or MOF ultrathin film of the desired thickness. There are two main transfer modes: vertical and horizontal. In the vertical transfer the substrate is firstly immersed through the floating film and then emerged or it can be already immersed before the floating film formation. In the first scenario, the floating film can be transferred to the substrate in the downwards and upwards motion. In the horizontal transfer mode or Langmuir-Schaefer deposition, ${ }^{294}$ the substrate's surface and the floating monolayer make contact parallel to each other, either by stamping the surface from above or by pulling the substrate from inside the subphase.

Early works by Makiura et al. reported the formation and transfer to a substrate of the layered $\mathrm{CP}\left[\mathrm{Cu}_{2}(\mathrm{py})_{4}{ }^{-}\right.$ (CoTCPP)], ${ }^{293,295,296}$ which curiously does not exist in the bulk form. Later, the same authors prepared a series of related porphyrin MOFs, including $\left[\mathrm{Cu}_{2}\left(\mathrm{H}_{2} \mathrm{O}\right)_{2}\left(\mathrm{H}_{2} \mathrm{TCPP}\right)\right]$ or NAFS-2, ${ }^{297}$ NAFS-21 ${ }^{298}$ with 5,10,15,20-tetra(4-pyridyl)-porphinato zinc(II), NAFS-13 or $\left[\mathrm{Cu}_{2}\left(\mathrm{H}_{2} \mathrm{O}\right)_{2}(\mathrm{PdTCPP})\right],{ }^{299}$ and NAFS-31 and $41,{ }^{300}$ which are equivalent to NAFS-2 but with trans-ditopic and expanded tetratopic porphyrins respectively. After NAFS-1, the LB technique has been used to fabricate ultrathin films of other MOFs. Feng and co-workers prepared single-layer sheets of the MOGs $\mathrm{Ni}_{3}(\mathrm{HTTP})_{2}{ }^{150}$ and THTA-Co ${ }^{147}$ but unfortunately the substrate coverage was not continuous. Nishihara and co-workers had done something analogous with Ni-BHT. ${ }^{301}$ Similar films of a different $2 \mathrm{D} \mathrm{Cu}{ }^{\text {II }}$ MOF were prepared by Ruoff and collaborators. ${ }^{302}$ Later Moradi et al. also prepared monolayers of a $2 \mathrm{D} \mathrm{Cu} \mathrm{Cu}^{\mathrm{II}} \mathrm{CP}$ with a complex 3D calix[4]arene amphiphile ligand. The carboxylate and alkyl chains in the ligand are located in opposing sides, thus guarantying a particular orientation on the water surface. ${ }^{303}$ In all these cases, the floating films were transferred to bare hydrophilic substrates (i.e. $\mathrm{Si} / \mathrm{SiO}_{2}, \mathrm{Au}$, quartz, etc.). Rubio-Giménez et al. later proved how the hydrophobicity of the substrates plays a key role in the transfer process by using NAFS-1 as a model system. ${ }^{304}$ Brewster angle microscopy (BAM) images of the floating film showed that it was continuous, but the final film once transferred to a bare 
hydrophilic substrate was rather discontinuous for the case of $\mathrm{Si} / \mathrm{SiO}_{2}$, whilst in the case of clean metallic substrates there was barely any transfer at all. By functionalizing these substrates with the appropriate alkyl self-assembled monolayers (SAMs), they turned highly hydrophobic and the transferred films were then continuous with full coverage for all surfaces. This hydrophobicity effect in LB transfer was latter replicated with Cu-CAT-1 films. ${ }^{156}$ The LB method is not exclusively limited to the bottom-up assembly of ultrathin nanosheets and can be also used to produce MOF thin films by sequential transfer of dispersions of NPs. ${ }^{305,306}$

4.1.2. Top-down deposition. Although extensively developed mainly for graphene, ${ }^{307-309}$ and inorganic $2 \mathrm{D}$ materials like transition metal dichalcogenides (TMDs), ${ }^{310,311}$ layered double hydroxides $(\mathrm{LDH}),{ }^{312}$ and many others, ${ }^{313,314}$ liquid phase exfoliation has also been used to prepare dispersions of layered CPs and MOFs that are then transferred to a substrate. Exfoliation in a liquid phase usually requires the assistance of ultrasonication to achieve delamination into a suspension that is easily processable. As a result, liquid exfoliation has been widely used to produce nanosheets of a variety of $2 \mathrm{D}$ CPs and MOFs, ${ }^{286,287,315}$ including a couple of MOGs ${ }^{153}$ and other conductive 2D frameworks. ${ }^{316}$ For instance, Amo-Ochoa et al. used sonication to delaminate a mixed valence copper $\mathrm{CP}\left[\mathrm{Cu}_{2} \mathrm{Br}(\mathrm{IN})_{2}\right]_{n}$ crystal into $5 \mathrm{~nm}$ sheets and deposited them as films on graphite surfaces (Fig. 19). ${ }^{109}$ Moreover, liquid exfoliation has also been used to prepare monolayer flakes of a 2D SCO material. Thus, Lei and Zheng exfoliated single layers of $\left[\mathrm{Fe}(4-\mathrm{PyP})\left(\mathrm{H}_{2} \mathrm{O}\right)\right]\left[\mathrm{Pt}(\mathrm{CN})_{4}\right] \cdot \mathrm{H}_{2} \mathrm{O} \cdot \mathrm{CH}_{3} \mathrm{OH}$ (4-PyP = diethyl 4-pyridylphosphonate), a $2 \mathrm{D} \mathrm{Fe}^{\mathrm{II}}-\mathrm{HCP}$ by ultrasonication in water. ${ }^{317}$ Ruiz-Molina and collaborators did the same with a different 2D CP. Despite thicknesses being rather polydisperse, they were still able to find few single layer flakes. ${ }^{318}$

However, indiscriminate sonication normally reduces the lateral size of the sheets from micro to few hundred nanometres and hinders their crystalline quality in comparison to those obtained by micromechanical exfoliation. As a result, a few examples of wet exfoliation that do not require ultrasonication have recently appeared. Sonication can be avoided by solvent-induced delamination ${ }^{320,321}$ or chemical exfoliation. ${ }^{322}$ From the chemical point of view, a particularly elegant example is the chemical exfoliation of $\left[\mathrm{Zn}_{2}(\mathrm{Pd}-\mathrm{TCPP})\right]$ recently reported by Zhou and co-workers. ${ }^{323}$ First, the $2 \mathrm{D}$ CP was intercalated with a bidentate $4,4^{\prime}$-dipyridyl disulphide ligand that coordinated to
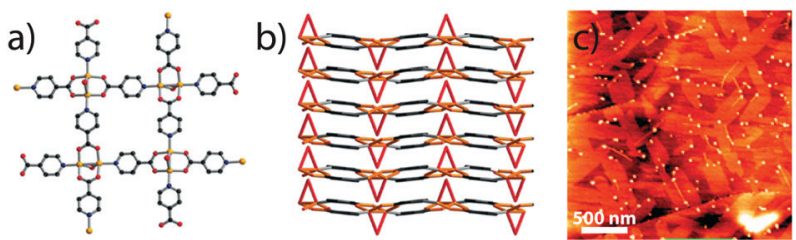

Fig. 19 (a) Detail view of the copper environment in a $\left[\mathrm{Cu}_{2} \mathrm{Br}(\mathrm{IN})_{2}\right]_{n}$ single layer, (b) superposition of layers along the a axis, (c) AFM topography image of $\left[\mathrm{Cu}_{2} \mathrm{Br}(\mathrm{IN})_{2}\right]_{n}$ after liquid phase exfoliation and deposition on HOPG. Reproduced from ref. 109 with permission from the Royal Society of Chemistry.
$\mathrm{Zn}^{\mathrm{II}}$ of adjacent layers, then the disulphide bond was broken by chemical reduction with trimethylphosphine, thus generating nanosheets in high yields. ${ }^{323}$

Liquid phase exfoliation does not require large single crystals as starting materials and is commonly considered as an efficient method to produce a big quantity of nanosheets in solution. Oppositely, solvent-free micromechanical exfoliation is the preferred method to produce highly crystalline nano and microsheets of a layered material. Physical exfoliation is done without chemical disturbance by applying simple mechanical force to a bulk crystal. The sheets deposited onto a substrate using this method are thus only formed by single crystalline domains. However, there are clear practical limitations to this method, from the difficulty in controlling the thickness and lateral size distribution of the exfoliated sheets (especially in the case of soft materials like CP and MOFs) to the low substrate coverages and exfoliation yields that can be achieved. Moreover, relatively large single crystals are needed for this method to work, particularly when using the scotch tape technique. This makes it especially challenging when dealing with CPs or MOFs, for which large crystallites are difficult to obtain. Still, there are a couple of interesting examples in the literature. Coronado and co-workers reported the first example of a mechanically exfoliated $\mathrm{CP}$ with $\left[\mathrm{Fe}\left(\mathrm{acac}_{2}\right.\right.$-trien $\left.)\right]\left[\mathrm{MnCr}\left(\mathrm{Br}_{2} \mathrm{An}\right)_{3}\right] \cdot\left(\mathrm{CH}_{3} \mathrm{CN}\right)_{2}$ which is formed by neutral honeycomb layers. Very recently, this team also achieved atomically thin microsheets of MUV-1, a family of $\mathrm{Fe}^{\mathrm{II}}$ benzimidazole 2D CPs, via mechanical exfoliation (Fig. 20). ${ }^{319}$

\subsection{In situ film growth in presence of the substrate}

In this strategy substrate and CP or MOF precursors share the same reactor space during material synthesis. In the simplest case, reaction conditions similar to those for bulk material synthesis are used, but in the presence of a substrate that can be chemically modified to assist and/or orient the film growth. When the reaction has finished, a film is formed on the substrate, most often along with a powder precipitated at the bottom

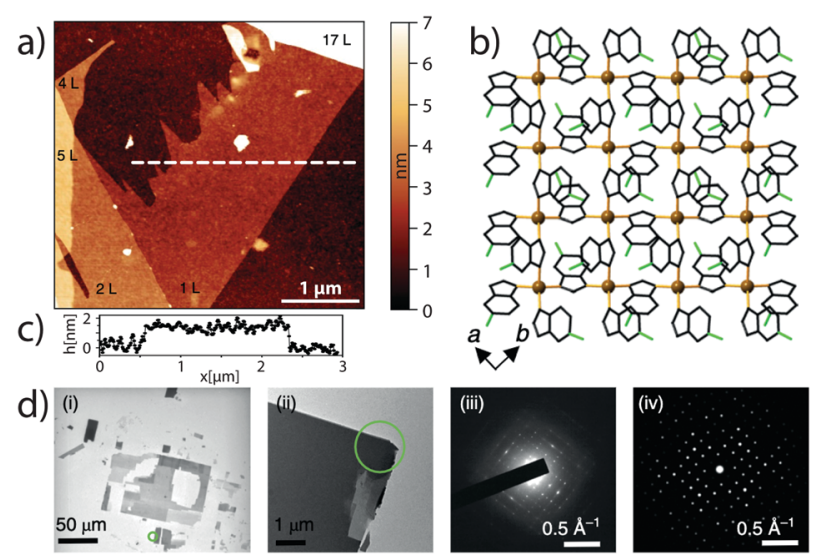

Fig. 20 Mechanical exfoliation of a layered CP into atomically thin sheets. (a) AFM image and height profile of a flake of MUV-1-Cl, (b) structure of a single layer of MUV-1-Cl viewed along the $c$ axis. (c) TEM images and (d) SAED patterns for MUV-1-Cl flakes. Reproduced from ref. 319 with permission from Springer Nature, copyright 2018. 

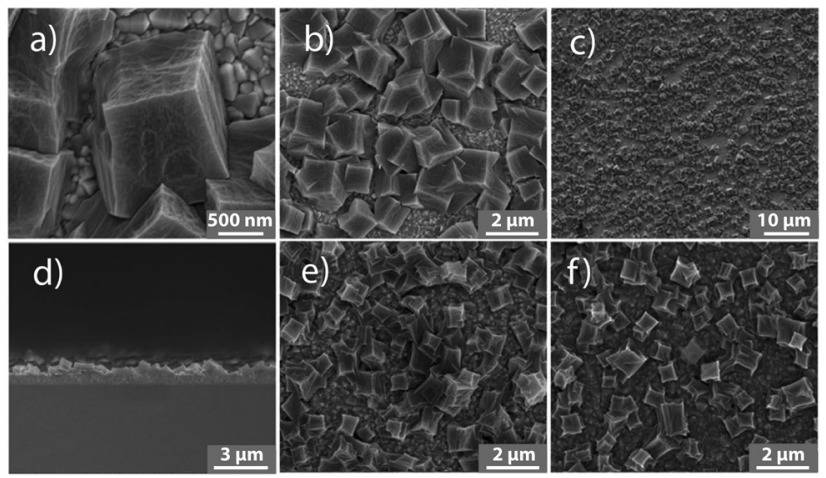

Fig. 21 SEM images of MOF-525 polycrystalline thick films prepared via solvothermally growth. Reproduced from ref. 326 with permission from the Royal Society of Chemistry.

of the reactor container. ${ }^{324,325}$ For example, Kung et al. used the solvothermal method to grow MOF-525 films on conducting glass substrates from its components $\mathrm{H}_{4}$ TCPP linkers and hexazirconium nodes). ${ }^{326}$ As visible in Fig. 21, cubic crystals ranging from $500 \mathrm{~nm}$ to $1000 \mathrm{~nm}$ embeded into each other all over the substrate.

Zacher et al. showed that the nature of the surface and especially its acid/base properties, influence whether a film can grow or not. The authors suggested that binding between the surface and the film is mediated by the organic linker, and therefore a MOF that contains acid linkers like HKUST-1 cannot grow on acidic surfaces such as silica. ${ }^{325}$ However, a MOF that contains both acidic and basic linkers such as $\left[\mathrm{Zn}_{2}(\mathrm{BDC}-\mathrm{Br})_{2}\right.$ (DABCO); (DABCO = 1,4-diazabicyclo[2.2.2] octane)] can growth on both silica an alumina. Arnold et al. reached similar conclusions by studying the anisotropic growth of $\mathrm{Mn}\left(\mathrm{HCO}_{2}\right)_{2}$ on alumina and graphite. ${ }^{324}$ As a result, the use of organic molecules has been proposed to improve heterogeneous nucleation and growth. Huang et al. treated a porous titania support with 3-aminopropyltriethoxysilane (APTES). This molecule can bind to the surface by the silane group and to ZIF-22 [Zn(ABLM) $]_{2}$; $(\mathrm{ABLM}=5$-azabenzimidazole $)$ through the terminal amino group. ${ }^{327}$ As a result, thick $(40 \mu \mathrm{m})$ and well-intergrown films of higher quality than the ones produced without the linker were obtained. The same phenomenon has been observed for ZIF-90 $\left[\mathrm{Zn}\left(\mathrm{C}_{4} \mathrm{H}_{3} \mathrm{~N}_{2} \mathrm{O}\right)_{2}\right]^{328}$

Usually, MOF synthesis takes place in solvothermal conditions. However, microwave-heating approaches have been developed for bulk materials synthesis and later extended to films. ${ }^{329}$ The advantage of this method over conventional heating is the increased nucleation rate. Indeed, a few hours is enough to archive full crystallization. This method was introduced by Yoo et al. using a substrate (porous alumina) coated with a conducting layer, such as graphite or gold, and immersed in a MOF-5 [ $\left.\mathrm{Zn}_{4} \mathrm{O}(\mathrm{BDC})\right]$ precursors solution. Upon microwave irradiation, the temperature of the conductive layer increased rapidly and introduced fast heterogeneous nucleation for MOF-5 crystals. ${ }^{330}$ Later, Bux et al. used this microwave-assisted deposition to prepare a $40 \mu \mathrm{m}$ thick ZIF-8 film on porous titania that exhibits molecular sieving properties. ${ }^{331}$
A variation of the above ideas consists on modifying reaction/ nucleation rates controlling the concentration/diffusion of one of the reagents involved. For instance, gel-layer synthesis can be used to better control nucleation over the surface. This method was originally applied to MOFs by Yaghi and co-workers to grow $\mathrm{Zn}$ (HBTC) $($ HBTC $=$ 5-carboxy-benzene-1, dicarboxylate dianion $){ }^{332}$ Later, Schoedel et al. employed it to grow HKUST-1 and Fe-MIL-88B- $\mathrm{NH}_{2}$ films on a $-\mathrm{COOH}$ or $-\mathrm{OH}$ terminal alkanethiolate SAM-functionalized gold substrates. They immersed the substrates in poly(ethylene oxide) gel loaded with metal ion precursor $\left(\mathrm{Cu}^{2+}\right.$ or $\left.\mathrm{Fe}^{3+}\right)$ and then carefully added the linker solution $\left[\mathrm{H}_{3} \mathrm{BTC}\right.$ or $\left.\mathrm{H}_{2}\left(\mathrm{NH}_{2}-\mathrm{BDC}\right)\right]$ on top of the gel layer. The morphology and thickness of the resulting MOF film could be controlled by the length of the poly(ethylene oxide) and the concentration of the metal ions in the gel. The thickness of the Fe-MIL-88B-NH ${ }_{2}$ film was estimated to be $40 \mathrm{~nm}^{333}$

An electrochemical method to control the reaction rate was originally developed by researchers at BASF. ${ }^{334}$ The principle was based on supplying the metal ion by anodic dissolution to a solution that contained the organic ligand and a conducting salt. Careful modification of the conditions used to electrochemically produce bulk MOF materials enabled the preparation of thin films. This process was demonstrated with HKUST-1, by applying an anodic voltage to the copper electrode in the presence of a solution containing BTC and methyltributylammnium methyl sulfate (MTBS) as conduction salt. Films of well-packed HKUST-1 crystals with thicknesses in the range 2 to $50 \mu \mathrm{m}$ were obtained by controlling the synthesis conditions. ${ }^{335}$

In situ growth can also be achieved by confining the reaction to the gas-solid interface, thus avoiding the use of solvents. Ameloot and collaborators used this approach to fabricate homogeneous pinhole-free $50 \mathrm{~nm}$ films of ZIF-8 with high-aspect ratio features in a highly controlled manner (Fig. 22a-e). An atomic layer deposited (ALD) nanometric ZnO film was reacted with the chemical vapour deposited (CVD) ligand to yield the final MOF film. ${ }^{336}$ Afterwards, this strategy has been optimized for ZIF-8 ${ }^{337-339}$ and successfully applied to many other MOFs. ${ }^{188,340-342}$ In the case of Nilsen and co-workers, they successfully produced UiO-66 and UiO-66 $\left(\mathrm{NH}_{2}\right)$ films over silicon substrates by exposing to acetic acid vapours $\mathrm{Zr}^{4+}$ BDC mixtures generated by the sequential atomic/molecular layer deposition (ALD/MLD) of $\mathrm{ZrCl}_{4}$ and $\mathrm{H}_{2} \mathrm{BDC}$ precursors over silicon substrates (Fig. 22f-h). ${ }^{343,344}$ Lastly, vapour-assisted conversion (VAC) is another thin film fabrication methodology at the edge of vapour and solution synthesis that was first developed for COFs by Medina and co-workers. ${ }^{345}$ In the MOF version, substrates coated with a drop of the transition metal/ligand precursor solutions are exposed to modulator/solvent vapour mixtures. The same team used VAC to successfully fabricate various 3D Zr-based MOFs (Fig. 22i-l) ${ }^{346}$ and 2D triphenylene MOGs (Co-CAT-1, Ni-CAT-1 and Cu-CAT-1). ${ }^{187}$ This VAC technique is highly versatile in terms of the dimensionality and nature of the MOFs that can be prepared and is not limited to sublimable building blocks, which is currently one of the main limitations of the CVD and ALD/MLD approaches.

\subsection{Sequential growth by layer-by-layer/liquid phase epitaxy}

A third strategy, the so-called LbL approach involves the sequential growth of the ultrathin film by consecutive exposure of the 
a)

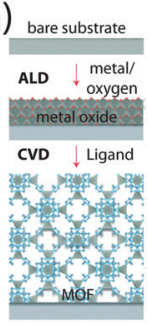

f)
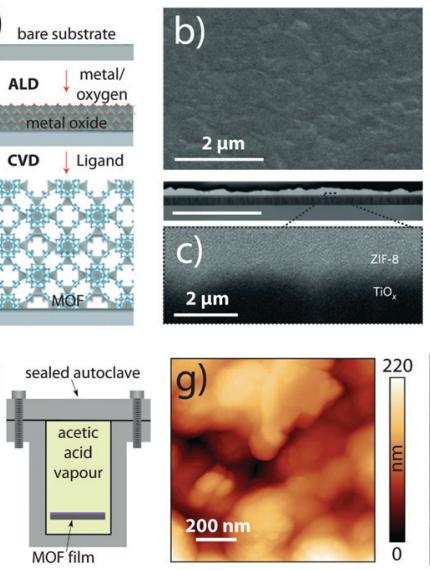

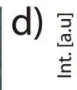

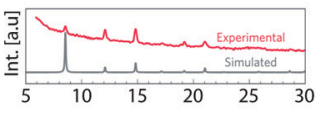

e)

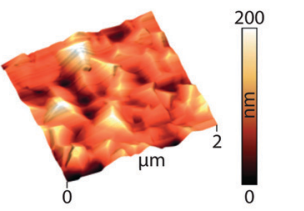

i)
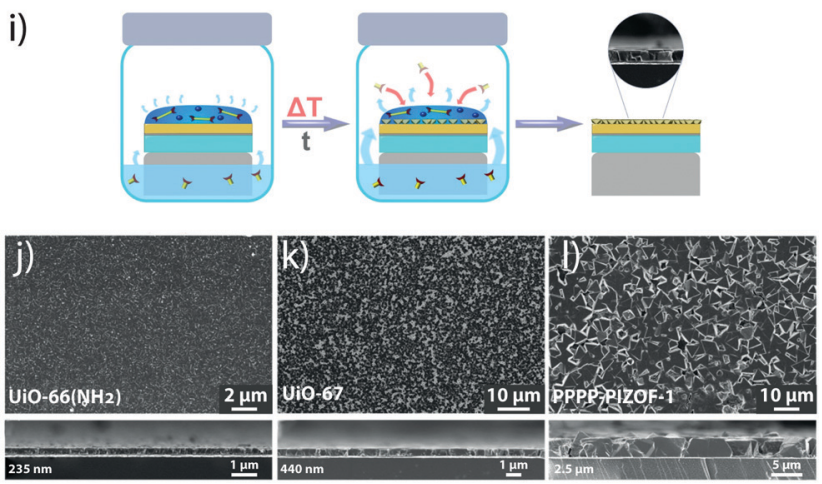

Fig. 22 (a) CVD of ZIF-8 thin films. The procedure consists of a metal oxide vapour deposition and a consecutive vapour-solid reaction. (b) SEM top view and (c) focused-ion beam TEM cross section images. Inset: Highresolution magnification of the interface between $\mathrm{ZIF}-8$ and the substrate. (d) XRD pattern of a ZIF-8 CVD film and simulated pattern for ZIF-8, (e) 3D rendered AFM topography. (f) Experimental set-up for heat postdeposition crystallization treatment of ALD/MLD Zr-1,4-BDC films in acetic acid vapour, (g) AFM image of the $\mathrm{Zr}-1,4-B D C$ film after treatment in acetic acid vapour, (h) cross-section SEM images of the same surface viewed at $45^{\circ}$ and $90^{\circ}$ angles. (i) Schematic representation of the VAC process for the fabrication of oriented MOF films. Top and cross-section SEM views of (j) UiO-66 $\left(\mathrm{NH}_{2}\right)$, (k) UiO-67 and (l) PPPP-PIZOF-1 films. (a-e) Adapted from ref. 336 with permission from Springer Nature, copyright 2016. (f-h) Adapted from ref. 343, licensed under CC BY 4.0, published by Springer Nature. (i-l) Reproduced from ref. 338 with permission from the American Chemical Society, copyright 2018.

substrate to the building blocks of the CP or MOF in question (Fig. 15). In this strategy, metal and ligand building blocks do not share the reaction space at the same time. The LbL procedure was pioneered by Mallouk and co-workers to fabricate thin films of various $\mathrm{CPs},{ }^{347-349}$ although it had been previously used for other materials. ${ }^{350}$ Years later, it was extensively employed by Fischer, Wöll and collaborators to fabricate MOF ultrathin films, also referred as surface mounted MOFs or SURMOFs. ${ }^{351-354}$ They also rebranded the technique as liquid phase epitaxy (LPE). ${ }^{355,356}$

In the simplest case, a LbL growth cycle consists of consecutive immersions of the substrate into solutions of the metal ion or the organic linker with intermediate washing steps using pure solvent. ${ }^{357,358}$ This gives each of the components the opportunity to saturate all deposition sites without the formation of new nuclei at the surface or in solution. Between each step, the sample is rinsed with solvent to remove uncoordinated precursors. Under ideal conditions, every cycle should imply the deposition of a single unit cell. This linear growth mode allows to adjust the final thickness of the film to the number of cycles. The previous functionalization of substrates with SAMs that have the appropriate head group to modify surface properties or coordinate to the metal ions and thus direct film growth for the following growth cycles is a common practice. ${ }^{359}$ The resulting films are in general highly crystalline, smooth, homogeneous and cover the whole area of the substrates that was functionalized with the appropriate head groups, ${ }^{351,353-355,360-362}$ and is thus compatible with the deposition of patterned features. ${ }^{360}$ The head group and the packing of the SAM also controls orientation of the resulting MOF or $\mathrm{CP}^{363}$ However, most studies have only focused on the out-of-plane orientation. Interestingly, heteroepitaxial methods that use oriented hydroxide precursors matching the MOF's lattice parameters have also been developed to control both in-plane and out-of-plane orientations. ${ }^{364,365}$

Concerning LbL experimental procedures, a simple manual dipping method was first reported by Mallouk et al. for the sequential deposition of $\left[\mathrm{Ni}(\mathrm{bpy}) \mathrm{Pt}(\mathrm{CN})_{4}\right]$ (bpy $=2,2^{\prime}$-bipyridine). ${ }^{349}$ Wöll, Ocal and co-workers used the dipping process to selectively graft HKUST-1 over $\mathrm{COOH}$ regions of a gold mercaptohexadecanoic acid (MHDA)/hexadecane thiol (HDC) patterned substrate. ${ }^{366,367}$ The substrate was sequentially immersed in $\mathrm{Cu}(\mathrm{OAc})_{2}$ and $\mathrm{H}_{3} \mathrm{BTC}$ ethanolic solutions, between each immersion the sample was rinsed with ethanol and dried with $\mathrm{N}_{2}$ stream. This same dipping process was later automatized by $\mathrm{Gu}$ et al. using an automatic robot dipper. Moreover, they found that the MOF films that were prepared under ultrasonication had cleaner and more homogenous surfaces and reduced their root mean square roughness from 38 to $10 \mathrm{~nm}$ (Fig. 23). ${ }^{368}$

A continuous flow method was used by Wöll and co-workers to synthesize $\mathrm{Cu}$ and $\mathrm{Zn}-\mathrm{MOF}-2$ [M(BDC); $\mathrm{M}=\mathrm{Cu}$ or $\mathrm{Zn}]$

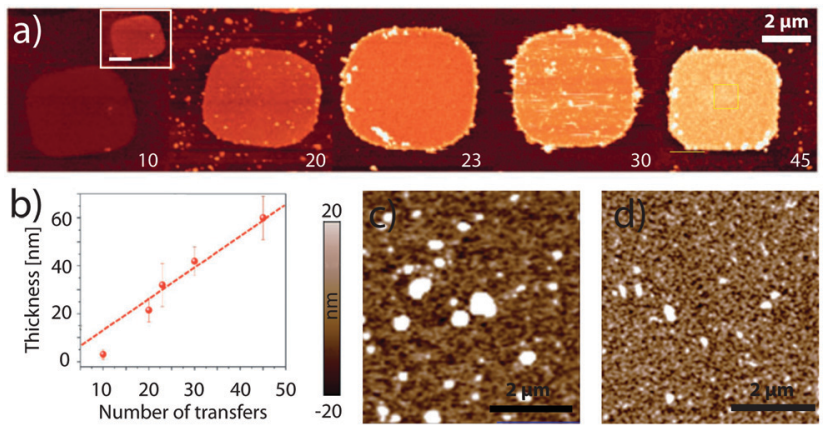

Fig. 23 (a) Series of topographic AFM images for different HKUST-1 samples deposited on a MHDA/HDC patterned substrate corresponding to $10,20,23,30$ and 45 immersion cycles. The colour scale (total height range) is $110 \mathrm{~nm}$ for all the images. Because of the low topography of the 10 cycles sample, the inset shows the same image with the scale magnified by a factor of two, inset scale bar is $2 \mu \mathrm{m}$. (b) Film thickness as a function of the number of immersion cycles. AFM images of HKUST-1 SURMOFs prepared without (c) and with ultrasonication (d). The surface roughnesses are $35 \mathrm{~nm}$ (c) and $6 \mathrm{~nm}$ (d). (a and b) Adapted from ref. 367 with permission from the PCCP Owner Societies. (c and d) Adapted from ref. 368 with permission from Elsevier, copyright 2015. 
ultrathin films. ${ }^{369}$ In this report, four pumps were connected to a sealed chamber. Two pumps were used for controlling the reaction time with the precursor solutions of $\mathrm{Cu}(\mathrm{OAc})_{2}(\mathrm{M}=\mathrm{Cu}$ or $\mathrm{Zn}$ ) and $\mathrm{H}_{2} \mathrm{BDC}$ to 30 and 60 minutes respectively. Another one, controlled the rinsing time, and the fourth one was responsible for pumping out the solution from the reactor. As shown in Fig. 24a, this method has also been used to epitaxially grow other 2D SURMOFs such as Zn-TCPP, in which consecutive layers are connected by van der Waals forces. ${ }^{370}$ In addition, quartz crystal microbalance (QCM) and surface plasmon resonance (SPR) have been widely reported as complementary methods to monitor the amount of material deposited during the process. For example, Shekhah recorded the sequential injection of $\mathrm{Cu}(\mathrm{OAc})_{2}$ and $\mathrm{H}_{3} \mathrm{BTC}$ solutions along with ethanol rinsing on a functionalized QCM electrode. ${ }^{371}$ Moreover, these techniques can be used to obtain information about film porosity after the growth is finished. ${ }^{306,335,372-379}$

The spray method is related to the dipping method. ${ }^{382}$ In this case, a spray system is adapted to dispense the reagents (Fig. 24b). This approach has been used to grow MOF ultrathin film with fast speed whilst keeping a high degree of crystallinity and orientation. In this method, there are three nozzles for spraying the solutions including metal salts, organic ligands, and rising solvent, respectively. As a proof of concept, HKUST-1 was again selected to demonstrate the efficiency of this method by Wöll's group. ${ }^{382}$ Carboxylic acid terminated SAM-modified substrates were alternately exposed to aerosol mixtures containing
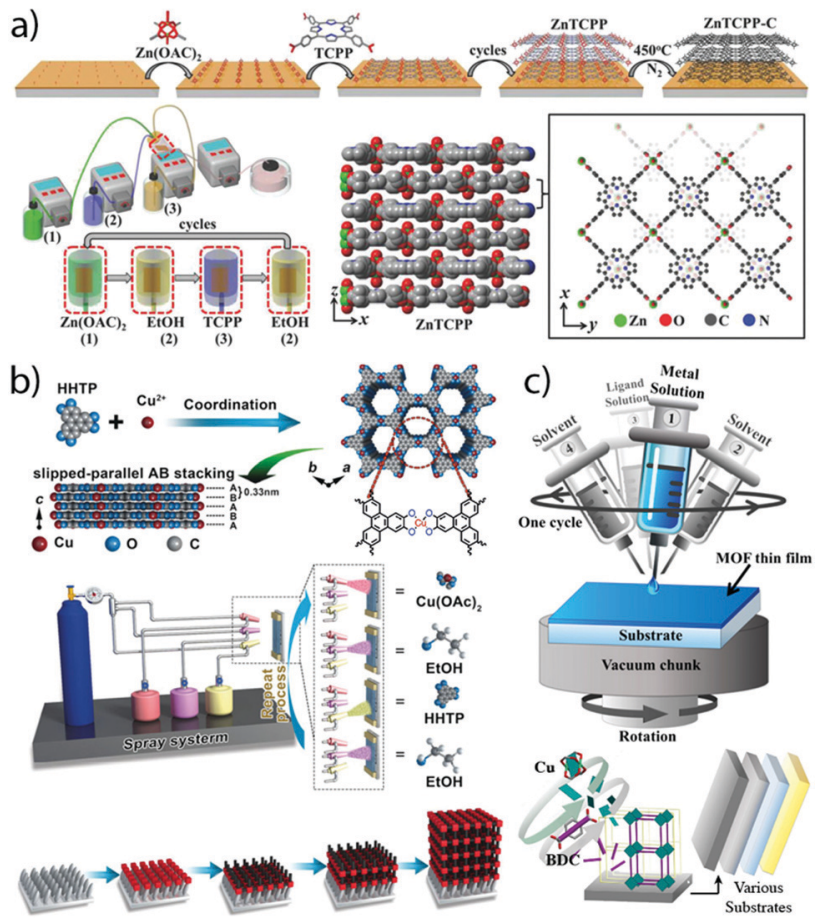

Fig. 24 Examples of LbL fabrication methods by (a) continuous flow, (b) controlled spraying and (c) sequential spin-coating of Zn-TCPP, Cu-CAT-1 and $\mathrm{Cu}_{2}(\mathrm{bdc})_{2} \cdot \mathrm{xH}_{2} \mathrm{O}$ thin films respectively. (a) Reproduced from ref. 370 with permission from Wiley, copyright 2018. (b) Reproduced from ref. 380 with permission from Wiley, copyright 2017. (c) Reproduced from ref. 381 with permission from the American Chemical Society, copyright 2016.
$\mathrm{Cu}(\mathrm{OAc})_{2}$ or $\mathrm{H}_{3} \mathrm{BTC}$ produced by spray nozzles. Like the other methods, the removal of residual reactants (metal ions, organic linkers) was achieved by exposing the substrate to an aerosol produced from the pure solvent. As depicted in Fig. 24b, controlled spraying LbL can also be used to produce films of $2 \mathrm{D}$ systems such as Cu-CAT-1. ${ }^{380}$ Also, the spin-coating method is an effective way to prepare thin and homogeneous films out of precursor solutions on flat substrates. In 2009, Vozar et al. reported an automated spin-assisted LbL assembly. ${ }^{383}$ In this system, a substrate is rotated rapidly, and precursors and cleaning solutions are deposited onto the substrate, typically using a nozzle impinging upon the center of the substrate. The rapid spinning of the sample produces a thin layer over the full substrate area. This setup was originally used to fabricate polymer-clay nanocomposites and was recently adapted by Chernikova et al. to grow $\mathrm{Cu}_{2}(\mathrm{BDC})_{2} \cdot x \mathrm{H}_{2} \mathrm{O}$ (Fig. 24c), ZIF-8 and HKUST-1 ultrathin films. ${ }^{381}$

The LbL method has also been extensively applied to SCO films of $\mathrm{Fe}^{\mathrm{II}}$-HCPs. After the first HCP films of [Ni(bypy)Pt $\left.(\mathrm{CN})_{4}\right]$ were grown by Mallouk and co-workers, ${ }^{349}$ a series of $3 \mathrm{D} \mathrm{Fe} \mathrm{Fe}^{\mathrm{II}}$ HCPs films were grown by Bousseksou, Real and collaborators. These include $\left[\mathrm{Fe}(\mathrm{pz}) \mathrm{Pt}(\mathrm{CN})_{4}\right],^{384,385}\left[\mathrm{Fe}(\operatorname{azpy}) \mathrm{M}(\mathrm{CN})_{4}\right](\mathrm{M}=\mathrm{Ni}$, $\mathrm{Pd}$ or $\mathrm{Pt})^{386}$ and $\left[\mathrm{Fe}(\mathrm{bpac}) \mathrm{Pt}(\mathrm{CN})_{4}\right]^{226,387}$ Afterwards, Kitagawa and co-workers structurally validated the growth of $\left[\mathrm{Fe}(\mathrm{pz}) \mathrm{M}(\mathrm{CN})_{4}\right]$, proving via synchrotron XRD that the films were crystalline and preferentially oriented with the metal-cyanide layers parallel to the substrate. ${ }^{388,389}$ They also reported the oriented growth of a new accordion-like 3D $\mathrm{Fe}^{\mathrm{II}}$-HCP: $\left[\mathrm{Fe}\left(\mathrm{H}_{2} \mathrm{O}\right)_{2}(\mathrm{bpy})\right]\left[\mathrm{Pt}(\mathrm{CN})_{4}\right] \cdot \mathrm{H}_{2} \mathrm{O} \cdot{ }^{390}$ Unfortunately, due to the fast exchange dynamics for $\mathrm{Fe}^{2+}$, the $\mathrm{LbL}$ procedure for all these $3 \mathrm{D} \mathrm{Fe} \mathrm{Fe}^{\mathrm{II}}$-HCPs, requires very low temperatures $\left(-60{ }^{\circ} \mathrm{C}\right)$ in order to achieve a regular epitaxial growth and avoid $\mathrm{Fe}^{2+}$ desorption in the washing steps. Kitagawa and co-workers later extended this LbL method to ultrathin films $\left(16 \mathrm{~nm}\right.$ thick) of $2 \mathrm{D} \mathrm{Fe}^{\mathrm{II}}-\mathrm{HCPs}:\left[\mathrm{Fe}(\mathrm{py})_{2} \mathrm{Pt}(\mathrm{CN})_{4}\right]^{244,391}$ and $\left[\mathrm{Fe}(\mathrm{py})_{2} \mathrm{Ni}(\mathrm{CN})_{4}\right],{ }^{392}$ which could be grown at room temperature unlike their 3D counterparts. Moreover, $\left[\mathrm{Fe}(\mathrm{py})_{2} \mathrm{Pt}(\mathrm{CN})_{4}\right]$ films below a certain thickness limit $(<22 \mathrm{~nm})$ displayed a gateopening behaviour that allowed solvent uptake in-between the layers of this non-intrinsically porous framework. ${ }^{244}$ Kitagawa's group procedure for $\left[\mathrm{Fe}(\mathrm{py})_{2} \mathrm{Pt}(\mathrm{CN})_{4}\right]$ films was latter improved by Rubio-Giménez et al. by adapting the LbL set-up into a $\mathrm{N}_{2}$ filled glovebox, thus avoiding $\mathrm{Fe}^{2+}$ oxidation and improving the quality of the films in the ultrathin film range $(\sim 1-22 \mathrm{~nm}) .^{393}$ The same research team also extended this procedure to two other $2 \mathrm{D} \mathrm{Fe}{ }^{\mathrm{II}}$-HCPs $\left[\mathrm{Fe}(\text { pym })_{2} \mathrm{Pt}(\mathrm{CN})_{4}\right]$ and $\left[\mathrm{Fe}(\text { isoq })_{2} \mathrm{Pt}(\mathrm{CN})_{4}\right]$ in a study of the influence of the axial ligand onto the gate-opening solvent uptake. $^{246}$

The ALD/MLD technique can also be used in a LbL fashion to build CPs and MOFs in a more aligned way to current CMOS technologies. ${ }^{25,394}$ Karppinen and co-workers have been working extensively on this procedure, which involves consecutive selfterminating reactions. Thus, in it the framework is sequentially built via independent gas pulses of the organic ligand and a reactive metal precursor. This procedure has been moderately successful, as most of the prepared films are either amorphous, ${ }^{395-398}$ require a separate recrystallization under a solvent atmosphere ${ }^{399-401}$ or their diffractograms do not clearly match a known structure. ${ }^{402-404}$ 


\subsection{Nanostructuration effects on the spin crossover transition}

The integration of $\mathrm{Fe}^{\mathrm{II}}$ SCO materials in functional nanodevices has attracted substantial attention in recent years. ${ }^{29,201-204,405}$ However, there is still a long way to go and additional efforts need to be made in the study of the relationship between physical properties and the spin state. Processing SCO materials at the nanometric scale has proven to have very significant effects on all aspects of the SCO phenomena. ${ }^{29,406-410}$ These include: (i) the stabilization of one of the spin states, which affects the transition temperature; (ii) the cooperativity between the SCO centers, which affects the abruptness of the transition and the width of its hysteresis; and (iii) the presence of defects, which affects the completeness of the transition. These properties can be altered by changes in the film thickness, sizes and shapes of crystallites, film orientation, substrate coverage and interactions with other SCO particles or non-SCO substrates or matrixes. Understanding the relationship between the characteristics of the film and the SCO properties is a key requirement to fabricate viable devices.

Regarding SCO CPs and MOFs, again most studies have been done with the $1 \mathrm{D} \mathrm{CP}\left[\mathrm{Fe}(\mathrm{Htrz})_{2}(\mathrm{trz})\right]^{+}$and $\mathrm{Fe}^{\mathrm{II}}-\mathrm{HCPs}$, for which there is an extensive amount of reports about nanostructuration effects on NPs already covered by various reviews. ${ }^{29,406-410}$ Nonetheless, the study of NPs adds other variables to the problem that are difficult to control such as size polydispersity, chemical composition changes or surface defects. The main consequence of nanostructuration is the size reduction effects, which can be divided into surface and confinements effects. In general, the first ones dominate for particles larger than $5-10 \mathrm{~nm}$, whilst confinements effects only acquire importance in smaller particles. ${ }^{29}$

Largely, experimental observations in NPs relate the decrease in particle sizes (increase of surface-to-volume ratio) with a decrease in cooperativity (increasingly gradual SCO transitions and narrower hysteresis), lower transition temperatures (stabilization of the HS state) and less complete transitions (increase of the residual HS fraction). This has been consistently observed for both $2 \mathrm{D}$ and $3 \mathrm{D}$ $\mathrm{Fe}^{\mathrm{II}}$-HCPs. For instance, in NPs of $\left[\mathrm{Fe}(\mathrm{pz}) \mathrm{Pt}(\mathrm{CN})_{4}\right] \cdot n \mathrm{H}_{2} \mathrm{O}$ studied both by the groups of Real and Mallah. The former prepared surfactantfree particles of $230 \times 230 \times 55 \mathrm{~nm}$ and $61 \times 61 \times 21 \mathrm{~nm},{ }^{411}$ whilst the latter studied much smaller particles (7 and 14 nm, Fig. 25a). ${ }^{412}$
Regardless, both observed a decrease in cooperativity, lower transition temperatures and an increase in the residual HS fraction, which went along with the decrease in particle size. Similar results were obtained by Real, Gaspar and collaborators with a series $2 \mathrm{D} \mathrm{Fe}^{\mathrm{II}}$ HCP: $\left[\mathrm{Fe}(3-\mathrm{Fpy})_{2} \mathrm{M}(\mathrm{CN})_{4}\right]$ (3-Fpy = 3-fluoropyridine; $\mathrm{M}=\mathrm{Ni}, \mathrm{Pd}$, $\mathrm{Pt})$. The SCO properties of surfactant-free nanocrystals of $400 \times$ $400 \times 30 \mathrm{~nm}$, were compared with NPs in the range $200-70 \mathrm{~nm}$ prepared using the coating polymer poly(vinylpyrrolidone) (PVP). ${ }^{413}$ Rubio-Giménez et al. also observed the same effect with nanocrystals of $\left[\mathrm{Fe}(\mathrm{py})_{2} \mathrm{Pt}(\mathrm{CN})_{4}\right]$ (Fig. 25b). ${ }^{393}$ On the other hand, confinement effects manifested in ultrasmall particles of $\left[\mathrm{Fe}(\mathrm{pz}) \mathrm{Ni}(\mathrm{CN})_{4}\right]$ prepared by Bousseksou, Salmon and co-workers. They observed a higher transition temperature in 2-4 nm NPs and even the reappearance of a hysteresis loop. ${ }^{414,415}$ However, it must be noted that the chemical composition of the NPs and the surfactant were not constant in these cases. Per contra, although their SCO properties follow the same trend with downsizing, NPs of $\left[\mathrm{Fe}(\mathrm{Htrz})_{2}(\mathrm{trz})\right]^{+}$compounds seem to be much less sensitive to size reductions effects. ${ }^{254,416}$ For example a hysteresis of $24 \mathrm{~K}$ was detected for $4 \mathrm{~nm}$ NPs (Fig. 25c). ${ }^{417}$ This can be rationalized in terms of the relative number of $\mathrm{Fe}^{\mathrm{II}}$ centres potentially exposed to surface effects. This is logically higher for $2 \mathrm{D}$ and $3 \mathrm{D} \mathrm{Fe}{ }^{\mathrm{II}}$ HCPs than for a 1D chain CP. Strangely, a recent report by Galán-Mascarós and co-workers showed that particle size reduction and defect generation in $\left[\mathrm{Fe}(\mathrm{trz})(\mathrm{Htrz})_{2}\right]\left[\mathrm{BF}_{4}\right]$ nanocrystals via ball milling, caused a wider hysteresis loop without affecting the completeness of the SCO transition. ${ }^{418}$ The physical environment around the particles has also got a significant influence on the SCO transition. Several reports have examined these matrix effects, ${ }^{414,419}$ the magnitude of which depends on the chemical and physical properties of the matrix, which helps or hinders the propagation interactions of the SCO transition. A matrix can be viscous liquid media, an organic polymer or a shell attached to the NP itself. Moreover, the matrix can govern interparticle interactions promoting cooperativity by transmitting the elastic vibrations of the spin state switching. ${ }^{420}$

In addition to NPs, there has been extensive work in the literature on ultrathin films of discrete $\mathrm{Fe}^{\mathrm{II}}$ complexes prepared by vacuum sublimation. Thus, few-layer to sub-monolayer films have been prepared onto different substrates and their SCO
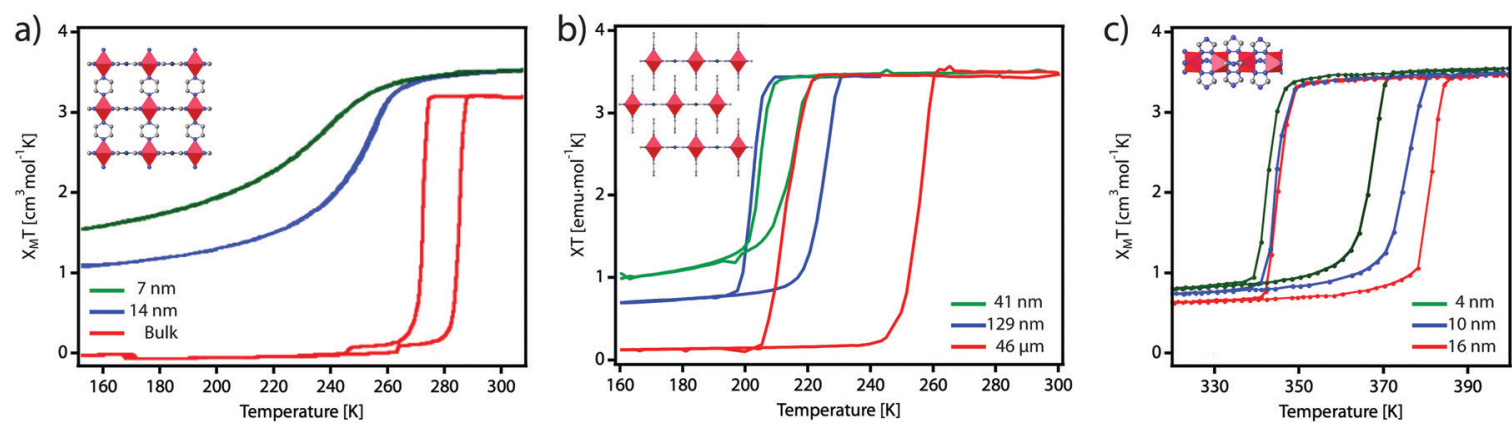

Fig. 25 Evolution of the temperature dependence of the molar magnetic susceptibility for SCO NPs of different sizes of $\left[\mathrm{Fe}(\mathrm{pz}) \mathrm{Pt}(\mathrm{CN})_{4}\right]$, a 3D Fe" $-\mathrm{HCP}$ (a); $\left[\mathrm{Fe}(\mathrm{py})_{2} \mathrm{Pt}(\mathrm{CN})_{4}\right]$, a 2D Fe" $-\mathrm{HCP}(\mathrm{b})$; and $\left[\mathrm{Fe}(\mathrm{Htrz})_{2}(\mathrm{trz})\right]\left(\mathrm{BF}_{4}\right)$, a $1 \mathrm{D} \mathrm{CP}(\mathrm{c})$. Insets show the crystal structures of the different materials. (a) Adapted from ref. 412 with permission from the American Chemical Society, copyright 2008. (b) Adapted from ref. 393, licensed under CC BY 3.0, published by the Royal Society of Chemistry. (c) Adapted from ref. 417 with permission from the Royal Society of Chemistry. 
properties examined using STM and XAS. Thermal evaporation of these complexes allows for a precise control over film thickness. However, it requires complex equipment and is limited to a relatively small collection of sublimable compounds. Furthermore, the resulting films are typically amorphous, unless they are recrystallized afterwards, ${ }^{421,422}$ which in combination with interactions with the substrate can strongly affect their SCO properties. These studies are out of the scope of this work and been recently reviewed by Ruben and Kumar. ${ }^{423}$

In contrast with NPs and discrete complexes, the nanostructuration effects in crystalline thin films of SCO MOFs and CPs have been largely overlooked. As discussed in the previous sections, in the category of SCO MOFs and CPs, mainly $\mathrm{Fe}^{\mathrm{II}}$ HCPs have been grown onto solid substrates using the LbL method. As with thermal evaporation, LbL also allows for a precise control of film thickness by defining the number of growth cycles. Furthermore, LbL yields highly crystalline films with preferential orientation with respect to the substrate. The study of the magnetic properties of this $\mathrm{Fe}^{\mathrm{II}}$-HCPs thin films at the nanometric limit can be considered a challenging task, as conventional techniques such as SQUID magnetometry or Raman spectroscopy are not sensitive enough. Both of them require relatively thick films to get a good signal-to-noise ratio. Thus, films of a minimum thickness close to $100 \mathrm{~nm}$ are often required to observe the SCO transition, far from the threshold for which nanostructuration effects are expected to appear. This is the case for the series of 3D Fe ${ }^{\mathrm{II}}$-HCP thin films reported by Real, Bousseksou and collaborators which were studied in that thickness range. Consequently, authors barely noticed any difference in the SCO properties between bulk references and thin films of $\left[\mathrm{Fe}(\mathrm{pz}) \mathrm{Pt}(\mathrm{CN})_{4}\right],{ }^{384}\left[\mathrm{Fe}(\right.$ azpy $\left.) \mathrm{M}(\mathrm{CN})_{4}\right](\mathrm{M}=\mathrm{Ni}, \mathrm{Pd}$ or $\mathrm{Pt}),{ }^{386}\left[\mathrm{Fe}(\mathrm{bpac}) \mathrm{Pt}(\mathrm{CN})_{4}\right],{ }^{226,387}$ except for a slight change in the transition temperatures. The $\left[\mathrm{Fe}(\mathrm{bpac}) \mathrm{Pt}(\mathrm{CN})_{4}\right]$ publication features the thinnest film measured via Raman spectroscopy (16 nm, 10 growth cycles), which also does not show any significant difference with the SCO behaviour of the corresponding bulk reference sample. ${ }^{387}$

In the only study to date of a $2 \mathrm{D} \mathrm{Fe} \mathrm{Fe}^{\mathrm{II}}-\mathrm{HCP}$ in the ultrathin film range $(<20 \mathrm{~nm})$, Martí-Gastaldo and collaborators studied the dependence of the SCO properties of $\left[\mathrm{Fe}(\mathrm{py})_{2} \mathrm{Pt}(\mathrm{CN})_{4}\right]$ with the film thickness (Fig. 26). Variable temperature XAS was used to characterize the spin state in the $\sim 1-22 \mathrm{~nm}$ thickness range (1-30 LbL growth cycles). Thus, the authors analysed the XAS spectra together with the AFM characterization of the films and observed a dependence of the film morphologies with their SCO properties. From below 15 growth cycles $(<12 \mathrm{~nm})$, there is a dramatic decrease in the cooperativity and the completeness of the transition, which coincides with a microstructural change in the films. The LbL growth process generates partially segregated crystallites surrounded by a matrix of $\mathrm{Fe}^{\mathrm{II}}$ centres blocked in the HS state. Around the referred thickness threshold, these crystallites coalesce into a homogeneous film. Curiously, as the thickness gradually increases (one unit cell per growth cycle) the crystallite lateral size does not significantly change with the successive growth cycles. Thus, the thickness influence on the SCO properties cannot be attributed to a size
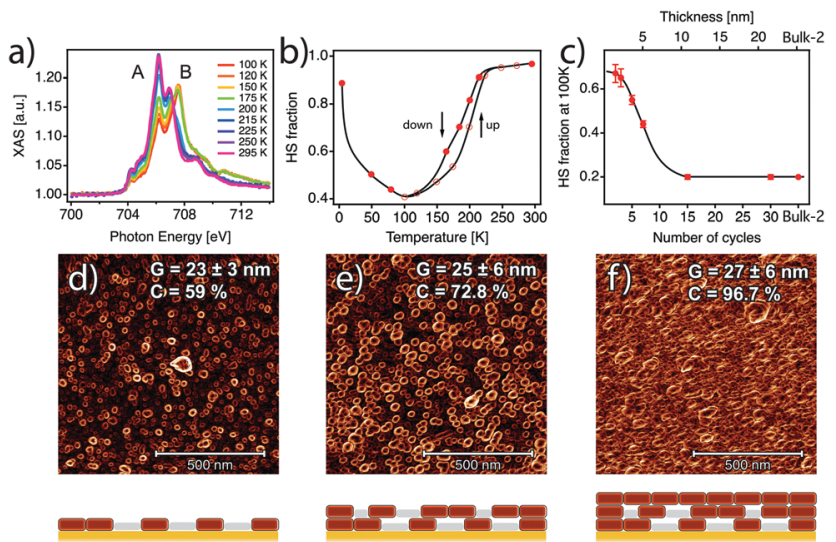

Fig. 26 SCO behaviour of $\left[\mathrm{Fe}(\mathrm{py})_{2} \mathrm{Pt}(\mathrm{CN})_{4}\right]$ ultrathin films analysed with XAS data and correlation with film microstructure. (a) XAS spectra as a function of temperature for a 7-cycles $\left[\mathrm{Fe}(\mathrm{py})_{2}\left\{\mathrm{Pt}(\mathrm{CN})_{4}\right\}\right]$ thin film. (b) $\mathrm{HS}$ fraction as function of the temperature for the 7-cycles film. (c) HS fraction at $100 \mathrm{~K}$ as a function of the number of cycles ( 1 cycle $\approx 0.72 \mathrm{~nm}$ ). $1 \times 1 \mu \mathrm{m}^{2}$ AFM topography images of 2 (d), 5 (e) and 10 (f) cycles after being processed with a Prewitt operator to highlight particle edges. The changes in the microstructure of the films with the number of cycles were monitored by analysing mean lateral grain size (G) and film coalescence (C) on $1 \times 1 \mu \mathrm{m}^{2}$ AFM images. Schematic illustrations of the microstructure of each film are shown below each AFM image, showing the evolution from a film composed by isolated nanocrystals to a fully coalesced one. Adapted from ref. 393, licensed under CC BY 3.0, published by the Royal Society of Chemistry.

effect, but to interparticle interactions which enhance cooperativity and reduce the residual HS fraction.

We believe that these works represent an initial step on the necessary understanding of the SCO phenomenon at the ultrathin film limit, concomitant to the use of these materials on functional nanodevices. However, further joint work by the SCO and the MOF/CP research communities should follow in order to achieve this goal.

\section{Integration of conductive MOFs and SCO CPs in functional devices}

As previously mentioned, the path to integrate these molecular materials in functional devices is already being paved. ${ }^{24,25,57,58}$ Two of the devices that have centered applications of conductive frameworks are FETs and chemical sensors, whereas SCO has been almost exclusively used to transduce magnetic bistability into rectified mechanical, optical or electrical responses.

\subsection{Field-effect transistors}

FETs are three-terminal circuit elements that use electric fields to modulate the electrical behaviour of the device. A FET consists of a semiconductor or active channel, a dielectric and three conducting electrodes (gate, source and drain). Source and drain electrodes are connected to the semiconductor through ohmic contacts. The conductivity of the channel is a function of the potential applied across the gate and source terminals. In practical digital circuits they operate as logic 
a)
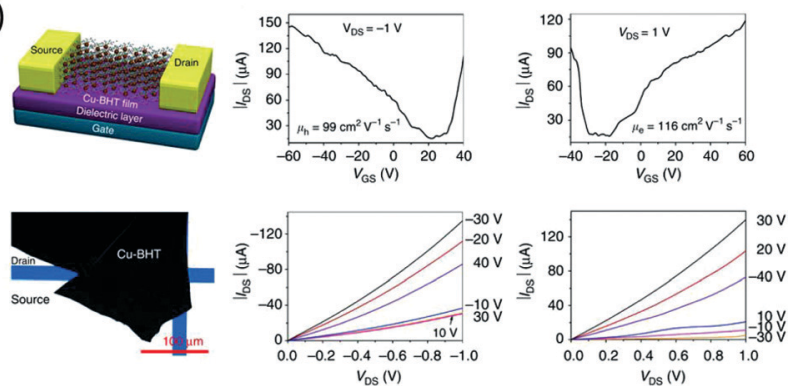

b)
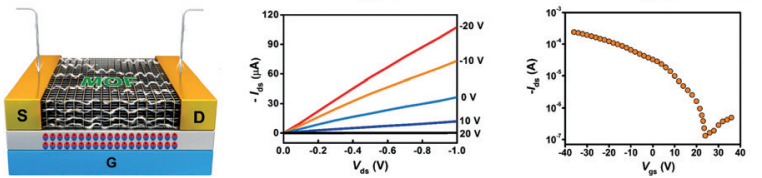

C)
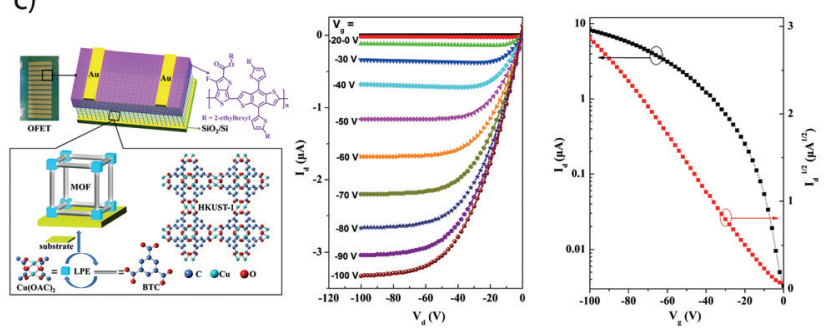

Fig. 27 Selected examples of MOF-FETs. Schematic diagrams of device architectures for MOF-FET devices composed of $\mathrm{Cu}-\mathrm{BHT}$ (a) or $\mathrm{Ni}_{3}(\mathrm{HITP})_{2}$ (b) as active channels and HKUST-1 as bottom dielectric (c) along with output and transfer characteristics. (a) Reproduced from ref. 131, licensed under CC BY 4.0, published by Springer Nature. (b) Reproduced from ref. 425 with permission from the American Chemical Society, copyright 2016. (c) Reproduced from ref. 424 with permission from the American Chemical Society, copyright 2017.

gates. In electronics research, FETs are also a useful tool to characterize the electronic properties of semiconductors, as key parameters such as charge carrier mobilities and threshold voltages can be extracted from them. There are few examples of working FET devices in which a CP or a MOF is the semiconductor (Fig. 27a and b), and only one example of a MOF being used as a dielectric (Fig. 27c). ${ }^{424}$ For the first case, the first FET-MOF device ever reported was based on the In(III)isophthalate MOF reported by Panda et al. ${ }^{101}$ This MOFs showed conductivity through space with high charge carrier mobility $\left(\mu=4.6 \times 10^{-3} \mathrm{~cm}^{2} \mathrm{~V}^{-1} \mathrm{~s}^{-1}\right.$ at $\left.V_{\mathrm{G}}=-40 \mathrm{~V}\right)$. Long and co-workers prepared single crystal MOF-FETs of chemically reduced $\mathrm{K}_{x} \mathrm{Fe}_{2}(\mathrm{BDP})_{3}(x=0-2)$ and studied the hole and electron mobilities as a function of $x$. The mobilities increased with the progressive reduction until hitting a saturation for $x=1 .^{115}$ The remaining MOF-FETs are based on members of the highly conductive MOG family: Cu-BHT and $\mathrm{Ni}_{3}(\mathrm{HITP})_{2}$. Both showed high charge carrier mobility values: 116 (electrons) and 99 (holes) $\mathrm{cm}^{2} \mathrm{~V}^{-1} \mathrm{~s}^{-1}$ for $\mathrm{Cu}-\mathrm{BHT}^{131}$ and 38-45.4 (hole) $\mathrm{cm}^{2} \mathrm{~V}^{-1} \mathrm{~s}^{-1}$ for $\mathrm{Ni}_{3}(\mathrm{HITP})_{2} \cdot{ }^{425,426}$ Unfortunately, not all MOG-FET devices have performed well. Louie and co-workers prepared FET-type devices of Ni-HBA flakes with both top and bottom contacts that shows a slight current modulation with back gate voltage. ${ }^{141}$ The same small modulation was also observed by Rubio-Giménez et al. in bottom-gated FET devices based on Cu-CAT-1 ultrathin films. ${ }^{156}$

\subsection{Chemical sensors}

Chemical sensors are electronic devices that transduce changes in analyte concentration into electrical signals. MOFs in general have very attractive features that have motivated researchers to use them as active materials in chemical sensors. As it has been described above, they are easily processable as ultrathin films with high surface-to-volume ratios, have intrinsic porosities to host possible analytes and their electronic and structural characteristics are easily tuneable through chemical modification. Hence, the topic of MOFs as sensors has been extensively reviewed in the literature during the past few years. ${ }^{427-431}$ There are various types of MOF sensors depending on the electronic property that is altered with analyte interaction: impedance, chemicapacitive, chemiresistive, Kelvin probe and FET sensors. ${ }^{432}$ The simplest type of sensors are chemiresistors that react to the presence of analytes with changes in their electrical response. Thus, their response can be analysed by monitoring the direct current that flows through the active element at a constant biasing voltage. If the MOF is to act as the active element, chemiresistors require the use of conductive systems. MOGs are thus good candidates for chemiresistive sensors because of their high electrical conductivities comparable to conductive organic polymers and other 2D materials. ${ }^{433}$ The first MOG to be integrated in a chemiresistive device was $\mathrm{Cu}_{3}(\mathrm{HITP})_{2} \cdot{ }^{146}$ Dincă and co-workers fabricated a very simple device by drop casting an acetone suspension of $\mathrm{Cu}_{3}(\mathrm{HITP})_{2}$ onto interdigitated gold electrodes and used it for the reversible chemiresistive sensing of ammonia vapour. Other MOGs chemiresistors soon followed (see Fig. 28), further demonstrating their capability to detect selectively detect small concentrations of various volatile organic compounds (VOCs), ${ }^{434} \mathrm{NH}_{3},{ }^{380,435} \mathrm{NO}, \mathrm{H}_{2} \mathrm{~S}$ and $\mathrm{H}_{2} \mathrm{O},{ }^{436,437}$ methanol ${ }^{157}$ and even anions and cations (potentiometric detection). ${ }^{438}$ However, most research efforts concentrated on sensing performance parameters such as sensitivity, stability and selectivity, whilst the mechanism behind the change in electrical response remained not well understood and host-guest interaction between MOFs and analytes have not been well characterized. ${ }^{128}$ Recently, RubioGiménez et al. proposed a possible origin of this chemiresistive phenomenon in Cu-CAT-1, which lays in the bandgap modification due to the interaction of the $\mathrm{Cu}^{\mathrm{II}}$ ions with gas analytes. ${ }^{158}$

There have also been significant advances to fabricate gas sensor using SCO MOFs. As reviewed beforehand, in porous $\mathrm{Fe}^{\mathrm{II}}$-HCPs the SCO phenomenon is very sensitive to the presence of guest molecules. ${ }^{221,222}$ Therefore, a lot of these frameworks have shown potential as active elements in sensing devices. However only Bousseksou, Salmon and collaborators have fabricated an actual sensing device for VOCs in which the sensing principle is the changes in the SCO transition. ${ }^{439}$ In order to do so, they deposited a patterned thin film of $\mathrm{Fe}(\mathrm{bpac})\left[\mathrm{Pt}(\mathrm{CN})_{4}\right][\mathrm{bpac}=\operatorname{bis}(4$-pyridyl)acetylene $]$ using the previously described LbL technique and photolithography. Changes in the SCO due to the presence of the analytes were then optically detected via changes in the refractive index. The device could detect different aromatic VOCs with a moderate 

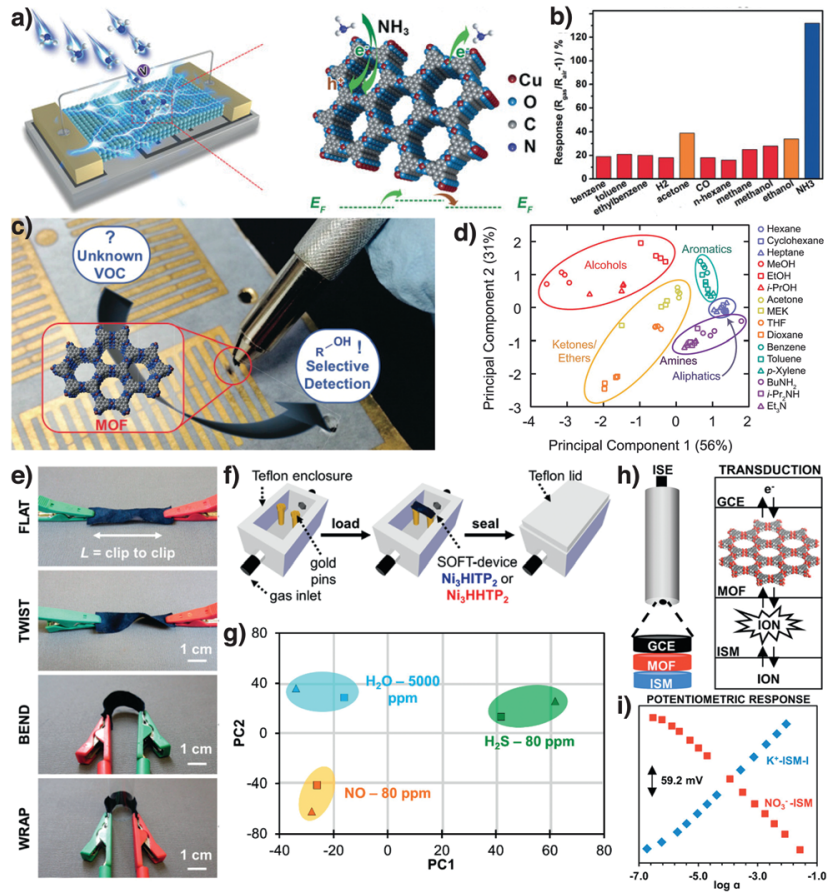

Fig. 28 Examples of MOG chemical sensors. (a) Scheme of a Cu-CAT-1 chemiresistive device and its possible mechanism for the detection of $\mathrm{NH}_{3}$. (b) Column chart showing the selective response of the Cu-CAT-1 device to $\mathrm{NH}_{3}$. (c) Scheme showing the fabrication of a chemiresistive sensor array solvent-free by mechanical "drawing" of MOG powders onto gold electrodes. (d) Principal component analysis of the MOG sensor array responses to various VOCs. (e) Photographs of flexible MOG textile sensing devices. (f) Custom enclosure for dosing MOG textile sensing devices with analytes. (g) Principle component analysis for MOG textile sensing devices showing capability for differentiating $\mathrm{NO}, \mathrm{H}_{2} \mathrm{~S}$, and $\mathrm{H}_{2} \mathrm{O}$. (h) Scheme of the potentiometric ion sensing devices fabricated with the M-CAT-1 MOG family. (i) Potentiometric response of the device to $\mathrm{K}^{+}$and $\mathrm{NO}_{3}{ }^{-}$ions. ( $a$ and $b$ ) Reproduced from ref. 380 with permission from Wiley, copyright 2017. (c and d) Reproduced from ref. 434 with permission from the American Chemical Society, copyright 2015. (e-g) Reproduced from ref. 437 with permission from the American Chemical Society, copyright 2017. ( $h$ and i) Reproduced from ref. 438 with permission from the American Chemical Society, copyright 2018.

sensitivity (few hundreds of ppm). We believe that advances in SCO sensors should progress towards current-based sensing devices. Detection of spin changes via electrical signals has faster dynamics and easier integration with current technology.

\subsection{Switchable devices}

Hitherto, there has been moderate success in developing functional nanodevices based on SCO MOFs and CPs. Apart from the nanostructuration issue discussed above, the SCO properties can be somewhat fragile in a device setting as they might be sensitive to the ambient conditions (e.g. humidity) or to successive switching cycles which might affect reproducibility. Nevertheless, SCO CPs have shown potential in temperature or pressure sensors, memory devices and mechanical actuators.

As detailed in the previous sections, SCO materials show temperature and pressure dependence of their spin state, which translates in measurable changes in their physical properties such as their a)

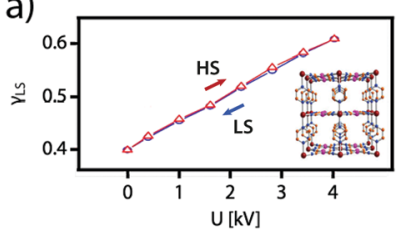

b)
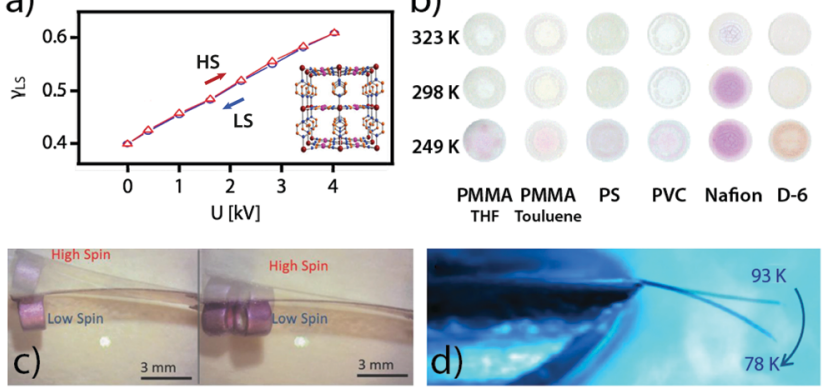

Fig. 29 Examples of SCO switchable devices. (a) Dependance of the LS fraction of $\left[\mathrm{Fe}(\mathrm{pz}) \mathrm{Pt}(\mathrm{CN})_{4}\right]$ with the potential applied to a piezoelement which translates it into pressure, measured at $293 \mathrm{~K}$. (b) Display of various $\left[\mathrm{Fe}\left(\mathrm{NH}_{2}-\mathrm{trz}\right)_{3}\right]\left(\mathrm{BF}_{4}\right) /$ polymer composites that change colour as a function of temperature. (c) A cantilever of [Fe( $(\mathrm{Htrz})_{2}\left(\mathrm{trz}_{\mathrm{rz}}\right]\left(\mathrm{BF}_{4}\right) /$ polymer composite lifting some weight with the SCO-induced actuation. (d) Comparison of a cantilever at 93 and $78 \mathrm{~K}$, highlighting the $\mathrm{SCO}$-induced actuation of $\{\mathrm{Fe}(3-$ $\left.\mathrm{CNpy})\left[\mathrm{Au}(\mathrm{CN})_{2}\right]_{2}\right\} \cdot 2 / 3 \mathrm{H}_{2} \mathrm{O}$. (a) Adapted from ref. 440 with permission from the American Chemical Society, copyright 2019. (b) Adapted from ref. 419 with permission from the Royal Society of Chemistry. (c) Reproduced from ref. 445 with permission from Wiley, copyright 2018. (d) Reproduced from ref. 444 with permission from Springer Nature, copyright 2013.

absorbance spectra or their refractive index. This has been used to fabricate temperature sensors based on photonic displays. For instance, Real, Gaspar, Levchenko and co-workers developed an optical pressure sensor based on the $3 \mathrm{D} \mathrm{Fe}{ }^{\mathrm{II}}-\mathrm{HCP}\left[\mathrm{Fe}(\mathrm{pz}) \mathrm{Pt}(\mathrm{CN})_{4}\right]$, in which small changes in the applied pressure could be correlated to the spin state ratio via an optical absorption band (Fig. 29a). ${ }^{440}$ As visible in Fig. 29b, Lapresta-Fernández et al. used composites of $\left[\mathrm{Fe}\left(\mathrm{NH}_{2}-\mathrm{trz}\right)_{3}\right]\left(\mathrm{BF}_{4}\right)$ and various organic polymers to fabricate thermochromic sensor arrays that macroscopically changed colours between white (HS state), pink, light pink and purple (LS state). ${ }^{419,441}$ As discussed in Section 3.2, SCO CPs have showed potential as electrical memory devices as well. However, a stable readable device has yet to be released, unlike in the case of discrete SCO complexes. ${ }^{42,443}$

SCO CPs have also shown potential as mechanical actuators, as the spin transition also provokes a structural strain that can be translated into mechanical work. Bousseksou and co-workers have pioneered this application with various examples of organic polymer composites with embedded compounds of the 1D CP $\mathrm{Fe}^{\mathrm{II}}$-trz family (Fig. 29c). ${ }^{44-447}$ They also demonstrated this effect on single crystals of a 3D Fe ${ }^{\mathrm{II}}$-HCP (Fig. 29d). ${ }^{444}$ Interestingly, other authors have also showed the powerful effect of the SCO strain of $\left[\mathrm{Fe}(\mathrm{Htrz})_{2}(\mathrm{trz})\right]\left(\mathrm{BF}_{4}\right)^{448}$ and $\left[\mathrm{Fe}(\mathrm{pz}) \mathrm{Pt}(\mathrm{CN})_{4}\right]^{449}$ in the electrical conductivity of organic polymer composites.

\section{Conclusions and future outlook}

The combination in the toolbox provided by coordination chemistry and crystal engineering together with the versatility of molecular frameworks is a fruitful playground for the chemical design of materials that combine properties such as porosity, electrical conductivity and magnetic bistability, all relevant to the development of functional devices. 
The works highlighted above confirm how the design principles required for endowing porous frameworks with electrical conductivity have boosted the development of multiple materials with fine control over charge transport. However, we are still missing a deeper physical understanding of the mechanisms that control it. This limitation is likely imposed by the synthetic difficulties in producing single crystals and the heterogeneity of polycrystalline solids and different methods of measurement, that are typically used. We are confident the next years will witness an increasing number of publications covering the physical mechanisms that control this phenomenon for a clearer understanding of anisotropic transport, charge mobility and carrier density, still required to bridge the gap of this field with organic electronics. Regarding the development of conductive SCO CPs and MOFs, the field is still underdeveloped and holds great potential for further advancement. Current examples are limited to a handful of systems and the through-space and through-bond strategies that have deemed successful for producing non-magnetic conductive MOFs, are not so easy to implement without altering the electrostatic field of the magnetic centres and the SCO properties of the solid. We believe this challenge will be more easily attained by exploring other alternatives that do not involve changes in the metal-organic connectivity and ligand field splitting. Here, combination with conductive polymers or infiltration with redox-active guests might be helpful. Also, the use of mixed-valence systems exemplified by the family of PBAs, constitutes a feasible way to implement higher electrical conductivities compatible with magnetic bistability.

Concerning the processing of these materials, the works reviewed above highlight the broad number of synthetic methods currently available to produce thin films with fine control over their thickness, coverage, roughness, orientation and crystallinity. These are an excellent starting point for their integration into solid-state devices but the effect of nanostructuration over their physical properties relevant to device function is often overlooked, even though conductivity and SCO might be extremely sensitive to particle size reduction effects, pore surface coverage or heterogeneity at the nanoscale. This has not been a major problem for the development of a high number of FETs, sensors, actuators or switching devices that make use exclusively of electrical conductivity or spin transitions. However, the development of applications that exploit the combination of both properties at the atomic level as molecular junctions or spintronic devices are still limited by our poor understanding of nanostructuration or interface effects. We are confident that this area holds great technological potential and we expect an increasing number of fundamental studies deepening into these physical aspects, which are necessary for the integration of these materials into more sophisticated electronic devices.

\section{Conflicts of interest}

There are no conflicts to declare.

\section{Acknowledgements}

The work has been supported by the European Union (ERC Starting Grant Chem-fs-MOF 714122) and the Spanish Ministerio de Ciencia, Innovación y Universidades MICINN (Unit of Excellence María de Maeztu MDM-2015-0538, CTQ2017-83486-P and RTI2018-098568-A-I00). We thank Dr. Garin Escorcia-Ariza, Prof. Rob Ameloot, Prof. José Antonio Real and Prof. Eugenio Coronado for helpful discussions. S. T. thanks the Spanish MINECO for a Ramón y Cajal fellowship (RYC-2016-19817). V. R.-G. acknowledges the Research Foundation Flanders (FWO) for a Marie Skłodowska-Curie Actions - Seal of Excellence Postdoctoral Fellowship (196025/12Z6520N).

\section{Notes and references}

1 Emerging Research Materials, International Roadmap for Devices and Systems, Institute of Electrical and Electronics Engineers, Piscataway, New Jersey, USA, 2017.

2 More Moore, International Roadmap for Devices and Systems, Institute of Electrical and Electronics Engineers, Piscataway, New Jersey, USA, 2017.

3 H. Furukawa, K. E. Cordova, M. O'Keeffe and O. M. Yaghi, Science, 2013, 341, 1230444.

4 W. Lu, Z. Wei, Z.-Y. Gu, T.-F. Liu, J. Park, J. Park, J. Tian, M. Zhang, Q. Zhang, T. Gentle III, M. Bosch and H.-C. Zhou, Chem. Soc. Rev., 2014, 43, 5561-5593.

5 A. Schneemann, V. Bon, I. Schwedler, I. Senkovska, S. Kaskel and R. A. Fischer, Chem. Soc. Rev., 2014, 43, 6062-6096.

6 N. C. Burtch, J. Heinen, T. D. Bennett, D. Dubbeldam and M. D. Allendorf, Adv. Mater., 2018, 30, 1704124.

7 B. F. Hoskins and R. Robson, J. Am. Chem. Soc., 1990, 112, 1546-1554.

8 H. Li, M. Eddaoudi, M. O'Keeffe and O. M. Yaghi, Nature, 1999, 402, 276-279.

9 S. R. Batten, N. R. Champness, X.-M. Chen, J. Garcia-Martinez, S. Kitagawa, L. Öhrström, M. O'Keeffe, M. P. Suh and J. Reedijk, Pure Appl. Chem., 2013, 85, 1715-1724.

10 S. Kitagawa, R. Kitaura and S. Noro, Angew. Chem., Int. Ed., 2004, 43, 2334-2375.

11 M. D. Allendorf, M. E. Foster, F. Léonard, V. Stavila, P. L. Feng, F. P. Doty, K. Leong, E. Y. Ma, S. R. Johnston and A. A. Talin, J. Phys. Chem. Lett., 2015, 6, 1182-1195.

12 J. Liu, P. K. Thallapally, B. P. McGrail, D. R. Brown and J. Liu, Chem. Soc. Rev., 2012, 41, 2308-2322.

13 M. P. Suh, H. J. Park, T. K. Prasad and D.-W. Lim, Chem. Rev., 2012, 112, 782-835.

14 K. Sumida, D. L. Rogow, J. A. Mason, T. M. McDonald, E. D. Bloch, Z. R. Herm, T.-H. Bae and J. R. Long, Chem. Rev., 2012, 112, 724-781.

15 H. Wu, Q. Gong, D. H. Olson and J. Li, Chem. Rev., 2012, 112, 836-868.

16 J. A. Mason, M. Veenstra and J. R. Long, Chem. Sci., 2014, 5, 32-51.

17 Y. He, W. Zhou, G. Qian and B. Chen, Chem. Soc. Rev., 2014, 43, 5657-5678. 
18 J.-R. Li, J. Sculley and H.-C. Zhou, Chem. Rev., 2012, 112, 869-932.

19 X. Zhao, Y. Wang, D.-S. Li, X. Bu and P. Feng, Adv. Mater., 2018, 30, 1705189.

20 J. Lee, O. K. Farha, J. Roberts, K. A. Scheidt, S. T. Nguyen and J. T. Hupp, Chem. Soc. Rev., 2009, 38, 1450-1459.

21 A. Corma, H. García and F. X. Llabrés i Xamena, Chem. Rev., 2010, 110, 4606-4655.

22 M. Yoon, R. Srirambalaji and K. Kim, Chem. Rev., 2012, 112, 1196-1231.

23 P. Falcaro, R. Ricco, C. M. Doherty, K. Liang, A. J. Hill and M. J. Styles, Chem. Soc. Rev., 2014, 43, 5513-5560.

24 V. Stavila, A. A. Talin and M. D. Allendorf, Chem. Soc. Rev., 2014, 43, 5994-6010.

25 I. Stassen, N. Burtch, A. Talin, P. Falcaro, M. Allendorf and R. Ameloot, Chem. Soc. Rev., 2017, 46, 3185-3241.

26 S. K. Bhardwaj, N. Bhardwaj, R. Kaur, J. Mehta, A. L. Sharma, K.-H. Kim and A. Deep, J. Mater. Chem. A, 2018, 6, 14992-15009.

27 M. Tran, K. Kline, Y. Qin, Y. Shen, M. D. Green and S. Tongay, Appl. Phys. Rev., 2019, 6, 041311.

28 J. Wu, J. Chen, C. Wang, Y. Zhou, K. Ba, H. Xu, W. Bao, X. Xu, A. Carlsson, S. Lazar, A. Meingast, Z. Sun and H. Deng, Adv. Sci., 2020, 7, 1903003.

29 G. Molnár, S. Rat, L. Salmon, W. Nicolazzi and A. Bousseksou, Adv. Mater., 2018, 30, 17003862.

$30 \mathrm{~J}$. W. Orton, Semiconductors and the information revolution: magic crystals that made IT happen, Academic Press, Amsterdam, The Netherlands, 2009.

31 G. Wegner, Angew. Chem., Int. Ed. Engl., 1981, 20, 361-381.

32 A. J. Heeger, Angew. Chem., Int. Ed., 2001, 40, 2591-2611.

33 A. G. MacDiarmid, Angew. Chem., Int. Ed., 2001, 40, 2581-2590.

34 V. Saxena and B. D. Malhotra, Curr. Appl. Phys., 2003, 3, 293-305.

35 A. K. Geim and K. S. Novoselov, Nat. Mater., 2007, 6, 183-191.

36 J. K. Wassei and R. B. Kaner, Mater. Today, 2010, 13, 52-59.

37 X. Huang, Z. Yin, S. Wu, X. Qi, Q. He, Q. Zhang, Q. Yan, F. Boey and H. Zhang, Small, 2011, 7, 1876-1902.

38 K. S. Novoselov, V. I. Fal'ko, L. Colombo, P. R. Gellert, M. G. Schwab and K. Kim, Nature, 2012, 490, 192-200.

39 A. C. Ferrari, F. Bonaccorso, V. Fal'ko, K. S. Novoselov, S. Roche, P. Bøggild, S. Borini, F. H. L. Koppens, V. Palermo, N. Pugno, J. A. Garrido, R. Sordan, A. Bianco, L. Ballerini, M. Prato, E. Lidorikis, J. Kivioja, C. Marinelli, T. Ryhänen, A. Morpurgo, J. N. Coleman, V. Nicolosi, L. Colombo, A. Fert, M. Garcia-Hernandez, A. Bachtold, G. F. Schneider, F. Guinea, C. Dekker, M. Barbone, Z. Sun, C. Galiotis, A. N. Grigorenko, G. Konstantatos, A. Kis, M. Katsnelson, L. Vandersypen, A. Loiseau, V. Morandi, D. Neumaier, E. Treossi, V. Pellegrini, M. Polini, A. Tredicucci, G. M. Williams, B. Hee Hong, J.-H. Ahn, J. Min Kim, H. Zirath, B. J. van Wees, H. van der Zant, L. Occhipinti, A. Di Matteo, I. A. Kinloch, T. Seyller, E. Quesnel, X. Feng, K. Teo, N. Rupesinghe, P. Hakonen, S. R. T. Neil, Q. Tannock, T. Löfwander and J. Kinaret, Nanoscale, 2015, 7, 4598-4810.
40 T. W. Ebbesen, H. J. Lezec, H. Hiura, J. W. Bennett, H. F. Ghaemi and T. Thio, Nature, 1996, 382, 54-56.

41 Z. Wu, Z. Chen, X. Du, J. M. Logan, J. Sippel, M. Nikolou, K. Kamaras, J. R. Reynolds, D. B. Tanner, A. F. Hebard and A. G. Rinzler, Science, 2004, 305, 1273-1276.

42 C. Biswas and Y. H. Lee, Adv. Funct. Mater., 2011, 21, 3806-3826.

43 S. Park, M. Vosguerichian and Z. Bao, Nanoscale, 2013, 5, 1727-1752.

44 G. Givaja, P. Amo-Ochoa, C. J. Gomez-Garcia and F. Zamora, Chem. Soc. Rev., 2012, 41, 115-147.

45 R. Murase, B. Ding, Q. Gu and D. M. D’Alessandro, Philos. Trans. R. Soc., A, 2019, 377, 20180226.

46 W. Zhang, J. Chu and M. Hu, Chem. - Asian J., 2020, 15, 1202-1213.

47 L. Sun, M. G. Campbell and M. Dincă, Angew. Chem., Int. Ed., 2016, 55, 3566-3579.

48 C. F. Leong, P. M. Usov and D. M. D’Alessandro, MRS Bull., 2016, 41, 858-864.

49 D. D. Medina, A. Mähringer and T. Bein, Isr. J. Chem., 2018, 58, 1089-1101.

50 P. Li and B. Wang, Isr. J. Chem., 2018, 58, 1010-1018.

51 C.-W. Kung, P.-C. Han, C.-H. Chuang and K. C.-W. Wu, APL Mater., 2019, 7, 110902.

52 X. Deng, J.-Y. Hu, J. Luo, W.-M. Liao and J. He, Top. Curr. Chem., 2020, 378, 27.

53 W.-H. Li, W.-H. Deng, G.-E. Wang and G. Xu, EnergyChem, 2020, 2, 100029.

54 L. S. Xie, G. Skorupskii and M. Dincă, Chem. Rev., 2020, DOI: $10.1021 /$ acs.chemrev.9b00766.

55 J. J. Calvo, S. M. Angel and M. C. So, APL Mater., 2020, 8, 050901.

56 J. Rocha, M. Souto, K. Strutyński and M. Melle-Franco, Chem. - Eur. J., 2020, DOI: 10.1002/chem.202001211.

57 M. D. Allendorf, A. Schwartzberg, V. Stavila and A. A. Talin, Chem. - Eur. J., 2011, 17, 11372-11388.

58 M. Usman, S. Mendiratta and K.-L. Lu, Adv. Mater., 2017, 29, 1605071.

59 S. Han, S. C. Warren, S. M. Yoon, C. D. Malliakas, X. Hou, Y. Wei, M. G. Kanatzidis and B. A. Grzybowski, J. Am. Chem. Soc., 2015, 137, 8169-8175.

60 C.-W. Kung, A. E. Platero-Prats, R. J. Drout, J. Kang, T. C. Wang, C. O. Audu, M. C. Hersam, K. W. Chapman, O. K. Farha and J. T. Hupp, ACS Appl. Mater. Interfaces, 2018, 10, 30532-30540.

61 C.-W. Kung, K. Otake, C. T. Buru, S. Goswami, Y. Cui, J. T. Hupp, A. M. Spokoyny and O. K. Farha, J. Am. Chem. Soc., 2018, 140, 3871-3875.

62 B. Dhara, S. S. Nagarkar, J. Kumar, V. Kumar, P. K. Jha, S. K. Ghosh, S. Nair and N. Ballav, J. Phys. Chem. Lett., 2016, 7, 2945-2950.

63 B. Le Ouay, M. Boudot, T. Kitao, T. Yanagida, S. Kitagawa and T. Uemura, J. Am. Chem. Soc., 2016, 138, 10088-10091.

64 T. C. Wang, I. Hod, C. O. Audu, N. A. Vermeulen, S. T. Nguyen, O. K. Farha and J. T. Hupp, ACS Appl. Mater. Interfaces, 2017, 9, 12584-12591. 
65 A. Nuñez-Lopez, M. Galbiati, N. M. Padial, C. R. Ganivet, S. Tatay, E. Pardo, D. Armentano and C. Martí-Gastaldo, Angew. Chem., Int. Ed., 2019, 58, 9179-9183.

66 A. Jadhav, K. Gupta, P. Ninawe and N. Ballav, Angew. Chem., Int. Ed., 2020, 59, 2215-2219.

67 M.-H. Zeng, Q.-X. Wang, Y.-X. Tan, S. Hu, H.-X. Zhao, L.-S. Long and M. Kurmoo, J. Am. Chem. Soc., 2010, 132, 2561-2563.

68 D. Y. Lee, D. V. Shinde, S. J. Yoon, K. N. Cho, W. Lee, N. K. Shrestha and S.-H. Han, J. Phys. Chem. C, 2014, 118, 16328-16334.

69 D. Y. Lee, E.-K. Kim, N. K. Shrestha, D. W. Boukhvalov, J. K. Lee and S.-H. Han, ACS Appl. Mater. Interfaces, 2015, 7, 18501-18507.

70 D. Y. Lee, I. Lim, C. Y. Shin, S. A. Patil, W. Lee, N. K. Shrestha, J. K. Lee and S.-H. Han, J. Mater. Chem. A, 2015, 3, 22669-22676.

71 G.-P. Li, K. Zhang, H.-Y. Zhao, L. Hou and Y.-Y. Wang, ChemPlusChem, 2017, 82, 716-720.

72 L. Pan, G. Liu, W. Shi, J. Shang, W. R. Leow, Y. Liu, Y. Jiang, S. Li, X. Chen and R.-W. Li, Nat. Commun., 2018, 9, 3813.

73 W. Hai-Ying, G. Jing-Yuan, H. Carol, J. Cheng-Qi, W. Yue, C. F. Leong, D. M. D'Alessandro, L. Tao and Z. Jing-Lin, Angew. Chem., Int. Ed., 2017, 56, 5465-5470.

74 C. F. Leong, C.-H. Wang, C. D. Ling and D. M. D’Alessandro, Polyhedron, 2018, 154, 334-342.

75 J. Su, T.-H. Hu, R. Murase, H.-Y. Wang, D. M. D’Alessandro, M. Kurmoo and J.-L. Zuo, Inorg. Chem., 2019, 58, 3698-3706.

76 Z. Hao, G. Yang, X. Song, M. Zhu, X. Meng, S. Zhao, S. Song and H. Zhang, J. Mater. Chem. A, 2014, 2, 237-244.

77 Y.-Q. Hu, M.-Q. Li, Y. Wang, T. Zhang, P.-Q. Liao, Z. Zheng, X.-M. Chen and Y.-Z. Zheng, Chem. - Eur. J., 2017, 23, 8409-8413.

78 X. Zhang, I. da Silva, R. Fazzi, A. M. Sheveleva, X. Han, B. F. Spencer, S. A. Sapchenko, F. Tuna, E. J. L. McInnes, M. Li, S. Yang and M. Schröder, Inorg. Chem., 2019, 58, 14145-14150.

79 Z. Yin, Q.-X. Wang and M.-H. Zeng, J. Am. Chem. Soc., 2012, 134, 4857-4863.

80 A. A. Talin, A. Centrone, A. C. Ford, M. E. Foster, V. Stavila, P. Haney, R. A. Kinney, V. Szalai, F. El Gabaly, H. P. Yoon, F. Léonard and M. D. Allendorf, Science, 2014, 343, 66.

81 T. Neumann, J. Liu, T. Wächter, P. Friederich, F. Symalla, A. Welle, V. Mugnaini, V. Meded, M. Zharnikov, C. Wöll and W. Wenzel, ACS Nano, 2016, 10, 7085-7093.

82 A. Sengupta, S. Datta, C. Su, T. S. Herng, J. Ding, J. J. Vittal and K. P. Loh, ACS Appl. Mater. Interfaces, 2016, 8, 16154-16159.

83 A. Dragasser, O. Shekhah, O. Zybaylo, C. Shen, M. Buck, C. Woll and D. Schlettwein, Chem. Commun., 2012, 48, 663-665.

84 J. Liu, T. Wächter, A. Irmler, P. G. Weidler, H. Gliemann, F. Pauly, V. Mugnaini, M. Zharnikov and C. Wöll, ACS Appl. Mater. Interfaces, 2015, 7, 9824-9830.

85 Z. Guo, D. K. Panda, K. Maity, D. Lindsey, T. G. Parker, T. E. Albrecht-Schmitt, J. L. Barreda-Esparza, P. Xiong, W. Zhou and S. Saha, J. Mater. Chem. C, 2016, 4, 894-899.
86 S. Goswami, D. Ray, K. Otake, C.-W. Kung, S. J. Garibay, T. Islamoglu, A. Atilgan, Y. Cui, C. J. Cramer, O. K. Farha and J. T. Hupp, Chem. Sci., 2018, 9, 4477-4482.

87 M. Souto, J. Calbo, S. Mañas-Valero, A. Walsh and G. Mínguez Espallargas, Beilstein J. Nanotechnol., 2019, 10, 1883-1893.

88 S. Dalgleish and N. Robertson, Dithiolenes Non-Innocent Redox-Act. Ligands, 2010, 254, 1549-1558.

89 C. Rovira, Chem. Rev., 2004, 104, 5289-5318.

90 M. Iyoda, M. Hasegawa and Y. Miyake, Chem. Rev., 2004, 104, 5085-5114.

91 P. Frère and P. J. Skabara, Chem. Soc. Rev., 2005, 34, 69-98.

92 H.-Y. Wang, L. Cui, J.-Z. Xie, C. F. Leong, D. M. D'Alessandro and J.-L. Zuo, Coord. Chem. Rev., 2017, 345, 342-361.

93 C. Jia, D. Zhang, C.-M. Liu, W. Xu, H. Hu and D. Zhu, New J. Chem., 2002, 26, 490-494.

94 Y. Ding, Q. Chen, J.-C. Zhong, M. Munakata, H. Konaka, G.-L. Ning and H.-Z. Wang, Polyhedron, 2008, 27, 1393-1400.

95 T. L. A. Nguyen, R. Demir-Cakan, T. Devic, M. Morcrette, T. Ahnfeldt, P. Auban-Senzier, N. Stock, A.-M. Goncalves, Y. Filinchuk, J.-M. Tarascon and G. Férey, Inorg. Chem., 2010, 49, 7135-7143.

96 T. C. Narayan, T. Miyakai, S. Seki and M. Dincă, J. Am. Chem. Soc., 2012, 134, 12932-12935.

97 S. S. Park, E. R. Hontz, L. Sun, C. H. Hendon, A. Walsh, T. V. Voorhis and M. Dincă, J. Am. Chem. Soc., 2015, 137, 1774-1777.

98 L. S. Xie and M. Dincă, Isr. J. Chem., 2018, 58, 1119-1122. 99 J. Su, S. Yuan, H.-Y. Wang, L. Huang, J.-Y. Ge, E. Joseph, J. Qin, T. Cagin, J.-L. Zuo and H.-C. Zhou, Nat. Commun., 2017, 8, 2008.

100 S. S. Park, C. H. Hendon, A. J. Fielding, A. Walsh, M. O'Keeffe and M. Dincă, J. Am. Chem. Soc., 2017, 139, 3619-3622.

101 T. Panda and R. Banerjee, Proc. Natl. Acad. Sci., India, Sect. A, 2014, 84, 331-336.

102 D. Chen, H. Xing, Z. Su and C. Wang, Chem. Commun., 2016, 52, 2019-2022.

103 P. I. Scheurle, A. Mähringer, A. C. Jakowetz, P. Hosseini, A. F. Richter, G. Wittstock, D. D. Medina and T. Bein, Nanoscale, 2019, 11, 20949-20955.

104 C. Hua, P. W. Doheny, B. Ding, B. Chan, M. Yu, C. J. Kepert and D. M. D'Alessandro, J. Am. Chem. Soc., 2018, 140, 6622-6630.

105 L. Qu, H. Iguchi, S. Takaishi, F. Habib, C. F. Leong, D. M. D'Alessandro, T. Yoshida, H. Abe, E. Nishibori and M. Yamashita, J. Am. Chem. Soc., 2019, 141, 6802-6806.

106 H. C. Wentz, G. Skorupskii, A. B. Bonfim, J. L. Mancuso, C. H. Hendon, E. H. Oriel, G. T. Sazama and M. G. Campbell, Chem. Sci., 2020, 11, 1342-1346.

107 R. Murase, C. F. Leong and D. M. D'Alessandro, Inorg. Chem., 2017, 56, 14373-14382.

108 Y. Takazaki, Z. Yang, M. Ebihara, K. Inoue and T. Kawamura, Chem. Lett., 2003, 32, 120-121.

109 P. Amo-Ochoa, L. Welte, R. González-Prieto, P. J. Sanz Miguel, C. J. Gómez-García, E. Mateo-Martí, S. Delgado, 
J. Gómez-Herrero and F. Zamora, Chem. Commun., 2010, 46, 3262-3264.

110 J. N. Behera, D. M. D’Alessandro, N. Soheilnia and J. R. Long, Chem. Mater., 2009, 21, 1922-1926.

111 W.-J. Li, C. Han, G. Cheng, S.-L. Chou, H.-K. Liu and S.-X. Dou, Small, 2019, 15, 1900470.

112 F. Gándara, F. J. Uribe-Romo, D. K. Britt, H. Furukawa, L. Lei, R. Cheng, X. Duan, M. O'Keeffe and O. M. Yaghi, Chem. - Eur. J., 2012, 18, 10595-10601.

113 J. G. Park, M. L. Aubrey, J. Oktawiec, K. Chakarawet, L. E. Darago, F. Grandjean, G. J. Long and J. R. Long, J. Am. Chem. Soc., 2018, 140, 8526-8534.

114 L. S. Xie, L. Sun, R. Wan, S. S. Park, J. A. DeGayner, C. H. Hendon and M. Dincă, J. Am. Chem. Soc., 2018, 140, 7411-7414.

115 M. L. Aubrey, B. M. Wiers, S. C. Andrews, T. Sakurai, S. E. ReyesLillo, S. M. Hamed, C.-J. Yu, L. E. Darago, J. A. Mason, J.-O. Baeg, F. Grandjean, G. J. Long, S. Seki, J. B. Neaton, P. Yang and J. R. Long, Nat. Mater., 2018, 17, 625-632.

116 S. Goswami, I. Hod, J. D. Duan, C.-W. Kung, M. Rimoldi, C. D. Malliakas, R. H. Palmer, O. K. Farha and J. T. Hupp, J. Am. Chem. Soc., 2019, 141, 17696-17702.

117 S. Huh, S.-J. Kim and Y. Kim, CrystEngComm, 2016, 18, 345-368.

118 S. R. Ahrenholtz, C. C. Epley and A. J. Morris, J. Am. Chem. Soc., 2014, 136, 2464-2472.

119 L. E. Darago, M. L. Aubrey, C. J. Yu, M. I. Gonzalez and J. R. Long, J. Am. Chem. Soc., 2015, 137, 15703-15711.

120 J. A. DeGayner, I.-R. Jeon, L. Sun, M. Dincă and T. D. Harris, J. Am. Chem. Soc., 2017, 139, 4175-4184.

121 A. Bhattacharjee, D. Bhakat, M. Roy and J. Kusz, Phys. B, 2010, 405, 1546-1550.

122 S. Benmansour, A. Abhervé, P. Gómez-Claramunt, C. Vallés-García and C. J. Gómez-García, ACS Appl. Mater. Interfaces, 2017, 9, 26210-26218.

123 S. A. Sahadevan, A. Abhervé, N. Monni, C. Sáenz de Pipaón, J. R. Galán-Mascarós, J. C. Waerenborgh, B. J. C. Vieira, P. Auban-Senzier, S. Pillet, E.-E. Bendeif, P. Alemany, E. Canadell, M. L. Mercuri and N. Avarvari, J. Am. Chem. Soc., 2018, 140, 12611-12621.

124 L. Liu, J. A. DeGayner, L. Sun, D. Z. Zee and T. D. Harris, Chem. Sci., 2019, 10, 4652-4661.

125 K. S. Pedersen, P. Perlepe, M. L. Aubrey, D. N. Woodruff, S. E. Reyes-Lillo, A. Reinholdt, L. Voigt, Z. Li, K. Borup, M. Rouzières, D. Samohvalov, F. Wilhelm, A. Rogalev, J. B. Neaton, J. R. Long and R. Clérac, Nat. Chem., 2018, 10, 1056-1061.

126 K.-H. Low, V. A. L. Roy, S. S.-Y. Chui, S. L.-F. Chan and C.-M. Che, Chem. Commun., 2010, 46, 7328-7330.

127 L. Sun, C. H. Hendon, M. A. Minier, A. Walsh and M. Dincă, J. Am. Chem. Soc., 2015, 137, 6164-6167.

128 M. Ko, L. Mendecki and K. A. Mirica, Chem. Commun., 2018, 54, 7873-7891.

129 D. Sheberla, L. Sun, M. A. Blood-Forsythe, S. Er, C. R. Wade, C. K. Brozek, A. Aspuru-Guzik and M. Dincă, J. Am. Chem. Soc., 2014, 136, 8859-8862.
130 M. Hmadeh, Z. Lu, Z. Liu, F. Gándara, H. Furukawa, S. Wan, V. Augustyn, R. Chang, L. Liao, F. Zhou, E. Perre, V. Ozolins, K. Suenaga, X. Duan, B. Dunn, Y. Yamamto, O. Terasaki and O. M. Yaghi, Chem. Mater., 2012, 24, 3511-3513.

131 X. Huang, P. Sheng, Z. Tu, F. Zhang, J. Wang, H. Geng, Y. Zou, C. Di, Y. Yi, Y. Sun, W. Xu and D. Zhu, Nat. Commun., 2015, 6, 7408.

132 Y. Cui, J. Yan, Z. Chen, J. Zhang, Y. Zou, Y. Sun, W. Xu and D. Zhu, Adv. Sci., 2019, 6, 1802235.

133 X. Huang, H. Li, Z. Tu, L. Liu, X. Wu, J. Chen, Y. Liang, Y. Zou, Y. Yi, J. Sun, W. Xu and D. Zhu, J. Am. Chem. Soc., 2018, 140, 15153-15156.

134 D. L. Turner, T. P. Vaid, P. W. Stephens, K. H. Stone, A. G. DiPasquale and A. L. Rheingold, J. Am. Chem. Soc., 2008, 130, 14-15.

135 T. Kambe, R. Sakamoto, K. Hoshiko, K. Takada, M. Miyachi, J.-H. Ryu, S. Sasaki, J. Kim, K. Nakazato, M. Takata and H. Nishihara, J. Am. Chem. Soc., 2013, 135, 2462-2465.

136 A. J. Clough, J. W. Yoo, M. H. Mecklenburg and S. C. Marinescu, J. Am. Chem. Soc., 2015, 137, 118-121.

137 T. Pal, T. Kambe, T. Kusamoto, M. L. Foo, R. Matsuoka, R. Sakamoto and H. Nishihara, ChemPlusChem, 2015, 80, 1255-1258.

138 T. Pal, S. Doi, H. Maeda, K. Wada, C. M. Tan, N. Fukui, R. Sakamoto, S. Tsuneyuki, S. Sasaki and H. Nishihara, Chem. Sci., 2019, 10, 5218-5225.

139 X. Sun, K.-H. Wu, R. Sakamoto, T. Kusamoto, H. Maeda and H. Nishihara, Chem. Lett., 2017, 46, 1072-1075.

140 X. Sun, K.-H. Wu, R. Sakamoto, T. Kusamoto, H. Maeda, X. Ni, W. Jiang, F. Liu, S. Sasaki, H. Masunaga and H. Nishihara, Chem. Sci., 2017, 8, 8078-8085.

141 N. Lahiri, N. Lotfizadeh, R. Tsuchikawa, V. V. Deshpande and J. Louie, J. Am. Chem. Soc., 2017, 139, 19-22.

142 J.-H. Dou, L. Sun, Y. Ge, W. Li, C. H. Hendon, J. Li, S. Gul, J. Yano, E. A. Stach and M. Dincă, J. Am. Chem. Soc., 2017, 139, 13608-13611.

143 J. Park, M. Lee, D. Feng, Z. Huang, A. C. Hinckley, A. Yakovenko, X. Zou, Y. Cui and Z. Bao, J. Am. Chem. Soc., 2018, 140, 10315-10323.

144 J. Park, A. C. Hinckley, Z. Huang, D. Feng, A. A. Yakovenko, M. Lee, S. Chen, X. Zou and Z. Bao, J. Am. Chem. Soc., 2018, 140, 14533-14537.

145 X. Huang, S. Zhang, L. Liu, L. Yu, G. Chen, W. Xu and D. Zhu, Angew. Chem., Int. Ed., 2018, 57, 146-150.

146 M. G. Campbell, D. Sheberla, S. F. Liu, T. M. Swager and M. Dincă, Angew. Chem., Int. Ed., 2015, 54, 4349-4352.

147 D. Renhao, Z. Zhikun, D. C. Tranca, Z. Jian, C. Naisa, L. Shaohua, Z. Xiaodong, S. Gotthard and F. Xinliang, Chem. - Eur. J., 2017, 23, 2255-2260.

148 Y. Cui, J. Yan, Z. Chen, W. Xing, C. Ye, X. Li, Y. Zou, Y. Sun, C. Liu, W. Xu and D. Zhu, iScience, 2020, 23, 100812.

149 A. J. Clough, J. M. Skelton, C. A. Downes, A. A. de la Rosa, J. W. Yoo, A. Walsh, B. C. Melot and S. C. Marinescu, J. Am. Chem. Soc., 2017, 139, 10863-10867. 
150 R. Dong, M. Pfeffermann, H. Liang, Z. Zheng, X. Zhu, J. Zhang and X. Feng, Angew. Chem., Int. Ed., 2015, 54, 12058-12063.

151 L. Mendecki, M. Ko, X. Zhang, Z. Meng and K. A. Mirica, J. Am. Chem. Soc., 2017, 139, 17229-17232.

152 J. Cui and Z. Xu, Chem. Commun., 2014, 50, 3986-3988.

153 R. W. Day, D. K. Bediako, M. Rezaee, L. R. Parent, G. Skorupskii, M. Q. Arguilla, C. H. Hendon, I. Stassen, N. C. Gianneschi, P. Kim and M. Dincă, ACS Cent. Sci., 2019, 5, 1959-1964.

154 J. Huang, Y. He, M.-S. Yao, J. He, G. Xu, M. Zeller and Z. Xu, J. Mater. Chem. A, 2017, 5, 16139-16143.

155 W.-H. Li, K. Ding, H.-R. Tian, M.-S. Yao, B. Nath, W.-H. Deng, Y. Wang and G. Xu, Adv. Funct. Mater., 2017, 27, 1702067.

156 V. Rubio-Giménez, M. Galbiati, J. Castells-Gil, N. AlmoraBarrios, J. Navarro-Sánchez, G. Escorcia-Ariza, M. Mattera, T. Arnold, J. Rawle, S. Tatay, E. Coronado and C. MartíGastaldo, Adv. Mater., 2018, 30, 1704291.

157 B. Hoppe, K. D. J. Hindricks, D. P. Warwas, H. A. Schulze, A. Mohmeyer, T. J. Pinkvos, S. Zailskas, M. R. Krey, C. Belke, S. König, M. Fröba, R. J. Haug and P. Behrens, CrystEngComm, 2018, 20, 6458-6471.

158 V. Rubio-Giménez, N. Almora-Barrios, G. Escorcia-Ariza, M. Galbiati, M. Sessolo, S. Tatay and C. Martí-Gastaldo, Angew. Chem., Int. Ed., 2018, 57, 15086-15090.

159 G. Skorupskii, B. A. Trump, T. W. Kasel, C. M. Brown, C. H. Hendon and M. Dincă, Nat. Chem., 2020, 12, 131-136.

160 X. Song, X. Wang, Y. Li, C. Zheng, B. Zhang, C. Di, F. Li, C. Jin, W. Mi, L. Chen and W. Hu, Angew. Chem., Int. Ed., 2020, 59, 1118-1123.

161 M.-S. Yao, J.-J. Zheng, A.-Q. Wu, G. Xu, S. S. Nagarkar, G. Zhang, M. Tsujimoto, S. Sakaki, S. Horike, K. Otake and S. Kitagawa, Angew. Chem., Int. Ed., 2020, 59, 172-176.

162 Y. Jiang, I. Oh, S. H. Joo, O. Buyukcakir, X. Chen, S. H. Lee, M. Huang, W. K. Seong, S. K. Kwak, J.-W. Yoo and R. S. Ruoff, J. Am. Chem. Soc., 2019, 141, 16884-16893.

163 M. Ko, A. Aykanat, M. K. Smith and K. A. Mirica, Sensors, 2017, 17, 2192.

164 D. Sheberla, J. C. Bachman, J. S. Elias, C.-J. Sun, Y. ShaoHorn and M. Dinca, Nat. Mater., 2017, 16, 220-224.

165 L.-P. Tang, L.-M. Tang, H. Geng, Y.-P. Yi, Z. Wei, K.-Q. Chen and H.-X. Deng, Appl. Phys. Lett., 2018, 112, 012101.

166 S. Chen, J. Dai and X. C. Zeng, Phys. Chem. Chem. Phys., 2015, 17, 5954-5958.

167 M. E. Foster, K. Sohlberg, M. D. Allendorf and A. A. Talin, J. Phys. Chem. Lett., 2018, 9, 481-486.

168 L. Sun, S. S. Park, D. Sheberla and M. Dincă, J. Am. Chem. Soc., 2016, 138, 14772-14782.

169 T. Kambe, R. Sakamoto, T. Kusamoto, T. Pal, N. Fukui, K. Hoshiko, T. Shimojima, Z. Wang, T. Hirahara, K. Ishizaka, S. Hasegawa, F. Liu and H. Nishihara, J. Am. Chem. Soc., 2014, 136, 14357-14360.

170 M. E. Foster, K. Sohlberg, C. D. Spataru and M. D. Allendorf, J. Phys. Chem. C, 2016, 120, 15001-15008.
171 R. Dong, P. Han, H. Arora, M. Ballabio, M. Karakus, Z. Zhang, C. Shekhar, P. Adler, P. St. Petkov, A. Erbe, S. C. B. Mannsfeld, C. Felser, T. Heine, M. Bonn, X. Feng and E. Cánovas, Nat. Mater., 2018, 17, 1027-1032.

172 H. Arora, R. Dong, T. Venanzi, J. Zscharschuch, H. Schneider, M. Helm, X. Feng, E. Cánovas and A. Erbe, Adv. Mater., 2020, 32, 1907063.

173 R. Dong, M. Pfeffermann, D. Skidin, F. Wang, Y. Fu, A. Narita, M. Tommasini, F. Moresco, G. Cuniberti, R. Berger, K. Müllen and X. Feng, J. Am. Chem. Soc., 2017, 139, 2168-2171.

174 R. Dong, Z. Zhang, D. C. Tranca, S. Zhou, M. Wang, P. Adler, Z. Liao, F. Liu, Y. Sun, W. Shi, Z. Zhang, E. Zschech, S. C. B. Mannsfeld, C. Felser and X. Feng, Nat. Commun., 2018, 9, 2637.

175 Q. Zhao, S.-H. Li, R.-L. Chai, X. Ren and C. Zhang, ACS Appl. Mater. Interfaces, 2020, 12, 7504-7509.

176 J. Liu, Y. Zhou, Z. Xie, Y. Li, Y. Liu, J. Sun, Y. Ma, O. Terasaki and L. Chen, Angew. Chem., Int. Ed., 2020, 59, 1081-1086.

177 H. Jia, Y. Yao, J. Zhao, Y. Gao, Z. Luo and P. Du, J. Mater. Chem. A, 2018, 6, 1188-1195.

178 N. Hisanori, Y. Nobuhiro, Y. Teppei, S. Kanji and K. Nobuo, Chem. - Eur. J., 2018, 24, 1806-1810.

179 C. Yang, R. Dong, M. Wang, P. St. Petkov, Z. Zhang, M. Wang, P. Han, M. Ballabio, S. A. Bräuninger, Z. Liao, J. Zhang, F. Schwotzer, E. Zschech, H.-H. Klauss, E. Cánovas, S. Kaskel, M. Bonn, S. Zhou, T. Heine and X. Feng, Nat. Commun., 2019, 10, 3260.

180 Z. Meng, A. Aykanat and K. A. Mirica, J. Am. Chem. Soc., 2019, 141, 2046-2053.

181 H. Zhong, K. H. Ly, M. Wang, Y. Krupskaya, X. Han, J. Zhang, J. Zhang, V. Kataev, B. Büchner, I. M. Weidinger, S. Kaskel, P. Liu, M. Chen, R. Dong and X. Feng, Angew. Chem., Int. Ed., 2019, 58, 10677-10682.

182 F. Wang, Z. Liu, C. Yang, H. Zhong, G. Nam, P. Zhang, R. Dong, Y. Wu, J. Cho, J. Zhang and X. Feng, Adv. Mater., 2020, 32, 1905361.

183 J. Dong, X. Han, Y. Liu, H. Li and Y. Cui, Angew. Chem., Int. Ed., 2020, DOI: 10.1002/anie.202004796.

184 Z. Meng, R. M. Stolz and K. A. Mirica, J. Am. Chem. Soc., 2019, 141, 11929-11937.

185 M. Wang, M. Ballabio, M. Wang, H.-H. Lin, B. P. Biswal, X. Han, S. Paasch, E. Brunner, P. Liu, M. Chen, M. Bonn, T. Heine, S. Zhou, E. Cánovas, R. Dong and X. Feng, J. Am. Chem. Soc., 2019, 141, 16810-16816.

186 T. Li, W.-D. Zhang, Y. Liu, Y. Li, C. Cheng, H. Zhu, X. Yan, Z. Li and Z.-G. Gu, J. Mater. Chem. A, 2019, 7, 19676-19681.

187 A. Mähringer, A. C. Jakowetz, J. M. Rotter, B. J. Bohn, J. K. Stolarczyk, J. Feldmann, T. Bein and D. D. Medina, ACS Nano, 2019, 13, 6711-6719.

188 X. Du, J. Zhang, H. Wang, Z. Huang, A. Guo, L. Zhao, Y. Niu, X. Li, B. Wu and Y. Liu, Mater. Chem. Front., 2020, 4, 243-251.

189 L. Sun, B. Liao, D. Sheberla, D. Kraemer, J. Zhou, E. A. Stach, D. Zakharov, V. Stavila, A. A. Talin, Y. Ge, 
M. D. Allendorf, G. Chen, F. Léonard and M. Dincă, Joule, 2017, 1, 168-177.

190 D. Maspoch, D. Ruiz-Molina and J. Veciana, J. Mater. Chem., 2004, 14, 2713-2723.

191 M. Kurmoo, Chem. Soc. Rev., 2009, 38, 1353-1379.

192 M. Clemente-Leon, E. Coronado, C. Marti-Gastaldo and F. M. Romero, Chem. Soc. Rev., 2011, 40, 473-497.

193 D.-F. Weng, Z.-M. Wang and S. Gao, Chem. Soc. Rev., 2011, 40, 3157-3181.

194 E. Coronado and G. Minguez Espallargas, Chem. Soc. Rev., 2013, 42, 1525-1539.

195 T. Grancha, J. Ferrando-Soria, M. Castellano, M. Julve, J. Pasan, D. Armentano and E. Pardo, Chem. Commun., 2014, 50, 7569-7585.

196 K. Liu, X. Zhang, X. Meng, W. Shi, P. Cheng and A. K. Powell, Chem. Soc. Rev., 2016, 45, 2423-2439.

197 G. Mínguez Espallargas and E. Coronado, Chem. Soc. Rev., 2018, 47, 533-557.

198 E. Coronado, Nat. Rev. Mater., 2020, 5, 87-104.

199 A. E. Thorarinsdottir and T. D. Harris, Chem. Rev., 2020, DOI: 10.1021 acs.chemrev.9b00666.

200 O. Kahn and C. J. Martinez, Science, 1998, 279, 44-48.

201 J.-F. Létard, P. Guionneau, L. Goux-Capes, P. Gütlich and H. A. Goodwin, Spin Crossover Transit. Met. Compd. III, 2004, 235, 221-249.

202 C.-M. Jureschi, J. Linares, A. Boulmaali, P. Dahoo, A. Rotaru and Y. Garcia, Sensors, 2016, 16, 187.

203 C. Lefter, V. Davesne, L. Salmon, G. Molnár, P. Demont, A. Rotaru and A. Bousseksou, Magnetochemistry, 2016, $2,18$.

204 K. S. Kumar and M. Ruben, Coord. Chem. Rev., 2017, 346, 176-205.

205 Comptes Rendus Chimie, ed. A. Bousseksou, 2018, vol. 21, pp. 1055-1300.

206 Y. Garcia, V. Niel, M. C. Muñoz and J. A. Real, in Spin Crossover in Transition Metal Compounds I, ed. P. Gütlich and H. A. Goodwin, Springer Berlin Heidelberg, Berlin, Heidelberg, 2004, vol. 223, pp. 229-257.

207 G. Molnár, M. Mikolasek, K. Ridier, A. Fahs, W. Nicolazzi and A. Bousseksou, Ann. Phys., 2019, 531, 1900076.

208 J. A. Real, A. B. Gaspar and M. C. Munoz, Dalton Trans., 2005, 2062-2079.

209 A. Bousseksou, G. Molnár, L. Salmon and W. Nicolazzi, Chem. Soc. Rev., 2011, 40, 3313-3335.

210 P. Gütlich, A. B. Gaspar and Y. Garcia, Beilstein J. Org. Chem., 2013, 9, 342-391.

211 A. B. Gaspar, G. Molnár, A. Rotaru and H. J. Shepherd, C. R. Chim., 2018, 21, 1095-1120.

212 G. Chastanet, M. Lorenc, R. Bertoni and C. Desplanches, C. R. Chim., 2018, 21, 1075-1094.

213 S. Brooker, Chem. Soc. Rev., 2015, 44, 2880-2892.

214 J. A. Real, A. B. Gaspar, V. Niel and M. C. Muñoz, Coord. Chem. Rev., 2003, 236, 121-141.

215 M. Nihei, T. Shiga, Y. Maeda and H. Oshio, Coord. Chem. Rev., 2007, 251, 2606-2621.

216 K. S. Murray, Eur. J. Inorg. Chem., 2008, 3101-3121.
217 A. B. Gaspar, M. Seredyuk and P. Gütlich, J. Mol. Struct., 2009, 924-926, 9-19.

218 J. Olguín, Coord. Chem. Rev., 2020, 407, 213148.

219 K. A. Hofmann and F. Küspert, Z. Anorg. Allg. Chem., 1897, 15, 204-207.

220 M. C. Muñoz and J. A. Real, Coord. Chem. Rev., 2011, 255, 2068-2093.

221 Z.-P. Ni, J.-L. Liu, M. N. Hoque, W. Liu, J.-Y. Li, Y.-C. Chen and M.-L. Tong, Coord. Chem. Rev., 2017, 335, 28-43.

222 R. Ohtani and S. Hayami, Chem. - Eur. J., 2017, 23, 2236-2248.

223 G. Agustí, R. Ohtani, K. Yoneda, A. B. Gaspar, M. Ohba, J. F. Sánchez-Royo, M. C. Muñoz, S. Kitagawa and J. A. Real, Angew. Chem., Int. Ed., 2009, 48, 8944-8947.

224 P. D. Southon, L. Liu, E. A. Fellows, D. J. Price, G. J. Halder, K. W. Chapman, B. Moubaraki, K. S. Murray, J.-F. Létard and C. J. Kepert, J. Am. Chem. Soc., 2009, 131, 10998-11009.

225 C. Bartual-Murgui, N. A. Ortega-Villar, H. J. Shepherd, M. C. Munoz, L. Salmon, G. Molnar, A. Bousseksou and J. A. Real, J. Mater. Chem., 2011, 21, 7217-7222.

226 C. Bartual-Murgui, A. Akou, L. Salmon, G. Molnár, C. Thibault, J. A. Real and A. Bousseksou, Small, 2011, 7, 3385-3391.

227 C. Bartual-Murgui, L. Salmon, A. Akou, N. A. Ortega-Villar, H. J. Shepherd, M. C. Muñoz, G. Molnár, J. A. Real and A. Bousseksou, Chem. - Eur. J., 2012, 18, 507-516.

228 F. J. Muñoz-Lara, A. B. Gaspar, M. C. Muñoz, M. Arai, S. Kitagawa, M. Ohba and J. A. Real, Chem. - Eur. J., 2012, 18, 8013-8018.

229 F. J. Muñoz Lara, A. B. Gaspar, D. Aravena, E. Ruiz, M. C. Muñoz, M. Ohba, R. Ohtani, S. Kitagawa and J. A. Real, Chem. Commun., 2012, 48, 4686-4688.

230 F. J. Muñoz-Lara, A. B. Gaspar, M. C. Muñoz, V. Ksenofontov and J. A. Real, Inorg. Chem., 2013, 52, 3-5.

231 X. Bao, H. J. Shepherd, L. Salmon, G. Molnár, M.-L. Tong and A. Bousseksou, Angew. Chem., Int. Ed., 2013, 52, 1198-1202.

232 L. Pineiro-Lopez, M. Seredyuk, M. C. Munoz and J. A. Real, Chem. Commun., 2014, 50, 1833-1835.

233 L. Piñeiro-López, F. J. Valverde-Muñoz, M. Seredyuk, M. C. Muñoz, M. Haukka and J. A. Real, Inorg. Chem., 2017, 56, 7038-7047.

234 C. D. Polyzou, N. Lalioti, V. Psycharis and V. Tangoulis, New J. Chem., 2017, 41, 12384-12387.

235 F.-L. Liu, D. Li, L.-J. Su and J. Tao, Dalton Trans., 2018, 47, 1407-1411.

236 M. Ohba, K. Yoneda, G. Agustí, M. C. Muñoz, A. B. Gaspar, J. A. Real, M. Yamasaki, H. Ando, Y. Nakao, S. Sakaki and S. Kitagawa, Angew. Chem., Int. Ed., 2009, 48, 4767-4771.

237 D. Aravena, Z. A. Castillo, M. C. Muñoz, A. B. Gaspar, K. Yoneda, R. Ohtani, A. Mishima, S. Kitagawa, M. Ohba, J. A. Real and E. Ruiz, Chem. - Eur. J., 2014, 20, 12864-12873.

238 A. Mishima, T. Koshiyama, J. A. Real and M. Ohba, J. Mater. Chem. C, 2017, 5, 3706-3713.

239 H. Ando, Y. Nakao, H. Sato, M. Ohba, S. Kitagawa and S. Sakaki, Chem. Phys. Lett., 2011, 511, 399-404.

240 M. M. Deshmukh, M. Ohba, S. Kitagawa and S. Sakaki, J. Am. Chem. Soc., 2013, 135, 4840-4849. 
241 M. J. Murphy, K. A. Zenere, F. Ragon, P. D. Southon, C. J. Kepert and S. M. Neville, J. Am. Chem. Soc., 2017, 139, 1330-1335.

242 N. F. Sciortino, F. Ragon, Y. M. Klein, C. E. Housecroft, C. G. Davies, G. N. L. Jameson, G. Chastanet and S. M. Neville, Inorg. Chem., 2018, 57, 11068-11076.

243 K. A. Zenere, S. G. Duyker, E. Trzop, E. Collet, B. Chan, P. W. Doheny, C. J. Kepert and S. M. Neville, Chem. Sci., 2018, 9, 5623-5629.

244 S. Sakaida, K. Otsubo, O. Sakata, C. Song, A. Fujiwara, M. Takata and H. Kitagawa, Nat. Chem., 2016, 8, 377-383.

245 T. Haraguchi, K. Otsubo and H. Kitagawa, Eur. J. Inorg. Chem., 2018, 1697-1706.

246 V. Rubio-Giménez, G. Escorcia-Ariza, C. Bartual-Murgui, C. Sternemann, M. Galbiati, J. Castells-Gil, J. Real, S. Tatay and C. Martí-Gastaldo, Chem. Mater., 2019, 31, 7277-7287.

247 V. Martínez, A. B. Gaspar, M. C. Muñoz, R. Ballesteros, N. Ortega-Villar, V. M. Ugalde-Saldívar, R. Moreno-Esparza and J. A. Real, Eur. J. Inorg. Chem., 2009, 303-310.

248 W. Xue, B.-Y. Wang, J. Zhu, W.-X. Zhang, Y.-B. Zhang, H.-X. Zhao and X.-M. Chen, Chem. Commun., 2011, 47, 10233-10235.

249 B. Djukic and M. T. Lemaire, Inorg. Chem., 2009, 48, 10489-10491.

250 L. Poggini, M. Gonidec, J. H. González-Estefan, G. Pecastaings, B. Gobaut and P. Rosa, Adv. Electron. Mater., 2018, 4, 1800204.

251 L. Poggini, M. Gonidec, R. K. Canjeevaram Balasubramanyam, L. Squillantini, G. Pecastaings, A. Caneschi and P. Rosa, J. Mater. Chem. C, 2019, 7, 5343-5347.

252 J. G. Haasnoot, Coord. Chem. Rev., 2000, 200-202, 131-185.

253 A. Grosjean, P. Négrier, P. Bordet, C. Etrillard, D. Mondieig, S. Pechev, E. Lebraud, J.-F. Létard and P. Guionneau, Eur. J. Inorg. Chem., 2013, 796-802.

254 E. Coronado, J. R. Galán-Mascarós, M. Monrabal-Capilla, J. García-Martínez and P. Pardo-Ibáñez, Adv. Mater., 2007, 19, 1359-1361.

255 C. Lefter, I. A. Gural'skiy, H. Peng, G. Molnár, L. Salmon, A. Rotaru, A. Bousseksou and P. Demont, Phys. Status Solidi RRL, 2014, 8, 191-193.

256 C. Lefter, S. Tricard, H. Peng, G. Molnár, L. Salmon, P. Demont, A. Rotaru and A. Bousseksou, J. Phys. Chem. C, 2015, 119, 8522-8529.

257 I. Soroceanu, A. Graur, E. Coca, L. Salmon, G. Molnar, P. Demont, A. Bousseksou and A. Rotaru, J. Phys. Chem. Lett., 2019, 10, 7391-7396.

258 A. Rotaru, I. A. Gural'skiy, G. Molnar, L. Salmon, P. Demont and A. Bousseksou, Chem. Commun., 2012, 48, 4163-4165.

259 A. Rotaru, J. Dugay, R. P. Tan, I. A. Guralskiy, L. Salmon, P. Demont, J. Carrey, G. Molnár, M. Respaud and A. Bousseksou, Adv. Mater., 2013, 25, 1745-1749.

260 C. Lefter, R. Tan, J. Dugay, S. Tricard, G. Molnár, L. Salmon, J. Carrey, W. Nicolazzi, A. Rotaru and A. Bousseksou, Chem. Phys. Lett., 2016, 644, 138-141.

261 A. Diaconu, S.-L. Lupu, I. Rusu, I.-M. Risca, L. Salmon, G. Molnár, A. Bousseksou, P. Demont and A. Rotaru, J. Phys. Chem. Lett., 2017, 8, 3147-3151.
262 J. Dugay, M. Giménez-Marqués, T. Kozlova, H. W. Zandbergen, E. Coronado and H. S. J. van der Zant, Adv. Mater., 2015, 27, 1288-1293.

263 A. Holovchenko, J. Dugay, M. Giménez-Marqués, R. TorresCavanillas, E. Coronado and H. S. J. van der Zant, $A d v$. Mater., 2016, 28, 7228-7233.

264 J. Dugay, W. Evers, R. Torres-Cavanillas, M. GiménezMarqués, E. Coronado and H. S. J. Van der Zant, J. Phys. Chem. Lett., 2018, 9, 5672-5678.

265 F. Prins, M. Monrabal-Capilla, E. A. Osorio, E. Coronado and H. S. J. van der Zant, Adv. Mater., 2011, 23, 1545-1549.

266 R. Torres-Cavanillas, R. Sanchis-Gual, J. Dugay, M. CoronadoPuchau, M. Giménez-Marqués and E. Coronado, Adv. Mater., 2019, 1900039.

267 M. S. Alam, M. Stocker, K. Gieb, P. Müller, M. Haryono, K. Student and A. Grohmann, Angew. Chem., Int. Ed., 2010, 49, 1159-1163.

268 E. J. Devid, P. N. Martinho, M. V. Kamalakar, I. Šalitroš, Ú. Prendergast, J.-F. Dayen, V. Meded, T. Lemma, R. González-Prieto, F. Evers, T. E. Keyes, M. Ruben, B. Doudin and S. J. van der Molen, ACS Nano, 2015, 9, 4496-4507.

269 C. Lefter, S. Rat, J. S. Costa, M. D. Manrique-Juárez, C. M. Quintero, L. Salmon, I. Séguy, T. Leichle, L. Nicu, P. Demont, A. Rotaru, G. Molnár and A. Bousseksou, Adv. Mater., 2016, 28, 7508-7514.

270 Y. Zhang, I. Séguy, K. Ridier, V. Shalabaeva, M. PiedrahitaBello, A. Rotaru, L. Salmon, G. Molnár and A. Bousseksou, J. Phys.: Condens. Matter, 2020, 32, 214010.

271 X. Zhang, S. Mu, G. Chastanet, N. Daro, T. Palamarciuc, P. Rosa, J.-F. Létard, J. Liu, G. E. Sterbinsky, D. A. Arena, C. Etrillard, B. Kundys, B. Doudin and P. A. Dowben, J. Phys. Chem. C, 2015, 119, 16293-16302.

272 O. Sato, T. Kawakami, M. Kimura, S. Hishiya, S. Kubo and Y. Einaga, J. Am. Chem. Soc., 2004, 126, 13176-13177.

273 N. Shimamoto, S. Ohkoshi, O. Sato and K. Hashimoto, Inorg. Chem., 2002, 41, 678-684.

274 K. R. Dunbar, C. Achim and M. Shatruk, Spin-Crossover Materials, John Wiley \& Sons, Ltd, 2013, pp. 171-202.

275 D. Aguilà, Y. Prado, E. S. Koumousi, C. Mathonière and R. Clérac, Chem. Soc. Rev., 2016, 45, 203-224.

276 Y.-S. Meng, O. Sato and T. Liu, Angew. Chem., Int. Ed., 2018, 57, 12216-12226.

277 G. Molnár, S. Cobo, T. Mahfoud, E. J. M. Vertelman, P. J. van Koningsbruggen, P. Demont and A. Bousseksou, J. Phys. Chem. C, 2009, 113, 2586-2593.

278 T. Mahfoud, G. Molnár, S. Bonhommeau, S. Cobo, L. Salmon, P. Demont, H. Tokoro, S.-I. Ohkoshi, K. Boukheddaden and A. Bousseksou, J. Am. Chem. Soc., 2009, 131, 15049-15054.

279 N. Hoshino, F. Iijima, G. N. Newton, N. Yoshida, T. Shiga, H. Nojiri, A. Nakao, R. Kumai, Y. Murakami and H. Oshio, Nat. Chem., 2012, 4, 921-926.

280 P. Horcajada, C. Serre, D. Grosso, C. Boissière, S. Perruchas, C. Sanchez and G. Férey, Adv. Mater., 2009, 21, 1931-1935. 
281 A. Demessence, P. Horcajada, C. Serre, C. Boissière, D. Grosso, C. Sanchez and G. Férey, Chem. Commun., 2009, 7149-7151.

282 A. Demessence, C. Boissière, D. Grosso, P. Horcajada, C. Serre, G. Férey, G. J. A. A. Soler-Illia and C. Sanchez, J. Mater. Chem., 2010, 20, 7676-7681.

283 R. Dong, T. Zhang and X. Feng, Chem. Rev., 2018, 118, 6189-6235.

284 H. Maeda, R. Sakamoto and H. Nishihara, Langmuir, 2016, 32, 2527-2538.

285 R. Sakamoto, K. Takada, T. Pal, H. Maeda, T. Kambe and H. Nishihara, Chem. Commun., 2017, 53, 5781-5801.

286 C. Lingyun, W. Tingting and W. Cheng, Chin. J. Chem., 2018, 36, 754-764.

287 D. J. Ashworth and J. A. Foster, J. Mater. Chem. A, 2018, 6, 16292-16307.

288 W. Liu, R. Yin, X. Xu, L. Zhang, W. Shi and X. Cao, Adv. Sci., 2019, 6, 1802373.

289 R. Sakamoto, T. Yagi, K. Hoshiko, S. Kusaka, R. Matsuoka, H. Maeda, Z. Liu, Q. Liu, W.-Y. Wong and H. Nishihara, Angew. Chem., Int. Ed., 2017, 56, 3526-3530.

290 Y.-N. Li, S. Wang, Y. Zhou, X.-J. Bai, G.-S. Song, X.-Y. Zhao, T.-Q. Wang, X. Qi, X.-M. Zhang and Y. Fu, Langmuir, 2017, 33, 1060-1065.

291 X. Bai, D. Chen, L. Li, L. Shao, W. He, H. Chen, Y. Li, X. Zhang, L. Zhang, T. Wang, Y. Fu and W. Qi, ACS Appl. Mater. Interfaces, 2018, 10, 25960-25966.

292 I. Langmuir, J. Am. Chem. Soc., 1917, 39, 1848-1906.

293 R. Makiura, S. Motoyama, Y. Umemura, H. Yamanaka, O. Sakata and H. Kitagawa, Nat. Mater., 2010, 9, 565-571.

294 I. Langmuir and V. J. Schaefer, J. Am. Chem. Soc., 1937, 59, 2400-2414.

295 R. Makiura and H. Kitagawa, Eur. J. Inorg. Chem., 2010, 3715-3724.

296 R. Makiura, K. Tsuchiyama and O. Sakata, CrystEngComm, 2011, 13, 5538-5541.

297 S. Motoyama, R. Makiura, O. Sakata and H. Kitagawa, J. Am. Chem. Soc., 2011, 133, 5640-5643.

298 R. Makiura and O. Konovalov, Dalton Trans., 2013, 42, 15931-15936.

299 R. Makiura and O. Konovalov, Sci. Rep., 2013, 3, 2506.

300 R. Makiura, R. Usui, Y. Sakai, A. Nomoto, A. Ogawa, O. Sakata and A. Fujiwara, ChemPlusChem, 2014, 79, 1352-1360.

301 K. Hoshiko, T. Kambe, R. Sakamoto, K. Takada and H. Nishihara, Chem. Lett., 2014, 43, 252-253.

302 Y. Jiang, G. H. Ryu, S. H. Joo, X. Chen, S. H. Lee, X. Chen, M. Huang, X. Wu, D. Luo, Y. Huang, J. H. Lee, B. Wang, X. Zhang, S. K. Kwak, Z. Lee and R. S. Ruoff, ACS Appl. Mater. Interfaces, 2017, 9, 28107-28116.

303 M. Moradi, L. G. Tulli, J. Nowakowski, M. Baljozovic, T. A. Jung and P. Shahgaldian, Angew. Chem., Int. Ed., 2017, 56, 14395-14399.

304 V. Rubio-Giménez, S. Tatay, F. Volatron, F. J. MartínezCasado, C. Martí-Gastaldo and E. Coronado, J. Am. Chem. Soc., 2016, 138, 2576-2584.
305 M. Tsotsalas, A. Umemura, F. Kim, Y. Sakata, J. Reboul, S. Kitagawa and S. Furukawa, J. Mater. Chem., 2012, 22, 10159-10165.

306 J. Benito, S. Sorribas, I. Lucas, J. Coronas and I. Gascon, ACS Appl. Mater. Interfaces, 2016, 8, 16486-16492.

307 A. Ciesielski and P. Samorì, Chem. Soc. Rev., 2014, 43, 381-398.

308 M. Yi and Z. Shen, J. Mater. Chem. A, 2015, 3, 11700-11715.

309 M. I. Kairi, S. Dayou, N. I. Kairi, S. A. Bakar, B. Vigolo and A. R. Mohamed, J. Mater. Chem. A, 2018, 6, 15010-15026.

310 X. Huang, Z. Zeng and H. Zhang, Chem. Soc. Rev., 2013, 42, 1934-1946.

311 H. Li, J. Wu, Z. Yin and H. Zhang, Acc. Chem. Res., 2014, 47, 1067-1075.

312 J. Yu, Q. Wang, D. O'Hare and L. Sun, Chem. Soc. Rev., 2017, 46, 5950-5974.

313 V. Nicolosi, M. Chhowalla, M. G. Kanatzidis, M. S. Strano and J. N. Coleman, Science, 2013, 340, 1226419.

314 C. Tan, X. Cao, X.-J. Wu, Q. He, J. Yang, X. Zhang, J. Chen, W. Zhao, S. Han, G.-H. Nam, M. Sindoro and H. Zhang, Chem. Rev., 2017, 117, 6225-6331.

315 Z. Meiting, L. Qipeng, M. Qinglang and Z. Hua, Small Methods, 2017, 1, 1600030.

316 Q. Zeng, L. Wang, Y. Huang, S.-L. Zheng, Y. He, J. He, W.M. Liao, G. Xu, M. Zeller and Z. Xu, Chem. Commun., 2020, 56, 3645-3648.

317 B. J. Córdova Wong, D. Xu, S.-S. Bao, L.-M. Zheng and J. Lei, ACS Appl. Mater. Interfaces, 2019, 11, 12986-12992.

318 S. Suárez-García, N. N. Adarsh, G. Molnár, A. Bousseksou, Y. Garcia, M. M. Dîrtu, J. Saiz-Poseu, R. Robles, P. Ordejón and D. Ruiz-Molina, ACS Appl. Nano Mater., 2018, 1, 2662-2668.

319 J. López-Cabrelles, S. Mañas-Valero, I. J. Vitórica-Yrezábal, P. J. Bereciartua, J. A. Rodríguez-Velamazán, J. C. Waerenborgh, B. J. C. Vieira, D. Davidovikj, P. G. Steeneken, H. S. J. van der Zant, G. Mínguez Espallargas and E. Coronado, Nat. Chem., 2018, 10, 1001-1007.

320 A. Gallego, C. Hermosa, O. Castillo, I. Berlanga, C. J. GómezGarcía, E. Mateo-Martí, J. I. Martínez, F. Flores, C. GómezNavarro, J. Gómez-Herrero, S. Delgado and F. Zamora, Adv. Mater., 2013, 25, 2141-2146.

321 B. Garai, A. Mallick, A. Das, R. Mukherjee and R. Banerjee, Chem. - Eur. J., 2018, 23, 7361-7366.

322 C. Kutzscher, A. Gelbert, S. Ehrling, C. Schenk, I. Senkovska and S. Kaskel, Dalton Trans., 2017, 46, 16480-16484.

323 Y. Ding, Y.-P. Chen, X. Zhang, L. Chen, Z. Dong, H.L. Jiang, H. Xu and H.-C. Zhou, J. Am. Chem. Soc., 2017, 139, 9136-9139.

324 M. Arnold, P. Kortunov, D. J. Jones, Y. Nedellec, J. Kärger and J. Caro, Eur. J. Inorg. Chem., 2007, 60-64.

325 D. Zacher, A. Baunemann, S. Hermes and R. A. Fischer, J. Mater. Chem., 2007, 17, 2785-2792.

326 C.-W. Kung, T.-H. Chang, L.-Y. Chou, J. T. Hupp, O. K. Farha and K.-C. Ho, Chem. Commun., 2015, 51, 2414-2417. 
327 A. Huang, H. Bux, F. Steinbach and J. Caro, Angew. Chem., Int. Ed., 2010, 49, 4958-4961.

328 A. Huang, W. Dou and J. Caro, J. Am. Chem. Soc., 2010, 132, 15562-15564.

329 G. Blanita, G. Borodi, M. D. Lazar, A.-R. Biris, L. BarbuTudoran, I. Coldea and D. Lupu, RSC $A d v$., 2016, 6, 25967-25974.

330 Y. Yoo and H.-K. Jeong, Chem. Commun., 2008, 2441-2443.

331 H. Bux, F. Liang, Y. Li, J. Cravillon, M. Wiebcke and J. Caro, J. Am. Chem. Soc., 2009, 131, 16000-16001.

332 O. M. Yaghi, G. Li and H. Li, Chem. Mater., 1997, 9, 1074-1076.

333 A. Schoedel, C. Scherb and T. Bein, Angew. Chem., Int. Ed., 2010, 49, 7225-7228.

334 U. Mueller, H. Puetter, M. Hesse, M. Schubert, H. Wessel, J. Huff and M. Guzmann, US Pat., US7968739B2, 2007.

335 R. Ameloot, L. Stappers, J. Fransaer, L. Alaerts, B. F. Sels and D. E. De Vos, Chem. Mater., 2009, 21, 2580-2582.

336 I. Stassen, M. Styles, G. Grenci, H. V. Gorp, W. Vanderlinden, S. D. Feyter, P. Falcaro, D. D. Vos, P. Vereecken and R. Ameloot, Nat. Mater., 2016, 15, 304-310.

337 X. Ma, P. Kumar, N. Mittal, A. Khlyustova, P. Daoutidis, K. A. Mkhoyan and M. Tsapatsis, Science, 2018, 361, 1008-1011.

338 M. Krishtab, I. Stassen, T. Stassin, A. J. Cruz, O. O. Okudur, S. Armini, C. Wilson, S. De Gendt and R. Ameloot, Nat. Commun., 2019, 10, 3729.

339 A. J. Cruz, I. Stassen, M. Krishtab, K. Marcoen, T. Stassin, S. Rodríguez-Hermida, J. Teyssandier, S. Pletincx, R. Verbeke, V. Rubio-Giménez, S. Tatay, C. MartíGastaldo, J. Meersschaut, P. M. Vereecken, S. De Feyter, T. Hauffman and R. Ameloot, Chem. Mater., 2019, 31, 9462-9471.

340 T. Stassin, S. Rodríguez-Hemida, B. Schrode, A. J. Cruz, F. Carraro, D. Kravchenko, V. Creemers, I. Stassen, T. Hauffman, D. E. De Vos, P. Falcaro, R. Resel and R. Ameloot, Chem. Commun., 2019, 55, 10056-10059.

341 T. Stassin, I. Stassen, N. Wauteraerts, A. J. Cruz, M. Kräuter, A. M. Coclite, D. De Vos and R. Ameloot, Eur. J. Inorg. Chem., 2020, 71-74.

342 T. Stassin, I. Stassen, J. Marreiros, A. J. Cruz, R. Verbeke, M. Tu, H. Reinsch, M. Dickmann, W. Egger, I. F. J. Vankelecom, D. E. De Vos and R. Ameloot, Chem. Mater., 2020, 32, 1784-1793.

343 K. B. Lausund and O. Nilsen, Nat. Commun., 2016, 7, 13578.

344 K. B. Lausund, V. Petrovic and O. Nilsen, Dalton Trans., 2017, 46, 16983-16992.

345 D. D. Medina, J. M. Rotter, Y. Hu, M. Dogru, V. Werner, F. Auras, J. T. Markiewicz, P. Knochel and T. Bein, J. Am. Chem. Soc., 2015, 137, 1016-1019.

346 E. Virmani, J. M. Rotter, A. Mähringer, T. von Zons, A. Godt, T. Bein, S. Wuttke and D. D. Medina, J. Am. Chem. Soc., 2018, 140, 4812-4819.

347 H. Lee, L. J. Kepley, H. G. Hong, S. Akhter and T. E. Mallouk, J. Phys. Chem., 1988, 92, 2597-2601.
348 C. M. Bell, S. W. Keller, V. M. Lynch and T. E. Mallouk, Mater. Chem. Phys., 1993, 35, 225-232.

349 H. C. Yang, K. Aoki, H. G. Hong, D. D. Sackett, M. F. Arendt, S. L. Yau, C. M. Bell and T. E. Mallouk, J. Am. Chem. Soc., 1993, 115, 11855-11862.

350 K. Ariga, Y. Yamauchi, G. Rydzek, Q. Ji, Y. Yonamine, K. C.-W. Wu and J. P. Hill, Chem. Lett., 2014, 43, 36-68.

351 O. Shekhah, H. Wang, D. Zacher, R. A. Fischer and C. Wöll, Angew. Chem., Int. Ed., 2009, 48, 5038-5041.

352 O. Zybaylo, O. Shekhah, H. Wang, M. Tafipolsky, R. Schmid, D. Johannsmann and C. Woll, Phys. Chem. Chem. Phys., 2010, 12, 8093-8098.

353 A. Bétard and R. A. Fischer, Chem. Rev., 2012, 112, 1055-1083.

354 J. Liu and C. Woll, Chem. Soc. Rev., 2017, 46, 5730-5770.

355 H. Gliemann and C. Wöll, Mater. Today, 2012, 15, 110-116.

356 L. Heinke, H. Gliemann, P. Tremouilhac and C. Wöll, The Chemistry of Metal-Organic Frameworks: Synthesis, Characterization, and Applications, Wiley-VCH Verlag GmbH \& Co. KGaA, 2016, pp. 523-550.

357 D. Zacher, O. Shekhah, C. Woll and R. A. Fischer, Chem. Soc. Rev., 2009, 38, 1418-1429.

358 O. Shekhah, J. Liu, R. A. Fischer and Ch. Woll, Chem. Soc. Rev., 2011, 40, 1081-1106.

359 D. Zacher, K. Yusenko, A. Bétard, S. Henke, M. Molon, T. Ladnorg, O. Shekhah, B. Schüpbach, T. de los Arcos, M. Krasnopolski, M. Meilikhov, J. Winter, A. Terfort, C. Wöll and R. A. Fischer, Chem. - Eur. J., 2011, 17, 1448-1455.

360 J.-L. Zhuang, A. Terfort and C. Wöll, Coord. Chem. Rev., 2016, 307, 391-424.

361 D. Zacher, R. Schmid, C. Wöll and R. A. Fischer, Angew. Chem., Int. Ed., 2011, 50, 176-199.

362 Z.-G. Gu and J. Zhang, Coord. Chem. Rev., 2019, 378, 513-532.

363 T. P. Vello, M. Strauss, C. A. R. Costa, C. C. Corrêa and C. C. Bof Bufon, Phys. Chem. Chem. Phys., 2020, 22, 5839-5846.

364 P. Falcaro, K. Okada, T. Hara, K. Ikigaki, Y. Tokudome, A. W. Thornton, A. J. Hill, T. Williams, C. Doonan and M. Takahashi, Nat. Mater., 2016, 16, 342-348.

365 K. Ikigaki, K. Okada, Y. Tokudome, T. Toyao, P. Falcaro, C. J. Doonan and M. Takahashi, Angew. Chem., Int. Ed., 2019, 58, 6886-6890.

366 O. Shekhah, H. Wang, S. Kowarik, F. Schreiber, M. Paulus, M. Tolan, C. Sternemann, F. Evers, D. Zacher, R. A. Fischer and C. Wöll, J. Am. Chem. Soc., 2007, 129, 15118-15119.

367 C. Munuera, O. Shekhah, H. Wang, C. Woll and C. Ocal, Phys. Chem. Chem. Phys., 2008, 10, 7257-7261.

368 Z.-G. Gu, A. Pfriem, S. Hamsch, H. Breitwieser, J. Wohlgemuth, L. Heinke, H. Gliemann and C. Wöll, Microporous Mesoporous Mater., 2015, 211, 82-87.

369 H. K. Arslan, O. Shekhah, D. C. F. Wieland, M. Paulus, C. Sternemann, M. A. Schroer, S. Tiemeyer, M. Tolan, R. A. Fischer and C. Wöll, J. Am. Chem. Soc., 2011, 133, 8158-8161. 
370 Y.-Y. Wang, S.-M. Chen, R. Haldar, C. Wöll, Z.-G. Gu and J. Zhang, Adv. Mater. Interfaces, 2018, 5, 1800985.

371 O. Shekhah, Materials, 2010, 3, 1302-1315.

372 S. Wannapaiboon, M. Tu and R. A. Fischer, Adv. Funct. Mater., 2014, 24, 2696-2705.

373 K. Khaletskaya, S. Turner, M. Tu, S. Wannapaiboon, A. Schneemann, R. Meyer, A. Ludwig, G. Van Tendeloo and R. A. Fischer, Adv. Funct. Mater., 2014, 24, 4804-4811.

374 S. Wannapaiboon, M. Tu, K. Sumida, K. Khaletskaya, S. Furukawa, S. Kitagawa and R. A. Fischer, J. Mater. Chem. A, 2015, 3, 23385-23394.

375 M. Tu, S. Wannapaiboon, K. Khaletskaya and R. A. Fischer, Adv. Funct. Mater., 2015, 25, 4470-4479.

376 W. Guo, M. Zha, Z. Wang, E. Redel, Z. Xu and C. Wöll, ACS Appl. Mater. Interfaces, 2016, 8, 24699-24702.

377 Z. Zhou, M.-X. Li, L. Wang, X. He, T. Chi and Z.-X. Wang, Cryst. Growth Des., 2017, 17, 6719-6724.

378 M. R. Tchalala, P. M. Bhatt, K. N. Chappanda, S. R. Tavares, K. Adil, Y. Belmabkhout, A. Shkurenko, A. Cadiau, N. Heymans, G. De Weireld, G. Maurin, K. N. Salama and M. Eddaoudi, Nat. Commun., 2019, 10, 1328.

379 N. L. Torad, S. Zhang, W. A. Amer, M. M. Ayad, M. Kim, J. Kim, B. Ding, X. Zhang, T. Kimura and Y. Yamauchi, Adv. Mater. Interfaces, 2019, 6, 1900849.

380 M.-S. Yao, X.-J. Lv, Z.-H. Fu, W.-H. Li, W.-H. Deng, G.-D. Wu and G. Xu, Angew. Chem., Int. Ed., 2017, 56, 16510-16514.

381 V. Chernikova, O. Shekhah and M. Eddaoudi, ACS Appl. Mater. Interfaces, 2016, 8, 20459-20464.

382 H. K. Arslan, O. Shekhah, J. Wohlgemuth, M. Franzreb, R. A. Fischer and C. Wöll, Adv. Funct. Mater., 2011, 21, 4228-4231.

383 S. Vozar, Y.-C. Poh, T. Serbowicz, M. Bachner, P. Podsiadlo, M. Qin, E. Verploegen, N. Kotov and A. J. Hart, Rev. Sci. Instrum., 2009, 80, 023903.

384 S. Cobo, G. Molnár, J. A. Real and A. Bousseksou, Angew. Chem., Int. Ed., 2006, 45, 5786-5789.

385 G. Molnár, S. Cobo, J. A. Real, F. Carcenac, E. Daran, C. Vieu and A. Bousseksou, Adv. Mater., 2007, 19, 2163-2167.

386 G. Agustí, S. Cobo, A. B. Gaspar, G. Molnár, N. O. Moussa, P. Á. Szilágyi, V. Pálfi, C. Vieu, M. Carmen Muñoz, J. A. Real and A. Bousseksou, Chem. Mater., 2008, 20, 6721-6732.

387 A. Akou, C. Bartual-Murgui, K. Abdul-Kader, M. Lopes, G. Molnár, C. Thibault, C. Vieu, L. Salmon and A. Bousseksou, Dalton Trans., 2013, 42, 16021-16028.

388 K. Otsubo, T. Haraguchi, O. Sakata, A. Fujiwara and H. Kitagawa, J. Am. Chem. Soc., 2012, 134, 9605-9608.

389 T. Haraguchi, K. Otsubo, O. Sakata, A. Fujiwara and H. Kitagawa, Inorg. Chem., 2015, 54, 11593-11595.

390 T. Haraguchi, K. Otsubo, O. Sakata, S. Kawaguchi, A. Fujiwara and H. Kitagawa, Chem. Commun., 2016, 52, 6017-6020.

391 T. Haraguchi, K. Otsubo, O. Sakata, A. Fujiwara and H. Kitagawa, J. Am. Chem. Soc., 2016, 138, 16787-16793.

392 S. Sakaida, T. Haraguchi, K. Otsubo, O. Sakata, A. Fujiwara and H. Kitagawa, Inorg. Chem., 2017, 56, 7606-7609.
393 V. Rubio-Giménez, C. Bartual-Murgui, M. Galbiati, A. Núñez-López, J. Castells-Gil, B. Quinard, P. Seneor, E. Otero, P. Ohresser, A. Cantarero, E. Coronado, J. A. Real, R. Mattana, S. Tatay and C. Martí-Gastaldo, Chem. Sci., 2019, 10, 4038-4047.

394 I. Stassen, D. De Vos and R. Ameloot, Chem. - Eur. J., 2016, 22, 14452-14460.

395 P. Sundberg and M. Karppinen, Eur. J. Inorg. Chem., 2014, 968-974.

396 J. E. Bratvold, G. Carraro, D. Barreca and O. Nilsen, Appl. Surf. Sci., 2015, 347, 861-867.

397 E. Ahvenniemi and M. Karppinen, Dalton Trans., 2016, 45, 10730-10735.

398 D. J. Hagen, L. Mai, A. Devi, J. Sainio and M. Karppinen, Dalton Trans., 2018, 47, 15791-15800.

399 L. D. Salmi, M. J. Heikkilä, E. Puukilainen, T. Sajavaara, D. Grosso and M. Ritala, Microporous Mesoporous Mater., 2013, 182, 147-154.

400 M. Nisula, J. Linnera, A. J. Karttunen and M. Karppinen, Chem. - Eur. J., 2017, 23, 2988-2992.

401 A. Ghazy, M. Safdar, M. Lastusaari and M. Karppinen, Chem. Commun., 2020, 56, 241-244.

402 A. Tanskanen and M. Karppinen, Sci. Rep., 2018, 8, 8976.

403 A. Khayyami, A. Philip and M. Karppinen, Angew. Chem., Int. Ed., 2019, 58, 13400-13404.

404 J. Heiska, M. Nisula, E.-L. Rautama, A. J. Karttunen and M. Karppinen, Dalton Trans., 2020, 49, 1591-1599.

405 M. D. Manrique-Juárez, S. Rat, L. Salmon, G. Molnár, C. M. Quintero, L. Nicu, H. J. Shepherd and A. Bousseksou, Coord. Chem. Rev., 2016, 308, 395-408.

406 H. J. Shepherd, G. Molnár, W. Nicolazzi, L. Salmon and A. Bousseksou, Eur. J. Inorg. Chem., 2013, 653-661.

407 M. Mikolasek, G. Félix, W. Nicolazzi, G. Molnár, L. Salmon and A. Bousseksou, New J. Chem., 2014, 38, 1834-1839.

408 L. Salmon and L. Catala, C. R. Chim., 2018, 21, 1230-1269.

409 T. Mallah and M. Cavallini, C. R. Chim., 2018, 21, 1270-1286.

410 C. D. Polyzou and V. Tangoulis, J. Coord. Chem., 2019, 72, 389-418.

411 I. Boldog, A. B. Gaspar, V. Martínez, P. Pardo-Ibañez, V. Ksenofontov, A. Bhattacharjee, P. Gütlich and J. A. Real, Angew. Chem., Int. Ed., 2008, 47, 6433-6437.

412 F. Volatron, L. Catala, E. Rivière, A. Gloter, O. Stéphan and T. Mallah, Inorg. Chem., 2008, 47, 6584-6586.

413 V. Martínez, I. Boldog, A. B. Gaspar, V. Ksenofontov, A. Bhattacharjee, P. Gütlich and J. A. Real, Chem. Mater., 2010, 22, 4271-4281.

414 J. Larionova, L. Salmon, Y. Guari, A. Tokarev, K. Molvinger, G. Molnár and A. Bousseksou, Angew. Chem., Int. Ed., 2008, 47, 8236-8240.

415 H. Peng, S. Tricard, G. Félix, G. Molnár, W. Nicolazzi, L. Salmon and A. Bousseksou, Angew. Chem., Int. Ed., 2014, 53, 10894-10898.

416 J. R. Galán-Mascarós, E. Coronado, A. Forment-Aliaga, M. Monrabal-Capilla, E. Pinilla-Cienfuegos and M. Ceolin, Inorg. Chem., 2010, 49, 5706-5714. 
417 M. Giménez-Marqués, M. L. García-Sanz de Larrea and E. Coronado, J. Mater. Chem. C, 2015, 3, 7946-7953.

418 D. Nieto-Castro, F. A. Garcés-Pineda, A. Moneo-Corcuera, B. Pato-Doldan, F. Gispert-Guirado, J. Benet-Buchholz and J. R. Galán-Mascarós, Inorg. Chem., 2020, DOI: 10.1021/ acs.inorgchem.9b03284.

419 A. Lapresta-Fernández, M. P. Cuéllar, J. M. Herrera, A. Salinas-Castillo, M. del, C. Pegalajar, S. Titos-Padilla, E. Colacio and L. F. Capitán-Vallvey, J. Mater. Chem. C, 2014, 2, 7292-7303.

420 Y. Raza, F. Volatron, S. Moldovan, O. Ersen, V. Huc, C. Martini, F. Brisset, A. Gloter, O. Stephan, A. Bousseksou, L. Catala and T. Mallah, Chem. Commun., 2011, 47, 11501-11503.

421 O. Iasco, M.-L. Boillot, A. Bellec, R. Guillot, E. Riviere, S. Mazerat, S. Nowak, D. Morineau, A. Brosseau, F. Miserque, V. Repain and T. Mallah, J. Mater. Chem. C, 2017, 5, 11067-11075.

422 V. Shalabaeva, S. Rat, M. D. Manrique-Juarez, A.-C. Bas, L. Vendier, L. Salmon, G. Molnar and A. Bousseksou, J. Mater. Chem. C, 2017, 5, 4419-4425.

423 M. Ruben and K. S. Kumar, Angew. Chem., Int. Ed., 2020, DOI: 10.1002/anie.201911256.

424 Z.-G. Gu, S.-C. Chen, W.-Q. Fu, Q. Zheng and J. Zhang, ACS Appl. Mater. Interfaces, 2017, 9, 7259-7264.

425 G. Wu, J. Huang, Y. Zang, J. He and G. Xu, J. Am. Chem. Soc., 2016, 139, 1360-1363.

426 B. Wang, Y. Luo, B. Liu and G. Duan, ACS Appl. Mater. Interfaces, 2019, 11, 35935-35940.

427 L. E. Kreno, K. Leong, O. K. Farha, M. Allendorf, R. P. Van Duyne and J. T. Hupp, Chem. Rev., 2012, 112, 1105-1125.

428 D. J. Wales, J. Grand, V. P. Ting, R. D. Burke, K. J. Edler, C. R. Bowen, S. Mintova and A. D. Burrows, Chem. Soc. Rev., 2015, 44, 4290-4321.

429 P. Kumar, A. Deep and K.-H. Kim, TrAC, Trends Anal. Chem., 2015, 73, 39-53.

430 F.-Y. Yi, D. Chen, M.-K. Wu, L. Han and H.-L. Jiang, ChemPlusChem, 2016, 81, 675-690.

431 Y. Li, A.-S. Xiao, B. Zou, H.-X. Zhang, K.-L. Yan and Y. Lin, Polyhedron, 2018, 154, 83-97.

432 M. G. Campbell and M. Dincă, Sensors, 2017, 17, 1108.

433 S.-J. Choi and I.-D. Kim, Electron. Mater. Lett., 2018, 14, 221-260.
434 M. G. Campbell, S. F. Liu, T. M. Swager and M. Dincă, J. Am. Chem. Soc., 2015, 137, 13780-13783.

435 L.-A. Cao, M.-S. Yao, H.-J. Jiang, S. Kitagawa, X.-L. Ye, W.-H. Li and G. Xu, J. Mater. Chem. A, 2020, 8, 9085-9090.

436 M. K. Smith, K. E. Jensen, P. A. Pivak and K. A. Mirica, Chem. Mater., 2016, 28, 5264-5268.

437 M. K. Smith and K. A. Mirica, J. Am. Chem. Soc., 2017, 139, 16759-16767.

438 L. Mendecki and K. A. Mirica, ACS Appl. Mater. Interfaces, 2018, 10, 19248-19257.

439 C. Bartual-Murgui, A. Akou, C. Thibault, G. Molnar, C. Vieu, L. Salmon and A. Bousseksou, J. Mater. Chem. C, 2015, 3, 1277-1285.

440 G. Levchenko, G. Bukin, H. Fylymonov, Q. Li, A. B. Gaspar and J. A. Real, J. Phys. Chem. C, 2019, 123, 5642-5646.

441 M. P. Cuéllar, A. Lapresta-Fernández, J. M. Herrera, A. Salinas-Castillo, M. del, C. Pegalajar, S. Titos-Padilla, E. Colacio and L. F. Capitán-Vallvey, Sens. Actuators, B, 2015, 208, 180-187.

442 T. Miyamachi, M. Gruber, V. Davesne, M. Bowen, S. Boukari, L. Joly, F. Scheurer, G. Rogez, T. K. Yamada, P. Ohresser, E. Beaurepaire and W. Wulfhekel, Nat. Commun., 2012, 3, 938.

443 T. Jasper-Toennies, M. Gruber, S. Karan, H. Jacob, F. Tuczek and R. Berndt, Nano Lett., 2017, 17, 6613-6619.

444 H. J. Shepherd, I. A. Gural'skiy, C. M. Quintero, S. Tricard, L. Salmon, G. Molnár and A. Bousseksou, Nat. Commun., 2013, 4, 2607.

445 M. D. Manrique-Juárez, F. Mathieu, A. Laborde, S. Rat, V. Shalabaeva, P. Demont, O. Thomas, L. Salmon, T. Leichle, L. Nicu, G. Molnár and A. Bousseksou, Adv. Funct. Mater., 2018, 28, 1801970.

446 I. A. Gural'skiy, C. M. Quintero, J. S. Costa, P. Demont, G. Molnár, L. Salmon, H. J. Shepherd and A. Bousseksou, J. Mater. Chem. C, 2014, 2, 2949-2955.

447 S. Rat, M. Piedrahita-Bello, L. Salmon, G. Molnár, P. Demont and A. Bousseksou, Adv. Mater., 2018, 30, 1705275.

448 Y.-C. Chen, Y. Meng, Z.-P. Ni and M.-L. Tong, J. Mater. Chem. C, 2015, 3, 945-949.

449 Y.-S. Koo and J. R. Galán-Mascarós, Adv. Mater., 2014, 26, 6785-6789. 